\title{
Norms for words that rhyme
}

\author{
TERRY M. LIBKUMAN \\ Central Michigan University, Mt. Pleasant, Michigan
}

\begin{abstract}
The use of rhyme in learning/memory and cognitive studies is extensive. However, there are very few normative studies for words that rhyme. The current study rectified this problem by collecting rhyme norms for 477 words from 545 subjects. Groups of subjects were given 40 words in serial order and requested, for each word, to generate as many rhymes as possible within a 30-sec interval. The data include several rhyme measures as well as measures of other word attributes that were taken from other sources. In addition, the rhyme responses to the target words were given along with their Thorndike and Lorge (1944) and Kučera and Francis (1967) normative frequencies. Finally, the data were used to investigate various relationships including the spew hypothesis and the accessibility of rhyme sets.
\end{abstract}

What is a rhyme? The definition of rhyme is based entirely on the properties of sound - especially on the quality of assonance. Wood (1943), in his unabridged rhyming dictionary, defined rhyme as the "repetition of an identical accented vowel sound, as well as of all the consonantal and vowel sounds following; with a difference in the consonantal sounds immediately preceding this accented vowel sound" (p. 61). Therefore, the basis of rhyme is the accented vowel. According to Wood, this is usually the last vowel sound to receive a major accent (e.g., ace, face; spoken, broken), although the rhyming vowel sound may be a minor accent instead (e.g., canopy, eternity; honoring, running).

The use of tasks in human learning/memory and cognition that involve rhyme (also referred to as phonological, phonemic, or acoustic similarity tasks, or clang associations) is pervasive. An examination of the literature from the past 40 years revealed over 150 references, the majority of which appeared within the past 20. (A copy of the complete reference list is available from the author on request.)

Historically, rhyme has been used for mnemonic purposes (see, e.g., Furst, 1948). However, the use of thyme in the attempt to understand human cognitive processes is a recent development. Rhyme has been used in a variety of tasks or paradigms to explore a variety of processes and/or phenomena. Specifically, rhyme has been used in recall tasks (Shaw \& Craik, 1989), recognition tasks (Payne, Neely, \& Burns, 1986), paired associate learning tasks (Nelson, Reed, \& McEvoy, 1977), same-different judgments (Kramer \& Donchin, 1987), the reaction time paradigm (Lupker, 1982), memory load tasks (Dornic \& Fernacus, 1987), the retention of connected discourse (King \& Forrester, 1970), verbal discrimination learning tasks

I thank Hajime Otani, and reviewers Cathy McEvoy and Guy Van Orden, for their helpful comments. I also thank Mel Taylor for his invaluable computer assistance. Requests for reprints should be sent to T. M. Libkuman, Psychology Department, Central Michigan University, Mt. Pleasant, MI 48859 (e-mail: 346v5fu.cmuvm).

-Accepted by previous editor, N. John Castellan, Jr.
(Dickel, 1979), the Sternberg paradigm (Lisiecki, 1978), serial recall tasks (Baddeley \& Salamé, 1986), and dichotic listening tasks (Eling, 1983). The processes and/or phenomena studied have included clustering (Bousfield \& Wicklund, 1969), the tip-of-the- tongue state (Kozlowski, 1977), the generation effect (Rabinowitz \& Craik, 1986), short-term memory (Johnson \& Chamberlin, 1970), precategorical acoustic storage (Crowder, 1971), primary and secondary memory (Baddeley, 1964), encoding-retrieval (Asthana \& Goel, 1987), speech and language (Bishop \& Robson, 1989), reading (Besner \& Davelaar, 1982), the word frequency effect (Dunlap \& Dunlap, 1979), developmental processes (Wiegel-Crump \& Dennis, 1986), individual differences involving dyslexia (Spring \& Perry, 1983), psychopathology (Jung, 1918), poets (Kozlowski, 1977), test anxiety (Mueller, 1978), aging (Shaw \& Craik, 1989), and personality (Eysenck, 1981).

Even though many researchers have used rhyme, only few have been concerned with developing norms for words that rhyme. In fact, Noble (1952) viewed clang associates as unacceptable. Flavel, Draguns, Feinberg, and Budion (1958) included rhymes as responses in their free association norms. Other authors (Fisher \& Craik, 1977; Hunt \& Mitchell, 1978; Nelson \& Brooks, 1974; Nelson \& McEvoy, 1979) have generated norms for words that rhyme; these norms are available, but they have not been published. Both of the two published studies (Camden \& Motley, 1982; Horton, 1983) that have presented norms for words that rhyme are limited, because they were developed in conjunction with semantic norms. As a consequence of this lack of norms for words that rhyme only a few studies (Hagen, 1979; Hunt \& Mitchell, 1978; Nelson, McEvoy \& Friedrich, 1982; Nelson, Wheeler, Borden, \& Brooks, 1974; Parkin, 1981) have investigated in a systematic way the relationships between stimuli that rhyme and various dependent measures. Furthermore, without data on other attributes (e.g., frequency) for the rhyming words, the possibility for confounding exists. 
The major objective of the present research was to remedy this problem by collecting rhyme information on 477 words. The intent was to provide a wide range of rhyming values so that the relationship between words that rhyme and various dependent measures could be studied. Also, other attributes of these rhyme words were provided so that the investigator could remove sources of potential confounding. Finally, some of the rhyming relations among the target words were explored (rhyme sets) as well as relationships between target words and their clang associates.

\section{METHOD}

\section{Subjects}

The subjects were 545 undergraduates (mainly introductory psychology students) enrolled at Central Michigan University. The ratio of females to males was approximately $6: 4$.

\section{Materials}

The words were obtained from two sources. Three-hundred and ninety-six words were selected from Paivio, Yuille, and Madigan's (1968) norms ( $N=926$ nouns) for concreteness $(C)$, imagery $(I)$, and meaningfulness $(m)$. The words were selected on the basis of the criterion that at least 1 out of 3 observers could produce at least 1 word that would rhyme with the listed word. With this criterion, a wide range of values for various word attributes (e.g., imagery and frequency) were selected. Furthermore, the distributions of the selected words for $C, I$, and $m$ were similar to the distributions from which the words were taken - that is, negatively skewed. The means and the variances (in parentheses) for $C, I$, and $m$ were 5.52 (3.16), $5.50(1.59)$, and $6.31(0.97)$, respectively. These means were larger and the variances smaller than the values for the set of 925 words $[C=4.95(3.51), I=4.97(1.93), m=5.89(1.21)]$. In terms of Thorndike-Lorge frequencies $(F)$, the present sample possessed more words with higher frequencies than the original set did. In the present sample, $29 \%$ of the words were A or AA $(>49$ occurrences per million), $32 \%$ were in the $10-49$ per million range, and $39 \%$ were less than 10 per million. In contrast, the respective percentages for the Paivio et al. norms were $16 \%, 35 \%$, and $49 \%$. Finally, the distribution for syllables was positively skewed, with 268 monosyllabic words, 191 disyllables, 15 trisyllables, and 3 four-syllable words.

The remaining words were selected from Nelson's (1979) rhyming norms. Nelson's list contains 101 common nouns. Of these, 13 words were duplicates. Therefore, the entire sample for the present study contained 477 words: 389 from Paivio et al. (1968) and 88 from Nelson.

The 477 words were divided into 11 sets of 40 words each and one set of 37 words. Each set was placed in booklet form. The booklets were constructed by taking standard-sized paper $(8.5 \times 11 \mathrm{in}$.) and cutting it in half vertically. On each half sheet, a single word was typed in capital letters 15 times with double spacing. Underlining was typed next to each presentation for the subject's response. The procedure was then repeated for the remaining words. In the construction of the booklets, an attempt was made to ensure that each booklet contained words that represented the alphabet and that the words did not possess any obvious rhyming relationships. Furthermore, for each booklet, two random word orders were developed. Finally, a cover sheet containing instructions was attached to each booklet.

\section{Procedure}

The subjects were tested in their classrooms in groups that ranged in size from 10 to 40 . Each group was informed that the purpose of the investigation was to collect information concerning words that rhyme.

The subjects were instructed to write as many rhyming words as possible for each target word. The examiner then provided an example of a target word with several rhyming words so that the subjects understood the nature of the task. The subjects were then instructed to turn to the first page. The examiner pronounced the target word and then gave the subjects $30 \mathrm{sec}$ to write their responses. The subjects were then instructed to turn to the next page. This procedure was repeated until the 40 target words were presented. A rest break of 5 min was given after the 20 th item. A stopwatch was used to time the various intervals.

\section{RESULTS AND DISCUSSION}

The number of rhyming words that were generated for each target word were tabulated for each subject. Only rhymes with a major accented vowel sound were included, because minor accented vowel sounds are not perceived as rhymes by most people and are not typically used in the psychological literature. Also, linguistic responses that were nonwords or nonrhymes were excluded. The ordinal position of a response word for a target word was not changed if other response words for the target word were excluded. For example, if the response word located in the first ordinal position was excluded (e.g., a rhyme with a minor accented vowel) the response word in the second ordinal position was not moved to the first position. Finally, misspelled words were corrected. Appendix A provides the mean and standard deviation for each target word. In addition, the Paivio et al. (1968) means for $I, C$, and $m$ and the Thorndike-Lorge general frequency $(F)$ are given when available. Finally, a measure of overlap $(O)$ was provided. Overlap is a measure of the extent to which each subject generated the same words in response to the target word. Overlap was determined by dividing each target mean by its set size (i.e., the absolute number of different words that were generated in response to each target item). A value of one would indicate that all subjects generated the same words, whereas a value that approaches zero would indicate that subjects were not generating the same items.

The distribution of the target means was positively skewed $(\mathrm{Sk}=0.43)$ with a median of 2.45 and mean of $2.64(S D=1.93)$ and somewhat platykurtic (KUR = $-0.83)$. The distribution of the overlap variable was positively skewed $(S k=1.00)$ with a median of 0.25 and a mean of $0.27(S D=.17)$. This distribution was leptokurtic $(\mathrm{KUR}=2.16)$. The characteristics of the $I, C, m$, and $F$ distributions have been given in the Materials section.

Several indices of reliability were computed on the basis of the two random orders of the same words for different subjects' tests in different sessions. Pearson correlations were computed for total set size, overlap, and mean rhyme. All three of the correlations were substantial [total set size, $r(154)=.91, p<.01$; overlap, $r(154)$ $=.84, p<.01$; and mean rhyme, $r(154)=98, p<.01]$. These correlations were based on only 7 of the 12 booklets, because knowledge of the two random orders of 5 of the booklets was inadvertently discarded. 
Table 1

Pearson Correlations Among Word Attributes

\begin{tabular}{|c|c|c|c|c|c|c|c|c|c|c|c|c|c|c|c|c|c|}
\hline \multirow[b]{2}{*}{ Attribute } & \multirow[b]{2}{*}{ Syllable } & \multicolumn{2}{|c|}{ Imagery } & \multicolumn{2}{|c|}{ Concreteness } & \multicolumn{2}{|c|}{ Meaningfulness } & \multicolumn{2}{|c|}{ Frequency } & \multicolumn{2}{|c|}{ Rhyme } & \multicolumn{2}{|c|}{$\begin{array}{c}\text { Total } \\
\text { Set Size }\end{array}$} & \multicolumn{2}{|c|}{$\begin{array}{l}\text { First Response } \\
\text { Set Size }\end{array}$} & \multicolumn{2}{|c|}{ Overlap } \\
\hline & & $r$ & $n$ & $r$ & $n$ & $r$ & $n$ & $r$ & $n$ & $r$ & $n$ & $r$ & $n$ & $r$ & $n$ & $r$ & $n$ \\
\hline Syllable & & $-.21 \dagger$ & 389 & $-.17 \dagger$ & 389 & $-.12 *$ & 389 & $-.12 \dagger$ & 478 & $-.63 \dagger$ & 477 & $-.45 \dagger$ & 477 & $-.54 \dagger$ & 477 & .05 & 439 \\
\hline Imagery & & & & $.85 \dagger$ & 389 & $.67 \dagger$ & 389 & $.12^{*}$ & 389 & $.15 \dagger$ & 389 & .08 & 389 & .09 & 389 & .10 & 352 \\
\hline Concreteness & & & & & & $.54 \dagger$ & 389 & .01 & 389 & $.14 \dagger$ & 389 & .09 & 389 & .09 & 389 & .05 & 352 \\
\hline Meaningfulness & & & & & & & & $.28 \dagger$ & 389 & .10 & 389 & .09 & 389 & .07 & 389 & .00 & 352 \\
\hline Frequency & & & & & & & & & & $.11^{*}$ & 389 & .05 & 477 & .06 & 389 & .04 & 439 \\
\hline Rhyme & & & & & & & & & & & & $.77 \dagger$ & 477 & $.83 \dagger$ & 477 & .05 & 440 \\
\hline Total set size & & & & & & & & & & & & & & $.83+$ & 477 & $-.42 \dagger$ & 440 \\
\hline $\begin{array}{l}\text { First response set size } \\
\text { Overlap }\end{array}$ & & & & & & & & & & & & & & & & $-.36 \dagger$ & 440 \\
\hline
\end{tabular}

Note $-n$, sample size. ${ }^{*} p<.05 . \quad \dagger p<.01$.

What are the relationships among the various word attributes? Table 1 provides some information. Note that the number of syllables a word contains is consistently and negatively related to all of the other word attributes, with the exception of the overlap variable. As expected, the semantic cluster of imagery, concreteness, and meaningfulness was highly interrelated (Paivio et al., 1968). Also, the acoustic cluster of rhyme, total set size, first response set size (i.e., the number of different words that were generated as first responses), and overlap was interrelated, with the exception that overlap was only related to the two set size measures. Finally, there were some significant relationships between the acoustic and semantic indices. However, these relationships were not substantial, with none of the correlations exceeding .15 .

Appendix B provides the responses to each target item. This table includes the sample size for each target item as well as the set sizes. Correlations between sample size and set sizes were small $(r s<.05)$ and nonsignificant. The frequency of each response word as a first response and the percentage of subjects who responded with the response word are also given. Finally, the Thorndike and Lorge (1944) and Kučera and Francis (1967) frequencies are given when available.

Is there a relationship between these indices of frequency and the order in which the response words are given? In other words, do subjects "spew" out responses in the order of their frequency (see Underwood \& Schultz, 1960)? The Thorndike-Lorge and Kučera-Francis frequencies were tabulated for each response word by ordinal position. Table 2 indicates that, contrary to the negative relationship produced by the spew hypothesis, there was a tendency for the Kučera and Francis frequencies to increase as ordinal position increased, whereas the Thorndike and Lorge frequencies remained invariant.

Finally, do subjects change their rhyme responses if a different but phonologically similar stimulus is used as the target item? For example, will air produce the same words as chair?

Table 3 contains 89 word rhyme pairs that were a part of the 477 original target nouns. Index of commonality, number of common pairs, and correlations are presented for total set size and first response set size. The index of commonality is a measure of the extent to which each target word in the pair produced the same words. This measure was obtained by dividing the total number of words common to both target words by the total number of words produced by both target words. In most pairs, each target word of a pair was also a response to the other target word in the pair. When this effect occurred, it was treated as if the words were common to both target words and therefore was added to the numerator.

Table 3 also contains Pearson correlations for each rhyme pair. These correlations were based on the number of common pairs. First, the frequency of each response word for each member of the pair was tabulated. (Again note that the target words were treated as a pair.) These frequencies were then converted to percentages. The percentage for each response word was calculated by dividing the response word frequency to a particular target word by the total frequency of response words to the same target. The correlations were then computed on the basis of the percentages.

Total set size means for index of commonality, number of common pairs, and the correlations were .85 $(S D=0.10), 14.93(S D=9.38)$, and $0.74(S D=0.32)$, respectively. Correlations among these measures indicated a positive relationship between the number of common pairs and the Pearson correlations $[r(86)=.24$, $p<.05]$. The relationships between the index of commonality and the Pearson correlations $[r(86)=-.16]$ and the index of commonality and number of common pairs $[r(87)=-.20]$ were not significant $(p>.05)$.

First response set size means for index of commonality, number of common pairs, and the correlations were

Table 2

Thorndike-Lorge (TL) and Kuxera-Francis (KF) Frequencies as a Function of Ordinal Position

\begin{tabular}{crrrrrrr}
\hline \multirow{2}{*}{$\begin{array}{c}\text { Ordinal } \\
\text { Position }\end{array}$} & \multicolumn{3}{c}{$\mathrm{TL}$} & & \multicolumn{3}{c}{$\mathrm{KF}$} \\
\cline { 2 - 4 } \cline { 6 - 8 } & $M$ & $S D$ & $N$ & & $M$ & \multicolumn{1}{c}{$S D$} & $N$ \\
\hline 1 & 30.54 & 19.91 & 14,092 & & 138.96 & 843.08 & 13,824 \\
2 & 29.84 & 20.07 & 11,039 & & 160.16 & $1,264.20$ & 10,845 \\
3 & 29.82 & 20.01 & 8,690 & & 170.57 & $1,235.30$ & 8,519 \\
4 & 30.32 & 19.98 & 6,457 & & 180.84 & $1,363.68$ & 6,349 \\
5 & 30.85 & 19.93 & 4,511 & & 214.04 & $1,190.41$ & 4,376 \\
6 & 31.09 & 20.04 & 2,886 & & 201.18 & 969.75 & 2,766 \\
7 & 31.91 & 19.97 & 1,699 & & 242.58 & $1,158.57$ & 1,618 \\
8 & 30.47 & 19.98 & 922 & & 233.64 & $1,005.93$ & 885 \\
\hline
\end{tabular}


$.71(S D=.16), 5.90(S D=2.05)$, and $.44(S D=.45)$, respectively. Correlations among these measures were small (no correlation exceeded .12), negative, and nonsignificant. Although total set size means were considerably larger than first response set size means, correlations for the two measures of index of commonality $[r(87)=.38$, $p<.01]$, number of common pairs $[r(87)=.63, p<.01]$, and the correlations $[r(86)=.43, p<.01]$ were substantial.

Finally, Pearson correlations, based on the normative values of each target word within each pair, indicated positive relationships for overlap $[r(87)=.88, p<.01]$, total set size $[r(89)=.91, p<.01]$, and first response set size $[r(89)=.78, p<.01]$. The correlations for imagery $[r(50)=.28]$, concreteness $[r(50)=.16]$, meaningfulness $[r(51)=.16]$, and frequency $[r(88)=.11]$ were not significant.

Overall, it appears, at least for words that share the last major accented vowel, that the rhyme set that is generated is independent of the rhyme word that is used to access the set. In other words, any word that is a member of a given rhyme set can be used to generate the members of that rhyme set. This conclusion supports the idea that the words within a rhyme set are connected to a higher order rhyme sound and not to any preexisting connection between the words (Nelson, 1981; Nelson et al., 1982; Rubin, 1990). This notion is further supported by the index of commonality data. The index of commonality should approach unity if all rhyme words within a set are generated from a higher order rhyme sound. Index of commonality means for total set size and first response set size were .85 and .71 , respectively. Is there any consistent strategy that is used to produce rhyme words within a set? A frequency-ordered search does not appear plausible, given the failure to find a negative relationship between word frequency and ordinal position (see Table 2). However, at least for the KučeraFrancis frequencies, subjects appear to be using frequency as a cue for producing rhyme words after the first few rhyme responses have been produced. In other words, after other strategies have lost their effectiveness (e.g., an alphabetical search; Rubin, 1990) subjects may switch to a frequency-ordered search.

Table 3

Index of Commonality, Number of Common Pairs, and Correlations for 89 Rhyme Pairs

\begin{tabular}{|c|c|c|c|c|c|c|}
\hline \multirow[b]{2}{*}{ Rhyme Pair } & \multicolumn{3}{|c|}{ Total Set Size } & \multicolumn{3}{|c|}{ First Response Set Size } \\
\hline & $\begin{array}{c}\text { Index of } \\
\text { Commonality }\end{array}$ & $\begin{array}{l}\text { No. of Common } \\
\text { Pairs } \\
\end{array}$ & $r$ & $\begin{array}{c}\text { Index of } \\
\text { Commonality }\end{array}$ & $\begin{array}{c}\text { No. of Common } \\
\text { Pairs }\end{array}$ & $r$ \\
\hline $\begin{array}{l}\text { Abode } \\
\text { Code }\end{array}$ & .63 & 11 & .83 & .57 & 6 & $\overline{.81}$ \\
\hline $\begin{array}{l}\text { Air } \\
\text { Chair }\end{array}$ & .87 & 27 & .89 & .73 & 8 & .05 \\
\hline $\begin{array}{l}\text { Arm } \\
\text { Charm }\end{array}$ & .89 & 4 & .80 & 1.00 & 4 & .28 \\
\hline $\begin{array}{l}\text { Arm } \\
\text { Farm }\end{array}$ & 1.00 & 4 & .19 & 1.00 & 4 & .48 \\
\hline $\begin{array}{l}\text { Charm } \\
\text { Farm }\end{array}$ & .89 & 4 & .12 & 1.00 & 4 & .22 \\
\hline $\begin{array}{l}\text { Beast } \\
\text { Feast }\end{array}$ & .85 & 11 & .94 & .73 & 4 & .43 \\
\hline $\begin{array}{l}\text { Beat } \\
\text { Meat }\end{array}$ & .81 & 24 & .90 & .67 & 8 & .35 \\
\hline $\begin{array}{l}\text { Beat } \\
\text { Seat }\end{array}$ & .82 & 25 & .89 & .54 & 7 & .35 \\
\hline $\begin{array}{l}\text { Beat } \\
\text { Wheat }\end{array}$ & .85 & 28 & .87 & .69 & 9 & -.10 \\
\hline $\begin{array}{l}\text { Meat } \\
\text { Seat }\end{array}$ & .82 & 22 & .93 & .58 & 7 & .86 \\
\hline $\begin{array}{l}\text { Meat } \\
\text { Wheat }\end{array}$ & .85 & 25 & .78 & .67 & 8 & .50 \\
\hline $\begin{array}{l}\text { Seat } \\
\text { Wheat }\end{array}$ & .82 & 25 & .77 & .69 & 9 & .87 \\
\hline $\begin{array}{l}\text { Belief } \\
\text { Chief }\end{array}$ & .89 & 8 & -.12 & .62 & 4 & -.28 \\
\hline $\begin{array}{l}\text { Belief } \\
\text { Grief }\end{array}$ & .90 & 9 & -.18 & .71 & 5 & -.88 \\
\hline $\begin{array}{l}\text { Belief } \\
\text { Thief }\end{array}$ & .84 & 8 & -.37 & .57 & 4 & .80 \\
\hline $\begin{array}{l}\text { Chief } \\
\text { Grief }\end{array}$ & .89 & 8 & .89 & .93 & 7 & -.56 \\
\hline $\begin{array}{l}\text { Chief } \\
\text { Thief }\end{array}$ & 94 & 8 & .77 & .93 & 7 & .38 \\
\hline
\end{tabular}


Table 3 (Continued)

\begin{tabular}{|c|c|c|c|c|c|c|}
\hline \multirow[b]{2}{*}{ Rhyme Pair } & \multicolumn{3}{|c|}{ Total Set Size } & \multicolumn{3}{|c|}{ First Response Set Size } \\
\hline & $\begin{array}{c}\text { Index of } \\
\text { Commonality }\end{array}$ & $\begin{array}{l}\text { No. of Common } \\
\text { Pairs }\end{array}$ & $r$ & $\begin{array}{c}\text { Index of } \\
\text { Commonality }\end{array}$ & $\begin{array}{c}\text { No. of Common } \\
\text { Pairs } \\
\end{array}$ & $r$ \\
\hline $\begin{array}{l}\text { Grief } \\
\text { Thief }\end{array}$ & .84 & 8 & .76 & .88 & 7 & -.20 \\
\hline $\begin{array}{l}\text { Blood } \\
\text { Flood }\end{array}$ & .93 & 7 & .90 & .80 & 4 & .80 \\
\hline $\begin{array}{l}\text { Boss } \\
\text { Moss }\end{array}$ & .94 & 8 & .96 & .54 & 3 & -.74 \\
\hline $\begin{array}{l}\text { Brain } \\
\text { Plain }\end{array}$ & .68 & 29 & .85 & .56 & 9 & .51 \\
\hline $\begin{array}{l}\text { Brain } \\
\text { Slain }\end{array}$ & .76 & 32 & .77 & .50 & 7 & -.13 \\
\hline $\begin{array}{l}\text { Plain } \\
\text { Slain }\end{array}$ & .74 & 28 & .66 & .56 & 9 & -.02 \\
\hline $\begin{array}{l}\text { Card } \\
\text { Hard }\end{array}$ & .89 & 12 & .99 & .67 & 4 & .50 \\
\hline $\begin{array}{l}\text { Cart } \\
\text { Chart }\end{array}$ & .91 & 10 & -.08 & .90 & 9 & .60 \\
\hline $\begin{array}{l}\text { Cash } \\
\text { Trash }\end{array}$ & .92 & 18 & .87 & .57 & 6 & .98 \\
\hline $\begin{array}{l}\text { Cattle } \\
\text { Rattle }\end{array}$ & .89 & 8 & .95 & .77 & 5 & .85 \\
\hline $\begin{array}{l}\text { Cell } \\
\text { Dell }\end{array}$ & .81 & 13 & .92 & .53 & 4 & .88 \\
\hline $\begin{array}{l}\text { Cell } \\
\text { Smell }\end{array}$ & .79 & 13 & .94 & .71 & 6 & .17 \\
\hline $\begin{array}{l}\text { Dell } \\
\text { Smell }\end{array}$ & .81 & 15 & .81 & .75 & 6 & .45 \\
\hline $\begin{array}{l}\text { Chant } \\
\text { Plant }\end{array}$ & .89 & 8 & .43 & .66 & 4 & -.31 \\
\hline $\begin{array}{l}\text { Church } \\
\text { Search }\end{array}$ & 1.00 & 4 & .46 & 1.00 & 4 & .79 \\
\hline $\begin{array}{l}\text { Clock } \\
\text { Rock }\end{array}$ & .80 & 14 & .85 & .48 & 5 & -.45 \\
\hline $\begin{array}{l}\text { Clock } \\
\text { Shock }\end{array}$ & .88 & 15 & .88 & .78 & 9 & .12 \\
\hline $\begin{array}{l}\text { Rock } \\
\text { Shock }\end{array}$ & .94 & 16 & .85 & .73 & 8 & .29 \\
\hline $\begin{array}{l}\text { Coast } \\
\text { Toast }\end{array}$ & 1.00 & 7 & .89 & .80 & 4 & .64 \\
\hline $\begin{array}{l}\text { Contract } \\
\text { Fact }\end{array}$ & $*$ & * & $*$ & & & \\
\hline $\begin{array}{l}\text { Contract } \\
\text { Pact }\end{array}$ & * & $*$ & * & & & \\
\hline $\begin{array}{l}\text { Fact } \\
\text { Pact }\end{array}$ & 79 & 16 & .87 & .75 & 6 & .74 \\
\hline $\begin{array}{l}\text { Corn } \\
\text { Thorn }\end{array}$ & .71 & 10 & .70 & .59 & 5 & .24 \\
\hline $\begin{array}{l}\text { Crime } \\
\text { Lime }\end{array}$ & 90 & 9 & .77 & .88 & 7 & .10 \\
\hline $\begin{array}{l}\text { Crime } \\
\text { Time }\end{array}$ & .86 & 9 & .72 & .50 & 3 & .73 \\
\hline $\begin{array}{l}\text { Lime } \\
\text { Time }\end{array}$ & .86 & 9 & .89 & .67 & 4 & .31 \\
\hline $\begin{array}{l}\text { Dawn } \\
\text { Lawn }\end{array}$ & .84 & 8 & .98 & .60 & 3 & 1.00 \\
\hline $\begin{array}{l}\text { Deed } \\
\text { Greed }\end{array}$ & .88 & 22 & .83 & .79 & 11 & .62 \\
\hline $\begin{array}{l}\text { Dream } \\
\text { Steam }\end{array}$ & .85 & 14 & .70 & .70 & 8 & .22 \\
\hline $\begin{array}{l}\text { Dream } \\
\text { Stream }\end{array}$ & .84 & 13 & .76 & .82 & 9 & .33 \\
\hline
\end{tabular}


Table 3 (Continued)

\begin{tabular}{|c|c|c|c|c|c|c|}
\hline \multirow[b]{2}{*}{ Rhyme Pair } & \multicolumn{3}{|c|}{ Total Set Size } & \multicolumn{3}{|c|}{ First Response Set Size } \\
\hline & $\begin{array}{c}\text { Index of } \\
\text { Commonality }\end{array}$ & $\begin{array}{c}\text { No. of Common } \\
\text { Pairs }\end{array}$ & $r$ & $\begin{array}{c}\text { Index of } \\
\text { Commonality }\end{array}$ & $\begin{array}{c}\text { No. of Common } \\
\text { Pairs }\end{array}$ & $r$ \\
\hline $\begin{array}{l}\text { Steam } \\
\text { Stream }\end{array}$ & .82 & 14 & .86 & .70 & 7 & .49 \\
\hline $\begin{array}{l}\text { Earth } \\
\text { Mirth }\end{array}$ & .92 & 6 & .93 & .80 & 4 & .95 \\
\hline $\begin{array}{l}\text { Fable } \\
\text { Table }\end{array}$ & 1.00 & 9 & .86 & .86 & 6 & .25 \\
\hline $\begin{array}{l}\text { Fight } \\
\text { Sight }\end{array}$ & .80 & 24 & .94 & .78 & 9 & .57 \\
\hline $\begin{array}{l}\text { Fire } \\
\text { Spire }\end{array}$ & .66 & 20 & .72 & .69 & 9 & .24 \\
\hline $\begin{array}{l}\text { Fire } \\
\text { Tire }\end{array}$ & .62 & 15 & .94 & .78 & 9 & .69 \\
\hline $\begin{array}{l}\text { Spire } \\
\text { Tire }\end{array}$ & .56 & 13 & .98 & .61 & 7 & .98 \\
\hline $\begin{array}{l}\text { Flower } \\
\text { Power }\end{array}$ & .80 & 8 & .93 & .83 & 5 & .76 \\
\hline $\begin{array}{l}\text { Flower } \\
\text { Tower }\end{array}$ & .78 & 9 & .84 & .67 & 4 & 1.00 \\
\hline $\begin{array}{l}\text { Power } \\
\text { Tower }\end{array}$ & .67 & 7 & .80 & .83 & 5 & .77 \\
\hline $\begin{array}{l}\text { Form } \\
\text { Storm }\end{array}$ & 1.00 & 5 & .80 & .89 & 4 & .53 \\
\hline $\begin{array}{l}\text { Gift } \\
\text { Shift }\end{array}$ & .78 & 7 & .85 & .80 & 6 & .88 \\
\hline $\begin{array}{l}\text { Girdle } \\
\text { Hurdle }\end{array}$ & .75 & 3 & .55 & .75 & 3 & 1.00 \\
\hline $\begin{array}{l}\text { Gist } \\
\text { Mist }\end{array}$ & .69 & 10 & .77 & .78 & 7 & .42 \\
\hline $\begin{array}{l}\text { Glove } \\
\text { Love }\end{array}$ & 1.00 & 5 & .62 & 1.00 & 4 & .70 \\
\hline $\begin{array}{l}\text { Glove } \\
\text { Shove }\end{array}$ & 1.00 & 5 & .96 & 1.00 & 4 & .65 \\
\hline $\begin{array}{l}\text { Love } \\
\text { Shove }\end{array}$ & 1.00 & 5 & .35 & 1.00 & 4 & -.05 \\
\hline $\begin{array}{l}\text { Gore } \\
\text { Lore }\end{array}$ & .86 & 31 & .90 & .55 & 8 & .68 \\
\hline $\begin{array}{l}\text { Gore } \\
\text { Shore }\end{array}$ & .93 & 33 & .92 & .40 & 5 & .89 \\
\hline $\begin{array}{l}\text { Gore } \\
\text { Store }\end{array}$ & .92 & 33 & .82 & .52 & 7 & .52 \\
\hline $\begin{array}{l}\text { Lore } \\
\text { Shore }\end{array}$ & .88 & 33 & .77 & .43 & 6 & .93 \\
\hline $\begin{array}{l}\text { Lore } \\
\text { Store }\end{array}$ & .87 & 33 & .74 & .45 & 7 & .97 \\
\hline $\begin{array}{l}\text { Shore } \\
\text { Store }\end{array}$ & .91 & 34 & .80 & .52 & 7 & .62 \\
\hline $\begin{array}{l}\text { Hide } \\
\text { Pride }\end{array}$ & .81 & 26 & .80 & .52 & 6 & .91 \\
\hline $\begin{array}{l}\text { Hoof } \\
\text { Roof }\end{array}$ & .91 & 5 & .71 & .91 & 5 & .44 \\
\hline $\begin{array}{l}\text { Ink } \\
\text { Link }\end{array}$ & .94 & 17 & .85 & .88 & 8 & 42 \\
\hline $\begin{array}{l}\text { Jail } \\
\text { Nail }\end{array}$ & .81 & 27 & .81 & .59 & 8 & 42 \\
\hline $\begin{array}{l}\text { Jail } \\
\text { Pail }\end{array}$ & .85 & 32 & .83 & .53 & 8 & .90 \\
\hline $\begin{array}{l}\text { Nail } \\
\text { Pail }\end{array}$ & .88 & 30 & .84 & .48 & 7 & .21 \\
\hline $\begin{array}{l}\text { Jump } \\
\text { Lump }\end{array}$ & .88 & 15 & .90 & .53 & 4 & .96 \\
\hline
\end{tabular}


APPENDIX A (Continued)

\begin{tabular}{|c|c|c|c|c|c|c|c|c|c|c|c|c|c|c|c|}
\hline \multirow[b]{2}{*}{ Target Words } & \multicolumn{2}{|c|}{$R$} & \multirow[b]{2}{*}{$I$} & \multirow[b]{2}{*}{$C$} & \multirow[b]{2}{*}{$m$} & \multirow[b]{2}{*}{$F^{a}$} & & & & & & & & & \\
\hline & $M$ & $S D$ & & & & & $O$ & Target Words & $M$ & $S D$ & $I$ & $C$ & $m$ & $F^{\text {a }}$ & $O$ \\
\hline Chief & 2.52 & 1.32 & 6.07 & 5.87 & 6.08 & $\mathrm{AA}$ & .32 & Fight & 6.78 & 2.11 & & & & $\mathrm{AA}$ & .21 \\
\hline Child & 2.87 & 1.44 & 6.50 & 6.87 & 7.04 & $\mathrm{AA}$ & .26 & Figment & .72 & .45 & 2.30 & 1.90 & 4.00 & 0 & .72 \\
\hline Chin & 5.32 & 2.24 & 6.43 & 6.96 & 5.28 & 27 & .27 & Fiord & .00 & .00 & 5.70 & 6.69 & 6.48 & 2 & \\
\hline Chloride & 1.52 & 1.40 & 4.20 & 6.28 & 5.75 & 2 & .10 & Fire & 4.41 & 1.79 & 6.70 & 6.66 & 7.36 & $\mathrm{AA}$ & .14 \\
\hline Church & 2.35 & 1.00 & 6.63 & 6.50 & 7.52 & AA & .59 & Flag & 6.00 & 1.65 & 6.60 & 6.94 & 6.54 & A & .29 \\
\hline Cigar & .00 & .00 & 6.80 & 6.96 & 6.22 & 16 & & Flask & 2.27 & 1.09 & 6.50 & 7.00 & 6.28 & 4 & .32 \\
\hline Circle & .00 & .00 & 6.23 & 6.00 & 4.88 & AA & & Flesh & .93 & .76 & 6.13 & 6.90 & 5.84 & A & 47 \\
\hline Circuit & .00 & .00 & 4.97 & 5.63 & 6.38 & 21 & & Flood & 2.84 & 1.22 & 6.33 & 6.62 & 6.36 & A & .36 \\
\hline City & 2.81 & 1.45 & 6.43 & 6.41 & 7.72 & AA & .22 & Flower & 2.55 & 1.59 & 6.57 & 6.96 & 7.13 & $\mathrm{AA}$ & .23 \\
\hline Claw & 3.24 & 1.94 & 6.33 & 6.89 & 6.67 & 23 & .22 & Foam & 3.00 & 1.33 & 6.33 & 6.73 & 6.44 & 21 & .33 \\
\hline Clock & 5.64 & 1.56 & 6.50 & 6.94 & 7.08 & A & .31 & Folly & 3.71 & 1.30 & 2.93 & 2.63 & 4.40 & 29 & .34 \\
\hline Cloth & 1.49 & 1.00 & & & & A & .30 & Forest & .45 & .69 & 6.63 & 6.69 & 9.12 & $\mathrm{AA}$ & .15 \\
\hline Coach & 1.35 & .85 & & & & 42 & .27 & Fork & 2.16 & 1.05 & 6.57 & 6.94 & 7.08 & 31 & .36 \\
\hline Coast & 4.43 & 1.42 & 6.13 & 6.59 & 6.40 & A & .63 & Form & 1.76 & 1.08 & 4.30 & 4.08 & 4.60 & $\mathrm{AA}$ & .35 \\
\hline Coat & 4.84 & 1.50 & & & & $\mathrm{AA}$ & .32 & Fowl & 2.45 & 1.41 & 5.87 & 6.58 & 7.36 & 20 & .20 \\
\hline Code & 3.40 & 1.70 & 4.53 & 4.53 & 6.63 & 21 & .18 & Fox & 3.90 & 1.68 & 6.73 & 7.00 & 7.40 & 25 & .22 \\
\hline Coffee & .81 & .39 & 6.73 & 6.89 & 7.28 & A & .82 & Freedom & .00 & .00 & 3.83 & 1.98 & 6.36 & A & \\
\hline Coin & 1.43 & .92 & 6.50 & 6.90 & 7.09 & A & .24 & Friction & 1.26 & 1.08 & 4.33 & 4.26 & 5.28 & 9 & .16 \\
\hline College & .38 & .49 & 6.20 & 6.38 & 7.28 & $\mathrm{AA}$ & .38 & Friend & 4.76 & 1.76 & 6.37 & 4.89 & 6.40 & $\mathrm{AA}$ & .19 \\
\hline Comrade & .00 & .00 & 5.57 & 5.65 & 5.58 & 26 & & Frog & 4.15 & 1.63 & 6.73 & 6.96 & 6.56 & 25 & .30 \\
\hline Context & .11 & .31 & 2.13 & 2.73 & 4.44 & 1 & .05 & Fun & 5.10 & 1.61 & 5.23 & 2.45 & 5.50 & 20 & .30 \\
\hline Contract & .00 & .00 & 4.50 & 5.24 & 6.32 & $\mathrm{~A}$ & & Fur & 2.70 & 1.33 & 6.23 & 6.69 & 7.36 & A & .27 \\
\hline Corn & 3.49 & 1.42 & 6.47 & 6.90 & 6.96 & A & .35 & Garden & 1.05 & .84 & 6.73 & 6.83 & 6.36 & $\mathrm{AA}$ & .53 \\
\hline Corner & .76 & .86 & 6.13 & 6.65 & 5.67 & $\mathrm{AA}$ & .19 & Geese & 2.54 & 1.33 & 6.53 & 6.83 & 7.48 & 26 & .16 \\
\hline Corpse & .21 & .47 & 6.50 & 6.89 & 6.52 & 9 & .21 & Gem & .82 & .76 & 6.40 & 6.59 & 6.88 & 24 & .14 \\
\hline Cottage & .13 & .34 & 6.50 & 6.90 & 7.76 & 46 & .07 & Gender & 4.76 & 1.40 & 2.90 & 3.63 & 5.41 & 1 & .37 \\
\hline Cotton & 1.06 & .88 & 6.00 & 6.90 & 7.13 & $\mathrm{AA}$ & .35 & Ghost & 4.55 & 1.48 & 5.37 & 2.97 & 6.00 & 32 & .65 \\
\hline Cradle & .92 & .81 & 6.23 & 6.94 & 6.96 & 21 & .31 & Gift & 2.58 & 1.06 & 5.77 & 5.95 & 7.04 & A & .37 \\
\hline Crag & 6.04 & 2.03 & 4.77 & 6.42 & 5.44 & 8 & .32 & Gilt & 3.87 & 1.60 & 4.20 & 5.14 & 5.32 & 5 & .35 \\
\hline Crime & 3.39 & 1.73 & 4.43 & 3.81 & 6.84 & A & .34 & Girdle & 1.18 & 1.04 & & & & 14 & .39 \\
\hline Cup & 1.63 & .98 & & & & $\mathrm{AA}$ & .41 & Girl & 2.70 & 1.32 & 6.87 & 6.83 & 5.12 & $\mathrm{AA}$ & .25 \\
\hline Dance & 2.45 & 1.33 & & & & $\mathrm{AA}$ & .20 & Gist & 3.69 & 1.78 & 1.97 & 1.77 & 3.33 & 1 & .26 \\
\hline Dawn & 2.58 & 1.06 & 6.37 & 5.83 & 7.36 & A & .26 & Glory & 2.12 & 1.18 & 4.13 & 1.77 & 5.88 & A & .30 \\
\hline Death & .74 & .76 & 5.00 & 2.97 & 7.12 & $\mathrm{AA}$ & .25 & Glove & 2.56 & .93 & & & & 43 & .51 \\
\hline Deceit & 1.74 & 1.83 & 3.30 & 1.66 & 4.92 & 8 & .08 & Glutton & 1.53 & .79 & 5.77 & 5.77 & 5.32 & 2 & .76 \\
\hline Deck & 3.02 & 1.30 & & & & A & .28 & Gold & 6.00 & 1.34 & 6.47 & 6.76 & 6.36 & AA & .50 \\
\hline Deed & 5.76 & 2.22 & 3.63 & 4.19 & 5.32 & A & .24 & Golf & .11 & .32 & 6.70 & 6.10 & 8.16 & 26 & .11 \\
\hline Dell & 5.88 & 1.68 & 3.37 & 5.58 & 4.56 & 11 & .33 & Gore & 7.18 & 2.06 & 4.67 & 4.79 & 5.50 & 7 & .21 \\
\hline Demon & .60 & .65 & 4.70 & 2.56 & 5.04 & 8 & .30 & Grace & 4.67 & 1.78 & & & & A & .28 \\
\hline Devil & .93 & .87 & 5.63 & 2.13 & 6.94 & A & .31 & Grass & 3.25 & 1.79 & 6.63 & 6.96 & 7.54 & AA & .25 \\
\hline Dirt & 2.50 & 1.27 & 6.10 & 6.66 & 6.44 & 21 & .21 & Greed & 6.04 & 1.79 & 3.53 & 1.73 & 5.52 & 3 & .23 \\
\hline Doll & .00 & .00 & 6.17 & 6.94 & 6.12 & 46 & & Green & 4.20 & 1.58 & 6.60 & 5.46 & 1.99 & $\mathrm{AA}$ & .25 \\
\hline Dollar & 1.42 & .90 & 6.50 & 6.62 & 6.48 & $\mathrm{AA}$ & .36 & Grief & 2.65 & 1.17 & 4.70 & 1.86 & 6.32 & 45 & .26 \\
\hline Door & 7.10 & 1.81 & 6.60 & 7.00 & 7.96 & $\mathrm{AA}$ & .18 & Grove & 2.14 & 1.03 & & & & 31 & .24 \\
\hline Dove & 2.00 & 1.01 & 6.53 & 6.90 & 6.36 & 19 & .40 & Gun & 6.04 & 1.46 & & & & A & .40 \\
\hline Drama & 1.85 & 1.29 & 4.90 & 3.66 & 7.00 & 23 & .26 & Hall & 5.91 & 1.46 & 6.37 & 6.72 & 6.16 & $\mathrm{AA}$ & .33 \\
\hline Dream & 3.38 & 1.60 & 4.60 & 3.03 & 5.44 & $\mathrm{AA}$ & .22 & Hamlet & .00 & .00 & 5.87 & 6.19 & 6.56 & 15 & \\
\hline Dress & 2.95 & 1.20 & 6.53 & 6.93 & 5.68 & $\mathrm{AA}$ & .14 & Hammer & .70 & .98 & 6.73 & 6.96 & 6.92 & 34 & .12 \\
\hline Dumb & 4.13 & 1.79 & & & & 34 & .24 & Hard & 2.73 & 1.14 & & & & $\mathrm{AA}$ & .20 \\
\hline Dummy & 2.34 & 1.27 & 5.83 & 6.34 & 4.88 & 2 & .39 & Harp & 1.68 & .94 & 6.60 & 6.94 & 6.00 & 20 & .56 \\
\hline Dust & 4.77 & 1.51 & 6.03 & 6.67 & 6.84 & A & .40 & Hat & 6.51 & 1.73 & & & & $\mathrm{AA}$ & .33 \\
\hline Duty & .98 & .97 & 3.17 & 2.32 & 5.60 & $\mathrm{AA}$ & .20 & Haven & .70 & .46 & & & & 3 & .70 \\
\hline Earth & 2.49 & 1.26 & 6.27 & 6.58 & 7.56 & $\mathrm{AA}$ & .42 & Health & 1.00 & .82 & 4.10 & 3.54 & 6.39 & $\mathrm{AA}$ & .33 \\
\hline Edition & .00 & .00 & 3.40 & 4.64 & 5.88 & 19 & & Heap & 4.97 & 1.73 & & & & 46 & .33 \\
\hline Engine & .00 & .00 & 6.33 & 6.76 & 6.08 & A & & Hearing & 3.14 & 1.54 & 3.77 & 3.57 & 6.64 & 13 & .16 \\
\hline Errand & .00 & .00 & 4.27 & 4.21 & 6.48 & 20 & & Hermit & .43 & .50 & & & & 16 & .43 \\
\hline Error & 2.28 & 1.48 & & & & 36 & .09 & Hide & 4.49 & 1.70 & 3.80 & 5.45 & 5.63 & A & .13 \\
\hline Essence & .12 & .39 & 2.33 & 1.66 & 3.28 & 8 & .06 & Hint & 2.79 & 1.29 & 2.57 & 3.35 & 3.72 & 29 & .23 \\
\hline Event & 2.44 & 2.64 & 2.90 & 3.72 & 5.04 & A & .11 & History & .96 & .61 & 3.47 & 3.03 & 6.91 & $\mathrm{AA}$ & .48 \\
\hline Fable & 4.11 & .90 & & & & 13 & .46 & Hoax & 2.17 & 1.52 & & & & & .14 \\
\hline Fact & 2.84 & 1.77 & 2.20 & 3.31 & 4.29 & AA & .12 & Home & 2.56 & 1.36 & 6.50 & 6.25 & 6.88 & $\mathrm{AA}$ & .21 \\
\hline Fantasy & .00 & .00 & 3.70 & 2.03 & 5.06 & 4 & & Honor & .00 & .00 & 3.50 & 1.75 & 5.08 & $\mathrm{AA}$ & \\
\hline Farm & 1.87 & 1.19 & & & & $\mathrm{AA}$ & .47 & Hoof & 1.82 & 1.03 & 6.37 & 6.90 & 6.32 & 26 & .30 \\
\hline Fast & 3.31 & 1.24 & & & & AA & .25 & Hope & 5.00 & 1.43 & 3.83 & 1.18 & 5.52 & $\mathrm{AA}$ & .42 \\
\hline Fate & 5.78 & 1.58 & 2.37 & 1.46 & 4.00 & A & .23 & Horse & 2.33 & 1.29 & 6.80 & 6.94 & 8.67 & $\mathrm{AA}$ & .23 \\
\hline Fault & 2.27 & 1.24 & 2.83 & 2.87 & 4.80 & A & .32 & Hostage & .00 & .00 & 5.57 & 6.05 & 6.40 & 3 & \\
\hline Feast & 2.50 & 1.09 & & & & 45 & .23 & Hotel & .96 & .20 & 6.40 & 6.80 & 5.96 & A & .96 \\
\hline Feline & .60 & .50 & 4.67 & 5.31 & 5.42 & 2 & .60 & Hound & 5.00 & 1.53 & 6.27 & 6.83 & 6.00 & 23 & .28 \\
\hline
\end{tabular}

APPENDIX A (Continued) 
APPENDIX A (Continued)

\begin{tabular}{|c|c|c|c|c|c|c|c|}
\hline \multirow[b]{2}{*}{ Target Words } & \multicolumn{2}{|c|}{$R$} & \multirow[b]{2}{*}{$I$} & \multirow[b]{2}{*}{$C$} & \multirow[b]{2}{*}{$m$} & \multirow[b]{2}{*}{$F^{\mathrm{a}}$} & \multirow[b]{2}{*}{$O$} \\
\hline & $M$ & $S D$ & & & & & \\
\hline Hour & 3.04 & 1.52 & 3.60 & 2.93 & 5.56 & AA & .34 \\
\hline House & 2.74 & .98 & 6.67 & 6.93 & 6.83 & $\mathrm{AA}$ & .39 \\
\hline Humor & 1.13 & 1.27 & 4.57 & 2.31 & 5.72 & 41 & .23 \\
\hline Hurdle & 2.48 & 1.07 & 6.33 & 6.65 & 5.92 & 2 & .50 \\
\hline Idea & .10 & .31 & 2.20 & 1.42 & 4.88 & AA & .03 \\
\hline Injury & .00 & .00 & 6.37 & 6.87 & 6.96 & 20 & \\
\hline Ink & 5.56 & 1.70 & 6.37 & 6.87 & 6.96 & 20 & .33 \\
\hline $\operatorname{Inn}$ & 5.16 & 1.60 & 6.07 & 6.80 & 6.61 & 27 & .29 \\
\hline Insect & .00 & .00 & 6.10 & 6.80 & 6.32 & 40 & \\
\hline Interest & .00 & .00 & 3.13 & 2.20 & 5.52 & $\mathrm{AA}$ & \\
\hline Iron & .23 & .48 & 6.07 & 6.87 & 6.12 & AA & .11 \\
\hline Jail & 6.78 & 2.22 & 6.43 & 6.69 & 7.38 & 22 & .19 \\
\hline Jam & 4.29 & 1.49 & & & & 16 & .23 \\
\hline Jelly & 2.15 & .89 & 6.40 & 6.73 & 6.00 & 19 & .43 \\
\hline Joke & 3.31 & 1.45 & 4.27 & 3.80 & 5.92 & 32 & .21 \\
\hline Journal & .98 & .94 & 5.60 & 6.69 & 5.88 & AA & .14 \\
\hline Joy & 3.52 & 1.16 & 5.43 & 1.66 & 6.52 & AA & .24 \\
\hline Judge & 2.56 & 1.20 & 6.27 & 6.25 & 7.04 & $\mathrm{AA}$ & .32 \\
\hline Jump & 5.91 & 1.27 & & & & A & .33 \\
\hline Jury & 3.00 & 1.33 & 6.07 & 6.17 & 6.88 & 26 & .27 \\
\hline Keg & 3.05 & 1.22 & 6.40 & 6.87 & 4.84 & 3 & .38 \\
\hline Kettle & 1.93 & 1.18 & 6.23 & 7.00 & 7.44 & 27 & .39 \\
\hline Kine & 5.05 & 1.68 & 2.00 & 4.53 & 2.88 & 1 & .23 \\
\hline King & 4.82 & 1.30 & 6.27 & 6.34 & 7.50 & AA & .28 \\
\hline Kiss & 2.40 & 1.24 & 6.80 & 6.13 & 6.68 & AA & .27 \\
\hline Labor & 1.08 & .94 & & & & AA & .36 \\
\hline Lad & 6.05 & 1.36 & 6.33 & 6.58 & 5.76 & A & .40 \\
\hline Lake & 5.77 & 1.53 & 6.67 & 6.90 & 9.22 & AA & .26 \\
\hline Lark & 4.20 & 1.18 & 6.20 & 6.83 & 5.84 & 22 & .32 \\
\hline Lather & 1.16 & .89 & & & & 2 & .58 \\
\hline Law & 3.70 & 1.69 & 3.73 & 3.23 & 6.32 & $\mathrm{AA}$ & .21 \\
\hline Lawn & 2.50 & 1.18 & 6.57 & 6.96 & 7.28 & 37 & .28 \\
\hline Leader & 2.80 & 1.52 & 5.13 & 5.83 & 6.16 & $\mathrm{AA}$ & .12 \\
\hline Length & .51 & .50 & 3.73 & 3.75 & 5.84 & $\mathrm{AA}$ & .51 \\
\hline Leopard & .57 & .72 & 6.77 & 7.00 & 6.83 & 6 & .29 \\
\hline Letter & 2.67 & 1.26 & 6.37 & 6.94 & 5.96 & $\mathrm{AA}$ & .21 \\
\hline Lice & 3.95 & 1.15 & 5.57 & 6.36 & 6.56 & 1 & .25 \\
\hline Life & 2.10 & 1.13 & 4.07 & 2.96 & 6.78 & AA & .42 \\
\hline Limb & 2.49 & 1.66 & 6.17 & 6.93 & 6.80 & 38 & .17 \\
\hline Lime & 3.39 & 1.37 & 5.67 & 6.69 & 7.12 & 27 & .34 \\
\hline Link & 5.98 & 1.65 & 4.80 & 5.38 & 5.20 & 24 & .31 \\
\hline Lip & 5.94 & 2.02 & 6.57 & 6.93 & 5.32 & $\mathrm{AA}$ & .24 \\
\hline Lobster & .73 & .45 & 6.57 & 6.96 & 6.84 & 7 & .73 \\
\hline Lord & 3.48 & .99 & 4.63 & 4.18 & 6.68 & $\mathrm{AA}$ & .14 \\
\hline Lore & 6.22 & 2.03 & & & & 4 & .16 \\
\hline Love & 1.96 & .91 & 5.60 & 1.80 & 6.44 & AA & .39 \\
\hline Lump & 4.76 & 1.32 & 5.63 & 6.20 & 5.44 & 20 & .30 \\
\hline Madness & 2.14 & .89 & 4.03 & 2.35 & 5.16 & 12 & .71 \\
\hline Maiden & .58 & .55 & 6.10 & 6.52 & 5.04 & 45 & .58 \\
\hline Majority & 1.21 & .87 & 3.63 & 3.48 & 5.48 & A & .20 \\
\hline Maker & 2.50 & .83 & 3.57 & 4.46 & 5.00 & 27 & .36 \\
\hline Malice & 1.28 & 1.04 & 3.30 & 1.73 & 4.56 & 10 & .21 \\
\hline Mammal & .82 & .77 & 5.57 & 6.31 & 5.80 & 6 & .41 \\
\hline Mantle & .10 & .30 & 6.27 & 6.76 & 5.92 & 19 & .10 \\
\hline Market & .00 & .00 & 6.13 & 6.08 & 7.04 & AA & \\
\hline Marriage & .94 & .23 & 5.80 & 3.94 & 6.29 & A & .94 \\
\hline Mast & 3.41 & 1.50 & 6.43 & 6.96 & 7.12 & 26 & .28 \\
\hline Master & 2.14 & 1.06 & 4.97 & 5.53 & 5.28 & AA & .27 \\
\hline Meat & 5.24 & 1.20 & 6.63 & 6.93 & 8.32 & AA & .20 \\
\hline Meeting & 2.60 & 1.03 & 5.20 & 4.80 & 5.20 & $\mathrm{AA}$ & .15 \\
\hline Memory & .16 & .53 & 3.10 & 1.78 & 5.00 & A & .16 \\
\hline Menace & .87 & .84 & 3.73 & 3.70 & 5.04 & 21 & .29 \\
\hline Metal & 2.16 & 1.00 & 5.87 & 6.76 & 6.96 & A & .54 \\
\hline Middle & 2.00 & 1.10 & & & & AA & .33 \\
\hline Miracle & .76 & .80 & 3.33 & 2.25 & 5.60 & 24 & .15 \\
\hline Mirage & 1.35 & .65 & 4.97 & 3.50 & 5.63 & 1 & .27 \\
\hline Mirth & 2.12 & .99 & & & & 16 & .30 \\
\hline Missile & 1.33 & 1.08 & 6.33 & 6.80 & 6.48 & 1 & .15 \\
\hline
\end{tabular}

APPENDIX A (Continued)

\begin{tabular}{|c|c|c|c|c|c|c|c|}
\hline \multirow[b]{2}{*}{ Target Words } & \multicolumn{2}{|c|}{$R$} & \multirow[b]{2}{*}{$I$} & \multirow[b]{2}{*}{$C$} & \multirow[b]{2}{*}{$m$} & \multirow[b]{2}{*}{$F^{\mathrm{a}}$} & \multirow[b]{2}{*}{$O$} \\
\hline & $M$ & $S D$ & & & & & \\
\hline Mist & 2.74 & 1.51 & & & & 29 & .18 \\
\hline Moment & .00 & .00 & 2.50 & 2.52 & 4.38 & $\mathrm{AA}$ & \\
\hline Monarch & .00 & .00 & 6.20 & 6.40 & 6.92 & 20 & \\
\hline Money & 3.26 & 1.08 & 6.43 & 6.63 & 6.68 & $\mathrm{AA}$ & .47 \\
\hline Monk & 3.87 & 1.61 & 6.40 & 6.63 & 5.92 & 20 & .23 \\
\hline Mood & 2.78 & 1.81 & 3.67 & 1.52 & 5.36 & 27 & .13 \\
\hline Moral & 1.70 & 1.04 & 3.17 & 1.39 & 6.44 & A & .28 \\
\hline Moss & 3.45 & 1.40 & 6.33 & 6.87 & 7.04 & 22 & .38 \\
\hline Mother & 2.26 & 1.33 & 6.67 & 6.52 & 5.83 & A & .38 \\
\hline Mountain & 1.00 & .41 & 6.77 & 7.00 & 7.58 & $\mathbf{A A}$ & 1.00 \\
\hline Mucus & .43 & .68 & 5.53 & 6.16 & 6.38 & 1 & .43 \\
\hline Mule & .60 & .93 & 6.60 & 6.96 & 6.12 & 29 & .20 \\
\hline Nail & 5.84 & 2.18 & 6.50 & 6.96 & 6.08 & A & .19 \\
\hline Nation & 1.84 & 1.37 & & & & AA & .08 \\
\hline Nectar & 1.52 & 1.07 & 5.33 & 6.41 & 6.92 & 2 & .13 \\
\hline Noose & 2.92 & 1.24 & 6.23 & 6.20 & 6.20 & 2 & .29 \\
\hline Number & 1.34 & 1.03 & & & & $\mathrm{AA}$ & .27 \\
\hline Nun & 6.40 & 1.83 & 6.67 & 6.76 & 6.60 & 9 & .38 \\
\hline Nymph & .39 & .49 & 5.63 & 4.40 & 5.36 & 13 & .39 \\
\hline Ocean & 2.54 & 1.15 & 6.77 & 6.90 & 8.76 & $\mathrm{AA}$ & .42 \\
\hline Odor & 1.15 & 1.22 & 5.13 & 5.83 & 5.76 & 27 & .16 \\
\hline Opinion & .20 & .40 & 3.23 & 2.29 & 4. & AA & .10 \\
\hline Origin & .00 & .00 & 2.30 & 3.25 & 5. & 48 & \\
\hline Oven & .00 & .00 & 6.40 & 6. & 8 & 29 & \\
\hline Owner & 1.58 & 1.06 & 4.23 & 5.90 & 5. & A & .26 \\
\hline Pact & 2.59 & 1.81 & 3.50 & 3.77 & 5.12 & 4 & .14 \\
\hline Pail & 6.98 & 1.90 & & & & 16 & .19 \\
\hline Painter & .00 & .00 & 5.87 & 6.59 & 6.40 & 25 & \\
\hline Palace & 1.80 & 1.21 & 6.50 & 6.73 & 7.08 & A & .26 \\
\hline Panic & .87 & .97 & 5.33 & 2,18 & 6.72 & 19 & .08 \\
\hline Pants & 1.90 & 1.30 & & & & 6 & .13 \\
\hline Paper & 1.54 & .98 & 6.30 & 6.89 & 7.68 & $\mathrm{AA}$ & .19 \\
\hline Party & .70 & .66 & 6.27 & 5.50 & 7.08 & AA & .35 \\
\hline Passio & 1.23 & .72 & 5.30 & 1.66 & 5.68 & A & .41 \\
\hline Paste & 2.74 & .98 & & & & 17 & .27 \\
\hline Peach & 3.97 & 1.42 & 6.60 & 6.80 & 6.84 & 29 & .28 \\
\hline Pelt & 3.96 & 1.23 & 5.40 & 6.20 & 6.76 & 6 & .40 \\
\hline Pep & .88 & 1.01 & 4.60 & 2.73 & 6.04 & 3 & .22 \\
\hline Pepper & .60 & .73 & 6.27 & 6.96 & 6.52 & 27 & .30 \\
\hline Person & .04 & .20 & 5.83 & 6.51 & 5.68 & AA & .04 \\
\hline Pig & 4.60 & 1.48 & & & & 44 & .39 \\
\hline Pipe & 1.84 & 1.33 & 6.43 & 6.90 & 6.20 & A & .20 \\
\hline Plain & 5.37 & 2.08 & 5.37 & 5.28 & 5.20 & $\mathrm{AA}$ & .14 \\
\hline Plank & 4.95 & 1.45 & 6.30 & 6.96 & 6.20 & 19 & .25 \\
\hline Plant & 1.60 & 1.05 & 6.50 & 6.87 & 8.32 & $\mathrm{AA}$ & .20 \\
\hline Plea & 5.33 & 2.50 & & & & 10 & .16 \\
\hline Pledge & 2.12 & 1.08 & 3.63 & 2.93 & 5.92 & 27 & .30 \\
\hline Pole & 5.06 & 1.72 & 0 & 6.93 & 6.20 & A & .27 \\
\hline Portal & .86 & .51 & 10 & 6.25 & 5. & 7 & .29 \\
\hline Poster & 2.82 & 1.17 & 3 & 6.96 & 0 & 2 & .47 \\
\hline Potato & .77 & .43 & 0 & 7.00 & 7.13 & A & .77 \\
\hline Powe & 2.90 & 1.48 & 4.47 & 2.73 & 5.88 & AA & .32 \\
\hline Prairie & 4.46 & 2.00 & 6.47 & 6.80 & 8.16 & 25 & .15 \\
\hline Present & 1.13 & .88 & 4.80 & 3.88 & 6.13 & $\mathrm{AA}$ & .38 \\
\hline Press & .06 & .30 & 4.10 & 3.63 & 5.80 & 48 & .03 \\
\hline Preview & .96 & .62 & 4.03 & 4.14 & 4.12 & 1 & .48 \\
\hline Pride & 5.24 & 1.73 & 4.23 & 1.49 & 5.24 & A & .18 \\
\hline Priest & 2.78 & 1.48 & 6.53 & 6.59 & 6.88 & 42 & .21 \\
\hline Prison & .33 & .61 & 6.23 & 6.62 & 7.21 & A & .16 \\
\hline Proxy & 1.26 & .76 & 2.70 & 3.72 & 4.84 & 1 & .21 \\
\hline Python & .06 & .23 & 5.47 & 6.42 & 5.88 & 1 & .06 \\
\hline Quest & 5.28 & 2.14 & 4.53 & 2.76 & 5.20 & 20 & .20 \\
\hline Rabbit & .76 & .59 & & & & 43 & .38 \\
\hline Rage & 3.94 & 1.15 & & & & 49 & .44 \\
\hline Rattle & 3.26 & 1.02 & 6.30 & 6.60 & 6.80 & 28 & .36 \\
\hline Rear & 5.89 & 2.20 & & & & A & .21 \\
\hline Reflex & .48 & .71 & 4.73 & 4.08 & 5.88 & 2 & .03 \\
\hline Research & .00 & .00 & 4.13 & 3.22 & 6.92 & 22 & \\
\hline
\end{tabular}


APPENDIX A (Continued)

\begin{tabular}{|c|c|c|c|c|c|c|c|}
\hline \multirow[b]{2}{*}{ Target Words } & \multicolumn{2}{|c|}{$R$} & \multirow[b]{2}{*}{$I$} & \multirow[b]{2}{*}{$C$} & \multirow[b]{2}{*}{$m$} & \multirow[b]{2}{*}{$F^{\mathrm{a}}$} & \multirow[b]{2}{*}{$O$} \\
\hline & $M$ & $S D$ & & & & & \\
\hline Resident & 1.32 & 1.18 & & & & 14 & .12 \\
\hline Riddle & 2.91 & 1.07 & & & & 12 & .32 \\
\hline $\operatorname{Rim}$ & 4.21 & 1.47 & & & & 12 & .23 \\
\hline Ring & 4.88 & 1.64 & & & & $\mathrm{AA}$ & .27 \\
\hline River & 2.03 & 1.17 & 6.63 & 6.83 & 7.52 & $\mathrm{AA}$ & .34 \\
\hline Robber & 1.92 & 1.00 & 5.67 & 6.25 & 6.88 & 27 & .02 \\
\hline Robbery & .28 & .50 & 5.00 & 4.75 & 6.16 & 9 & .14 \\
\hline Rock & 5.16 & 1.98 & 6.37 & 6.96 & 5.96 & $\mathrm{AA}$ & .29 \\
\hline Rod & 4.00 & 1.58 & 5.97 & 6.62 & 6.04 & 44 & .33 \\
\hline Roof & 1.62 & 1.26 & & & & AA & .32 \\
\hline Rosin & .00 & .00 & 4.20 & 5.88 & 4.56 & 1 & \\
\hline Rot & 5.72 & 2.29 & & & & 8 & .27 \\
\hline Rubble & 2.63 & 1.22 & 5.23 & 6.18 & 5.40 & 1 & .53 \\
\hline Rug & 5.80 & 1.65 & & & & 40 & .36 \\
\hline Sadness & 2.08 & .85 & 4.63 & 2.47 & 5.56 & 11 & .69 \\
\hline Salad & 1.00 & .76 & 6.53 & 6.83 & 7.20 & 28 & .25 \\
\hline Salary & .40 & .50 & 4.70 & 5.23 & 5.08 & A & .40 \\
\hline Saloon & 2.56 & 1.55 & 6.43 & 6.70 & 7.12 & 12 & .12 \\
\hline Salve & .40 & .50 & & & & 3 & .20 \\
\hline Sauce & 3.26 & 1.67 & 5.80 & 6.76 & 5.88 & 27 & .41 \\
\hline Science & 1.08 & .98 & 4.07 & 3.05 & 6.56 & A & .15 \\
\hline Sea & 5.41 & 2.04 & 6.73 & 6.79 & 7.16 & $\mathrm{AA}$ & .22 \\
\hline Search & 1.95 & 1.00 & & & & A & .49 \\
\hline Season & 1.45 & .74 & 4.87 & 4.58 & 7.88 & AA & .72 \\
\hline Seat & 5.60 & 1.26 & 6.40 & 6.79 & 6.08 & AA & .20 \\
\hline Sentry & .78 & .72 & & & & 5 & .26 \\
\hline Serf & .80 & .70 & 5.50 & 6.03 & 6.75 & 4 & .27 \\
\hline Series & .17 & .43 & 4.47 & 3.88 & 5.36 & A & .08 \\
\hline Session & .53 & .80 & 3.67 & 3.62 & 5.40 & 19 & .05 \\
\hline Shame & 5.21 & 1.58 & 4.10 & 1.70 & 5.16 & A & .33 \\
\hline Shift & 2.40 & 1.47 & & & & 35 & .24 \\
\hline Ship & 5.32 & 2.08 & 6.67 & 6.93 & 7.87 & AA & .22 \\
\hline Shock & 5.29 & 1.82 & 4.67 & 3.97 & 6.20 & A & .33 \\
\hline Shoes & 3.19 & 1.53 & 6.63 & 7.00 & 7.52 & $\mathrm{AA}$ & .11 \\
\hline Shore & 6.54 & 2.26 & 6.63 & 6.69 & 8.13 & $\mathrm{AA}$ & .18 \\
\hline Shove & 2.13 & .94 & & & & 15 & .43 \\
\hline Shriek & 3.24 & 2.12 & 5.73 & 5.70 & 6.92 & 26 & .15 \\
\hline Shrub & 4.10 & 1.57 & & & & 17 & .24 \\
\hline Shy & 7.36 & 2.28 & & & & 21 & .24 \\
\hline Sight & 6.16 & 1.65 & & & & $\mathrm{AA}$ & .25 \\
\hline Silence & .20 & .41 & 4.10 & 3.09 & 5.82 & AA & .20 \\
\hline Singer & 2.67 & 1.27 & 6.17 & 6.35 & 6.64 & 20 & .24 \\
\hline Skin & 4.63 & 1.95 & 6.43 & 6.96 & 5.67 & AA & .27 \\
\hline Skull & 2.18 & 1.77 & 6.47 & 6.96 & 6.64 & 12 & .31 \\
\hline Sky & 6.54 & 2.60 & 6.73 & 6.18 & 7.84 & $\mathrm{AA}$ & .13 \\
\hline Slain & 4.80 & 1.89 & & & & 27 & .13 \\
\hline Slave & 4.16 & 1.68 & 6.23 & 6.38 & 6.17 & A & .28 \\
\hline Slush & 3.79 & 1.51 & 6.27 & 6.65 & 6.80 & 1 & .38 \\
\hline Smell & 5.43 & 2.23 & & & & A & .29 \\
\hline Snake & 6.40 & 1.79 & 6.90 & 7.00 & 6.88 & 28 & .29 \\
\hline Soil & 4.43 & 1.13 & 6.00 & 6.87 & 8.00 & AA & .40 \\
\hline Song & 2.12 & 1.12 & & & & $\mathrm{AA}$ & .27 \\
\hline Soul & 3.77 & 1.91 & 2.13 & 1.87 & 6.40 & $\mathrm{AA}$ & .20 \\
\hline Sour & 1.28 & 1.04 & & & & 15 & .18 \\
\hline Speaker & 2.92 & 1.55 & 5.67 & 6.13 & 5.54 & 25 & .20 \\
\hline Speech & 4.14 & 1.42 & 4.33 & 4.87 & 5.36 & $\mathrm{AA}$ & .30 \\
\hline Spice & 5.08 & 1.21 & & & & 17 & .30 \\
\hline Spire & 4.80 & 2.10 & 5.57 & 6.72 & 4.72 & 11 & .16 \\
\hline Spray & 6.00 & 2.60 & 6.17 & 6.21 & 6.48 & 22 & .16 \\
\hline Spree & 6.47 & 2.58 & 4.27 & 2.81 & 5.79 & 2 & .21 \\
\hline Square & 6.18 & 2.46 & 6.37 & 5.70 & 5.44 & $\mathrm{AA}$ & .17 \\
\hline Star & 5.07 & 1.26 & 6.70 & 6.73 & 7.04 & AA & .42 \\
\hline Steam & 3.80 & 1.44 & 6.37 & 6.41 & 7.46 & A & .21 \\
\hline Steamer & 2.21 & 1.42 & 6.53 & 6.94 & 6.32 & 28 & .25 \\
\hline Stone & 4.34 & 1.46 & 6.23 & 6.96 & 7.24 & $\mathrm{AA}$ & .15 \\
\hline Store & 5.11 & 2.58 & & & & AA & .14 \\
\hline Storm & 2.60 & 1.35 & 6.43 & 6.45 & 7.21 & $\mathrm{AA}$ & .52 \\
\hline Stream & 4.09 & 1.56 & & & & AA & .26 \\
\hline
\end{tabular}

APPENDIX A (Continued)

\begin{tabular}{|c|c|c|c|c|c|c|c|}
\hline \multirow[b]{2}{*}{ Target Words } & \multicolumn{2}{|c|}{$R$} & \multirow[b]{2}{*}{$I$} & \multirow[b]{2}{*}{$C$} & \multirow[b]{2}{*}{$m$} & \multirow[b]{2}{*}{$F^{\mathrm{a}}$} & \multirow[b]{2}{*}{$O$} \\
\hline & $M$ & $S D$ & & & & & \\
\hline Street & 5.32 & 1.59 & 6.57 & 6.62 & 7.48 & $\mathrm{AA}$ & .22 \\
\hline Strength & .80 & .40 & 4.63 & 2.90 & 5.12 & AA & .80 \\
\hline Stub & 4.51 & 1.56 & 4.97 & 6.20 & 5.24 & 5 & .30 \\
\hline Student & .62 & .49 & 6.27 & 6.38 & 5.96 & A & .62 \\
\hline Style & 4.05 & 1.48 & 3.83 & 3.18 & 5.84 & A & .24 \\
\hline Suds & 1.08 & .63 & 6.40 & 6.75 & 7.29 & 3 & .18 \\
\hline Sugar & .00 & .00 & 6.57 & 6.96 & 7.00 & AA & \\
\hline Swamp & .70 & .76 & 6.33 & 6.62 & 6.67 & 29 & .14 \\
\hline Table & 3.85 & 1.39 & 6.50 & 7.00 & 7.60 & $\mathrm{AA}$ & .43 \\
\hline Tank & 5.00 & 1.59 & 6.23 & 6.87 & 6.48 & 19 & .25 \\
\hline Temple & .00 & .00 & 6.13 & 6.69 & 6.75 & A & \\
\hline Theory & .00 & .00 & 2.57 & 1.90 & 5.88 & A & \\
\hline Thicket & 1.61 & 1.00 & 5.60 & 6.49 & 6.04 & 19 & .32 \\
\hline Thief & 2.58 & 1.43 & 5.70 & 6.08 & 6.50 & 28 & .29 \\
\hline Thorn & 4.81 & 1.26 & 6.33 & 6.87 & 6.44 & 24 & .27 \\
\hline Thought & 3.95 & 1.40 & 2.77 & 1.28 & 5.32 & AA & .40 \\
\hline Ticket & 1.86 & 1.01 & 6.20 & 7.00 & 7.21 & A & .37 \\
\hline Time & 2.81 & .80 & 4.13 & 2.47 & 7.00 & AA & .26 \\
\hline Tire & 3.40 & 1.40 & & & & AA & .20 \\
\hline Toast & 4.18 & 1.38 & 6.57 & 6.93 & 7.84 & 20 & .60 \\
\hline Tomb & 3.14 & 1.46 & 6.37 & 6.73 & 6.04 & 22 & .26 \\
\hline Tool & 3.78 & 1.33 & 5.77 & 6.80 & 6.88 & 40 & .25 \\
\hline Tower & 2.46 & 1.16 & 6.53 & 6.96 & 6.42 & A & .20 \\
\hline Town & 1.98 & 1.51 & & & & AA & .25 \\
\hline Toy & 3.59 & 1.22 & 6.17 & 6.63 & 6.96 & 49 & .20 \\
\hline Tract & 1.52 & 1.05 & 4.77 & 4.14 & 6.40 & 1 & .30 \\
\hline Trash & 6.42 & 1.83 & & & & 3 & .34 \\
\hline Tree & 5.94 & 2.89 & 6.77 & 7.00 & 6.79 & AA & .20 \\
\hline Trellis & .14 & .35 & 5.60 & 6.69 & 5.00 & 2 & .07 \\
\hline Trick & 5.38 & 1.93 & & & & A & .24 \\
\hline Trip & 5.82 & 2.30 & & & & AA & .24 \\
\hline Troops & 3.02 & 1.44 & 6.13 & 6.69 & 7.54 & A & .28 \\
\hline Trouble & 2.24 & 1.13 & 3.53 & 2.25 & 5. & AA & .56 \\
\hline Truce & 3.57 & 1.53 & 4.80 & 3.05 & 6.17 & 3 & .19 \\
\hline Truck & 4.98 & 1.68 & 6.60 & 7.00 & 6.48 & 23 & .33 \\
\hline Trumpet & .58 & .50 & 6.60 & 7.00 & 6.80 & 17 & .58 \\
\hline Truth & 2.02 & 1.21 & 2.73 & 1.69 & 4.78 & $\mathrm{AA}$ & .22 \\
\hline Turn & 3.08 & 1.24 & & & & $\mathrm{AA}$ & .26 \\
\hline Twe & 1.55 & .89 & 6.57 & 6.93 & 5.80 & 1 & .22 \\
\hline Typhoon & 2.14 & 1.69 & 5.50 & 6.20 & 6.28 & & .07 \\
\hline Unit & .00 & .00 & 2.87 & 4.33 & 4.80 & 29 & \\
\hline Vacuum & .45 & .88 & 4.77 & 3.87 & 5.94 & 5 & .05 \\
\hline Valley & 2.29 & 1.16 & 6.57 & 6.66 & 6.56 & AA & .38 \\
\hline Vapor & 1.63 & 1.07 & 4.80 & 5.93 & 5.76 & 27 & .20 \\
\hline Venom & .53 & .65 & 4.23 & 5.62 & 6.40 & 5 & .53 \\
\hline Vessel & .80 & .88 & 5.90 & 6.99 & 7.82 & A & .26 \\
\hline Vest & 4.78 & 2.21 & 6.37 & 6.73 & 5.96 & 21 & .18 \\
\hline Vigor & 2.15 & 1.62 & 4.43 & 2.60 & 5.72 & 19 & .24 \\
\hline Village & 1.33 & 1.03 & 6.50 & 6.69 & 5.32 & AA & .33 \\
\hline Vision & .95 & .95 & 4.73 & 4.00 & 6.39 & 45 & .19 \\
\hline Water & .44 & .69 & 6.60 & 6.96 & 7.50 & $\mathrm{AA}$ & .06 \\
\hline Weak & 4.22 & 1.57 & & & & A & .18 \\
\hline Whale & 6.87 & 1.99 & 6.50 & 6.96 & 7.24 & 8 & .22 \\
\hline Wheat & 5.47 & 1.53 & 6.40 & 7.00 & 7.96 & A & .17 \\
\hline Wife & 2.30 & 1.07 & 6.53 & 6.52 & 6.48 & $\mathrm{AA}$ & .46 \\
\hline Window & .00 & .00 & 6.37 & 7.00 & 6.76 & AA & \\
\hline Wine & 4.75 & 1.72 & 6.60 & 6.96 & 7.54 & A & .19 \\
\hline Winter & .55 & .60 & 6.53 & 5.83 & 8.32 & AA & .09 \\
\hline Wish & 2.13 & .96 & & & & $\mathrm{AA}$ & .53 \\
\hline World & 1.16 & 1.15 & 5.97 & 5.90 & 6.88 & $\mathrm{AA}$ & .17 \\
\hline Wrench & 2.43 & 1.34 & & & & 11 & .30 \\
\hline Yacht & 3.18 & 2.10 & 6.77 & 6.96 & 7.20 & 2 & .17 \\
\hline
\end{tabular}

Note- $I, C$, and $m$ values were taken from Paivio, Yuille, and Madigan (1968). $F$ values were taken from Thorndike and Lorge (1944).

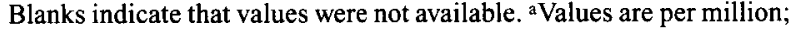
less than one per million are listed as $0 ; \mathrm{A} \geq 50<100 /$ million, $\mathrm{AA} \geq$ $100 /$ million. 


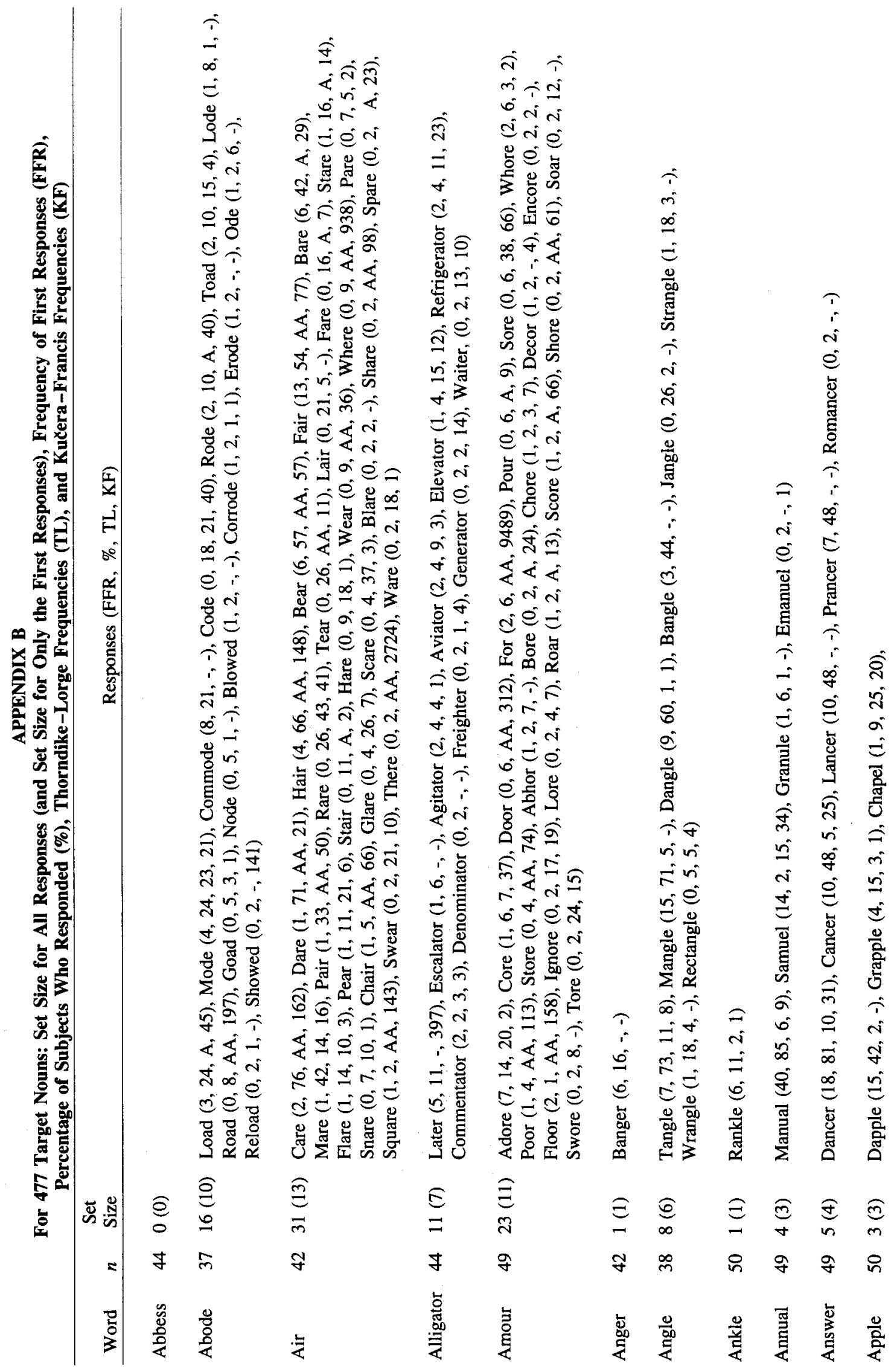




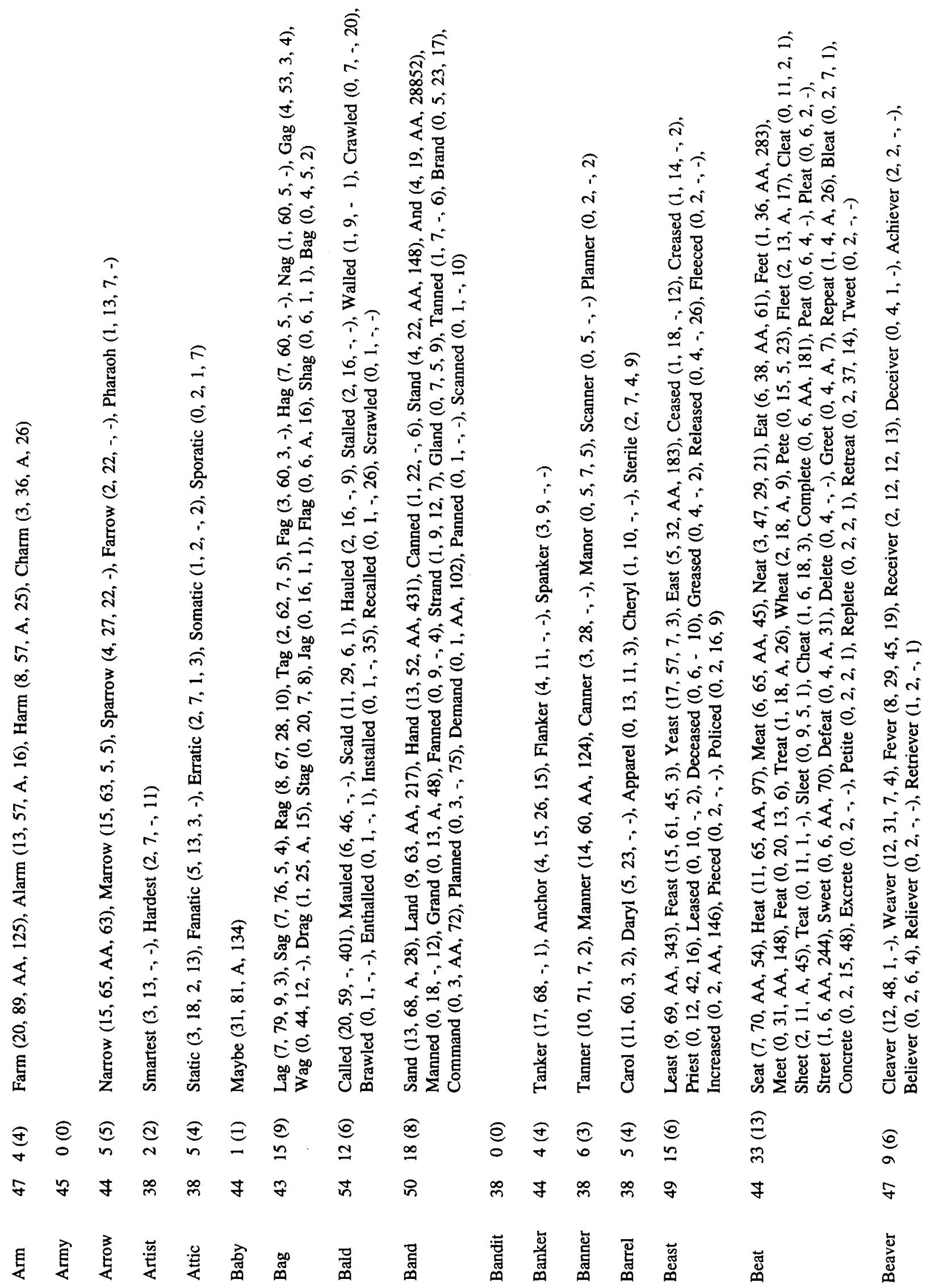




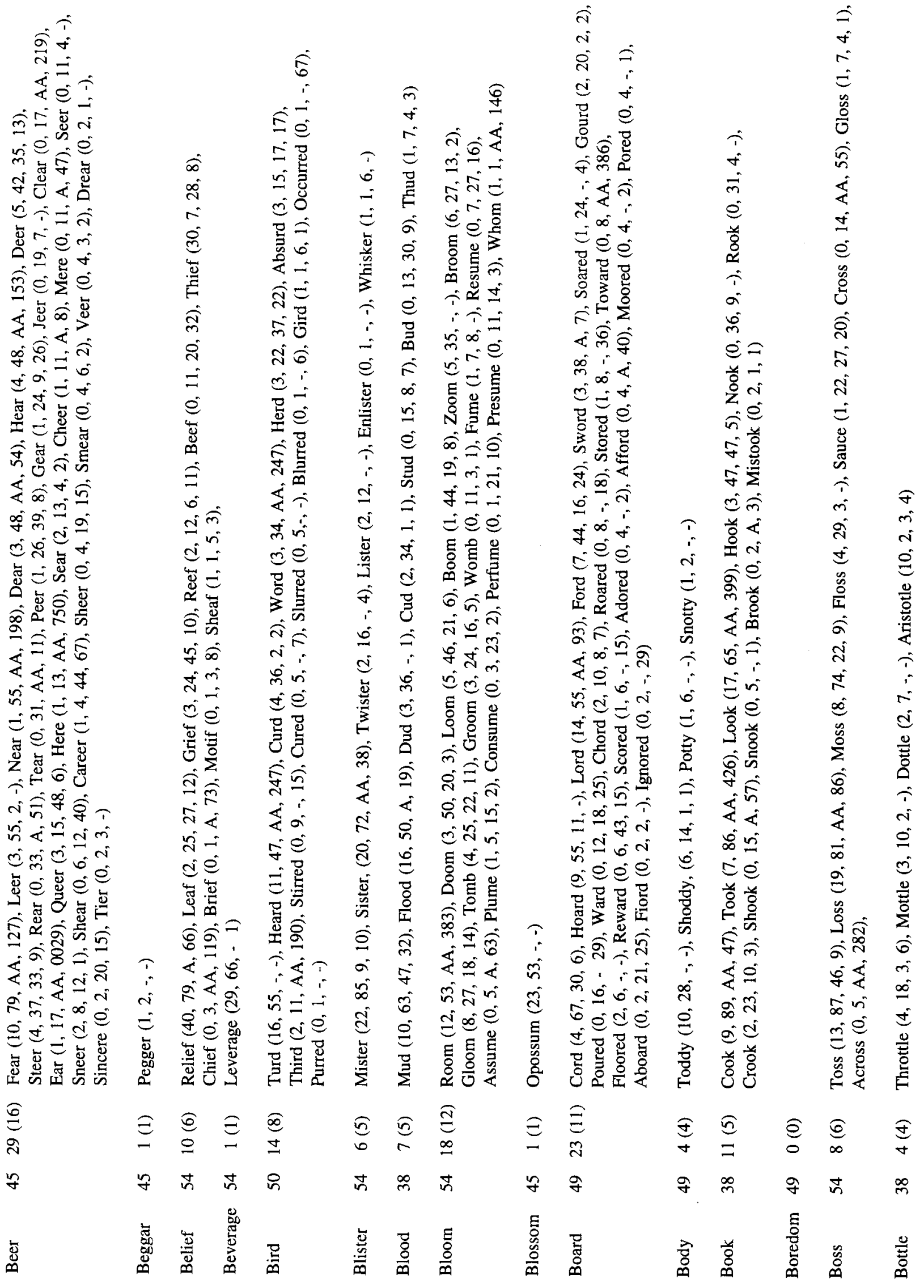




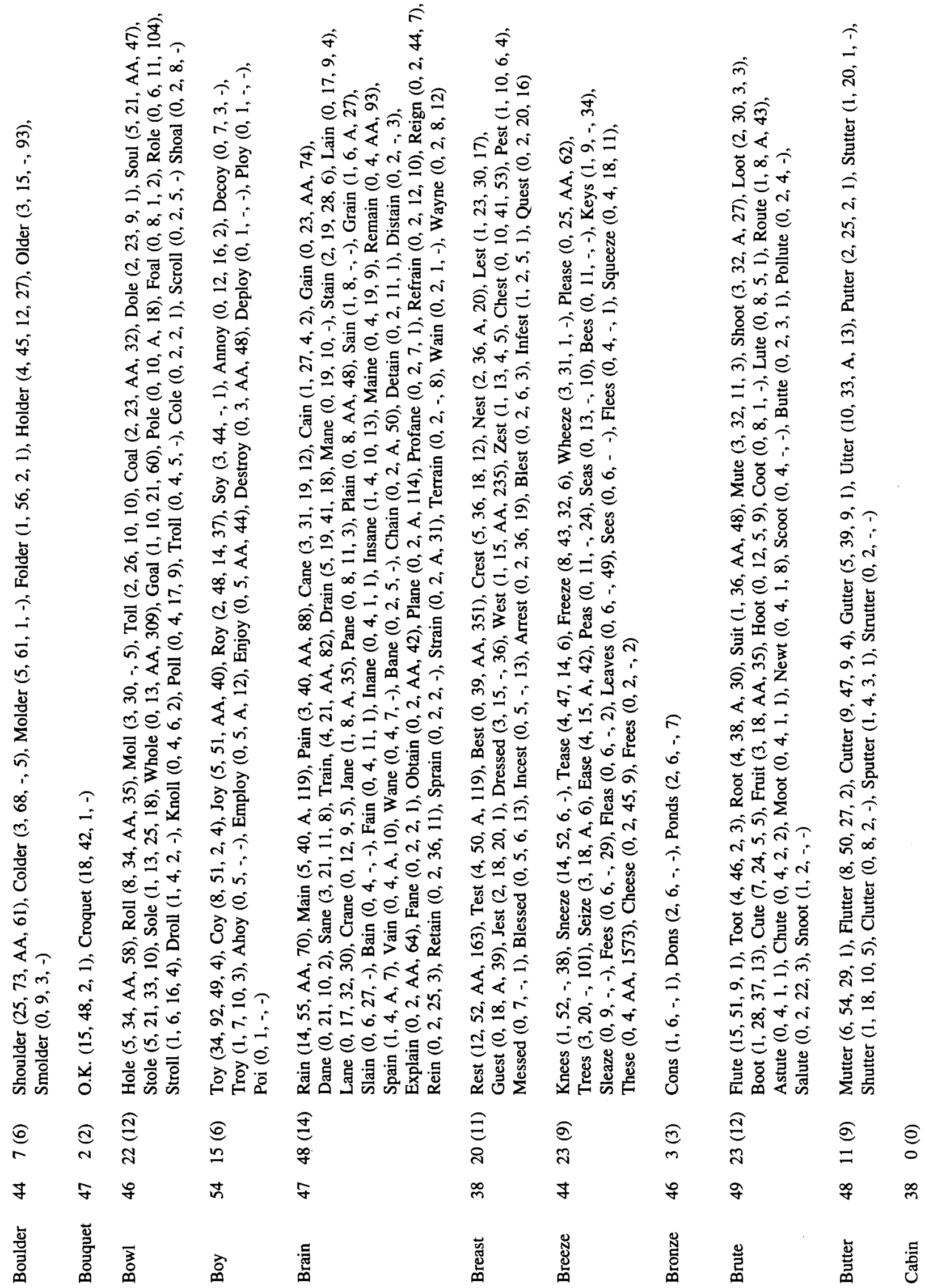


$\begin{array}{llll}\dot{i} & \ddot{r} & \dot{\sigma} & \dot{\sigma} \\ \dot{\sigma} & \dot{\sigma} & \dot{\sigma}\end{array}$

$\dot{0} \quad \dot{0} \quad \dot{0} \quad \dot{0}$

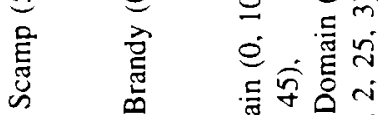

$\dot{\infty}$

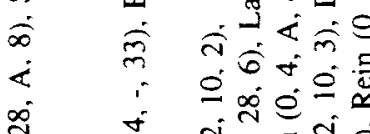

-

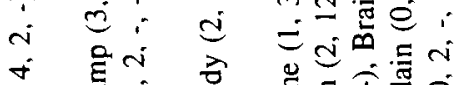

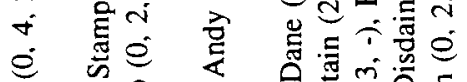

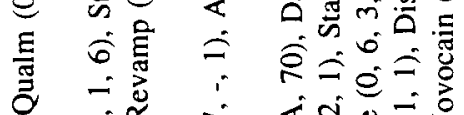

तi

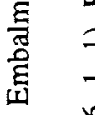

\%

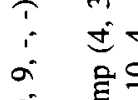

要$$
\text { (a) }
$$

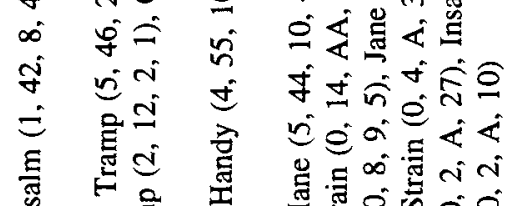

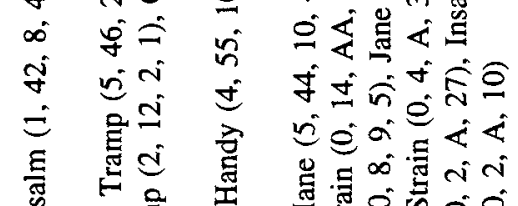

今.

व कि

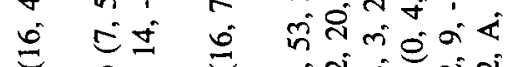

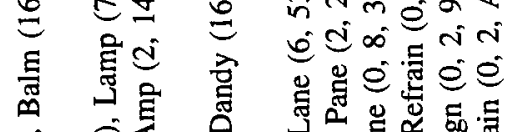

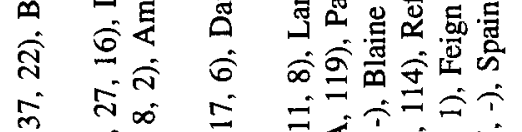

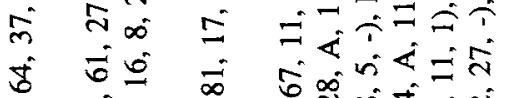

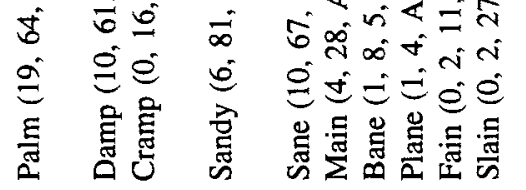

ล $\widehat{a} \bar{g}$

$0 \div$

\& $g \stackrel{\infty}{q}$ o

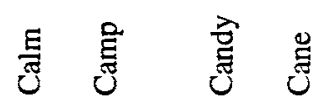

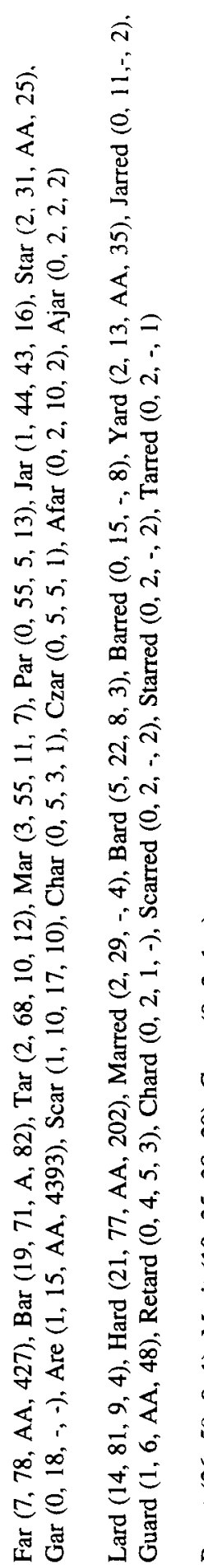

官

i $\dot{\vec{c}} \dot{m}$

i $\quad$ i

$=\quad \dot{e} \stackrel{=}{=}$

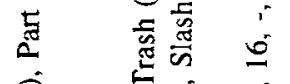

in $\quad \dot{\bar{N}}=$

$\sum$ करेष

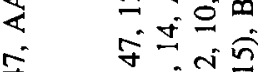

ষ $\quad \dot{e} \hat{e}=$

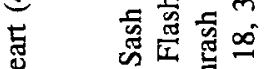

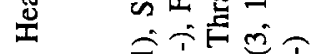

萑 तi

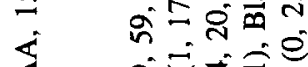

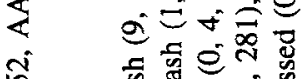

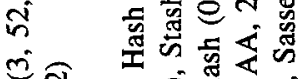

氙

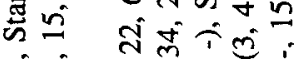

$\therefore a$ वृत्तें

$\therefore=$ बं

is

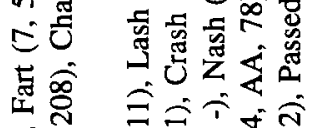

i

m

में

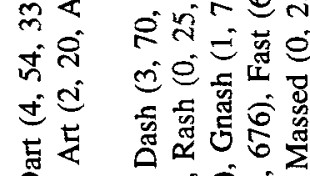

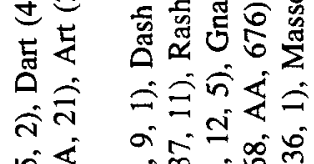

ติ

$\infty$

壱壱

$\hat{F} \div$

$\mathrm{Ni}$

요

êt

$$
\text { 西 }
$$

iñ cิง

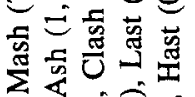

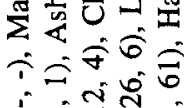
करों

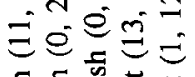

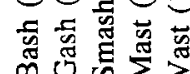

\section{$\dot{\imath}$}

i

$e_{\infty}^{i n} \quad \overline{0}$

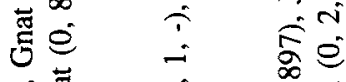

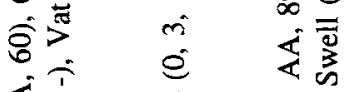

$\& !$

$-\infty$

过

ธิธี่

过

6อ

E

氠

6ำ

in

लi

¿̊ं

苟苛

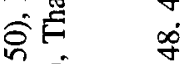

寸ำ

$\underbrace{\infty}<$

$\overline{\mathrm{o}} \mathrm{i}$

é

过 $\overline{\mathscr{Q}}$

这

8

¿̇

ప

ลे

ติ

$8 \dot{8}$

vi

昰

के

$=\dot{\theta}$

8

$\stackrel{\infty}{\infty}$

造

vi

踏 กิ

i $\infty$ क

我市

mi

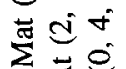

क

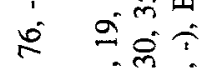

iี

को

三0

苟泀

灵

㐫 สू

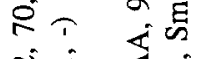

$\stackrel{2}{=}<$

焉

品 $\overline{0}$

$\infty \bar{\infty}$ 盛

यล

ㄴ.

¿ิ

包

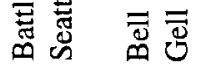

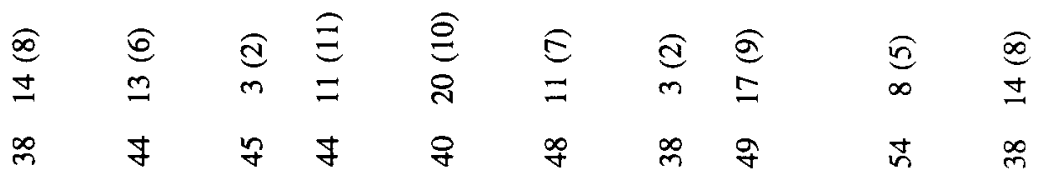

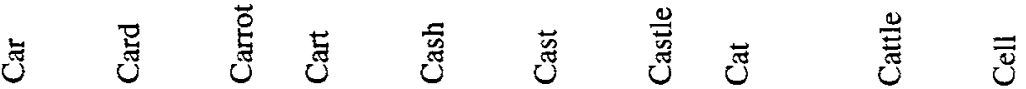




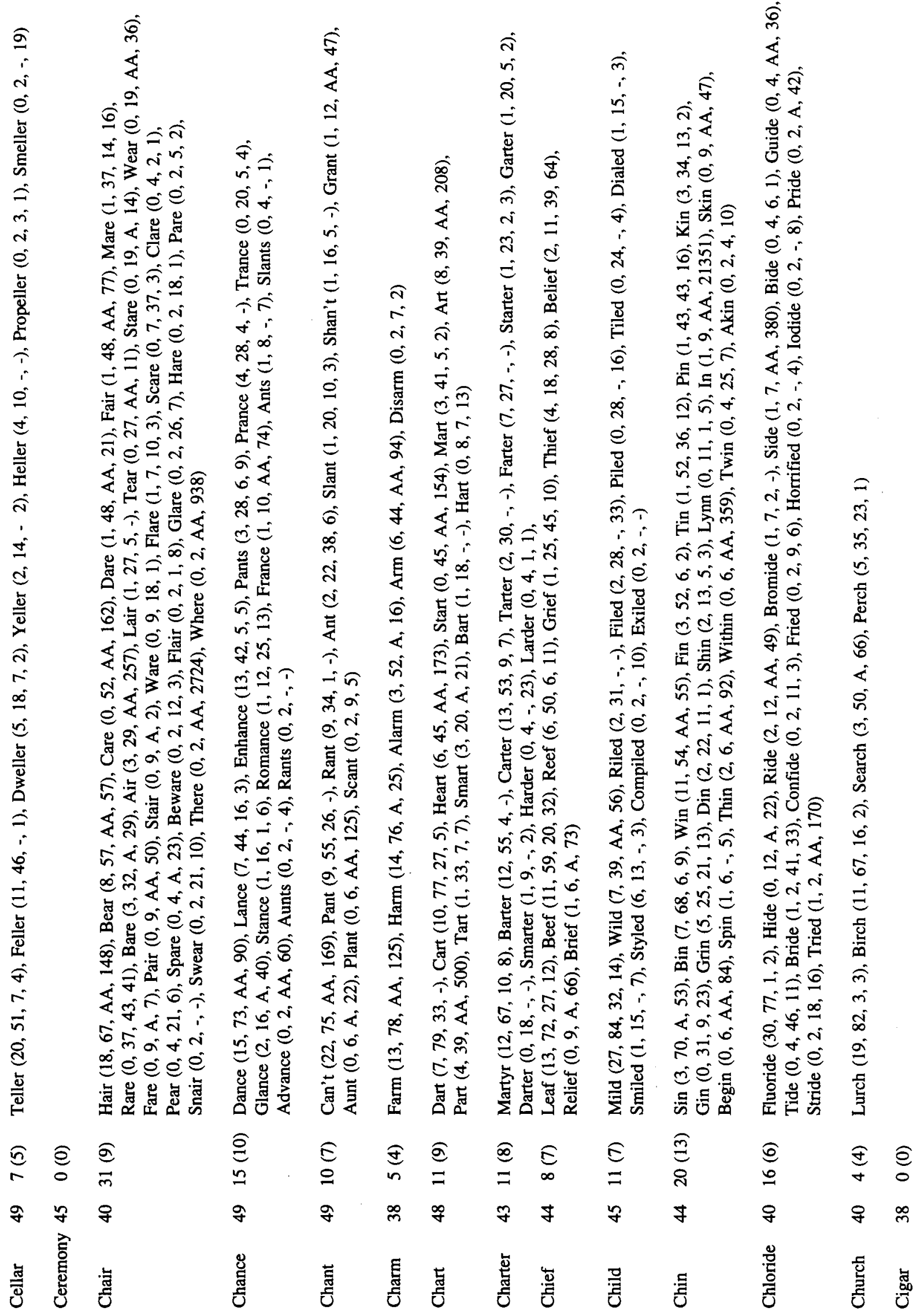




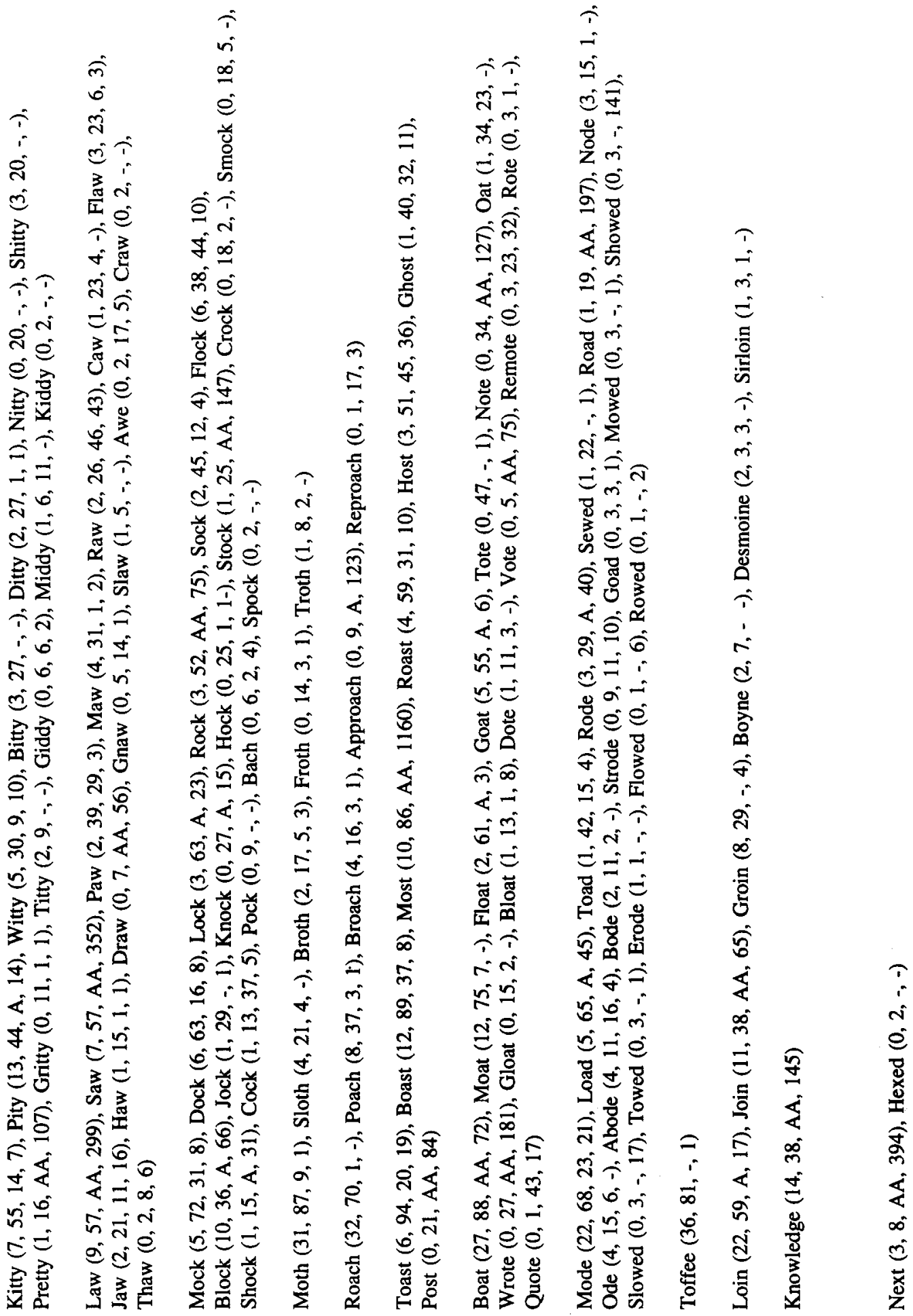
$\begin{array}{lllll}\varrho & \varrho & \varrho & \varrho & \Xi \\ 0 & 0 & \cong & \cong & \infty\end{array}$
过
$\Xi$

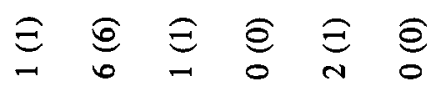
于 $\infty$
Ұ

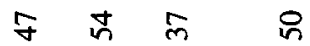
寸䓃子守
遦

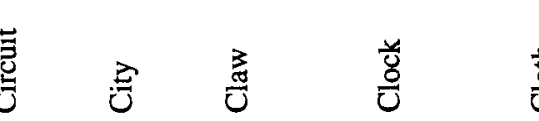

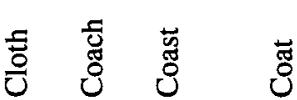
รัด
衰喜 


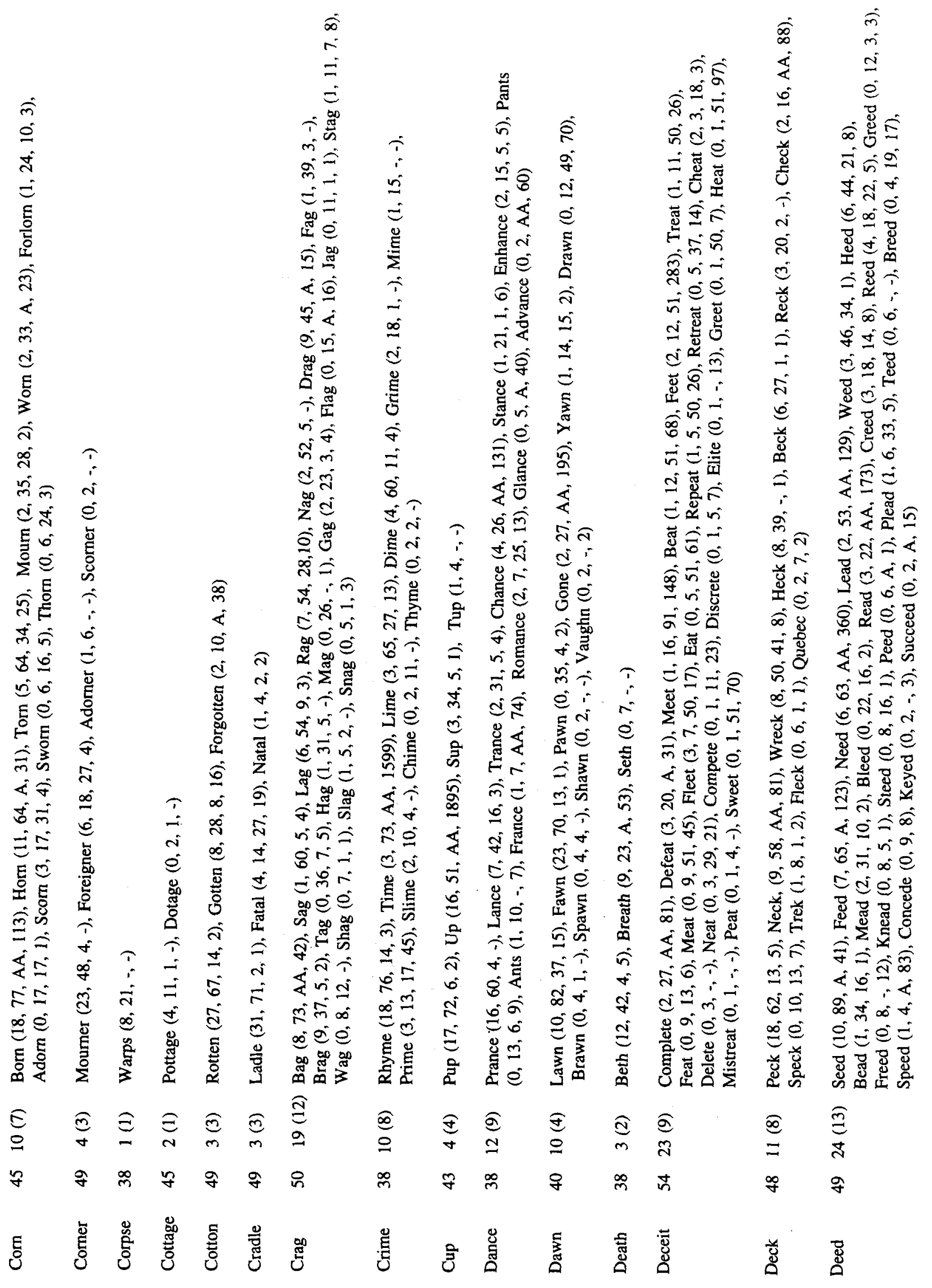




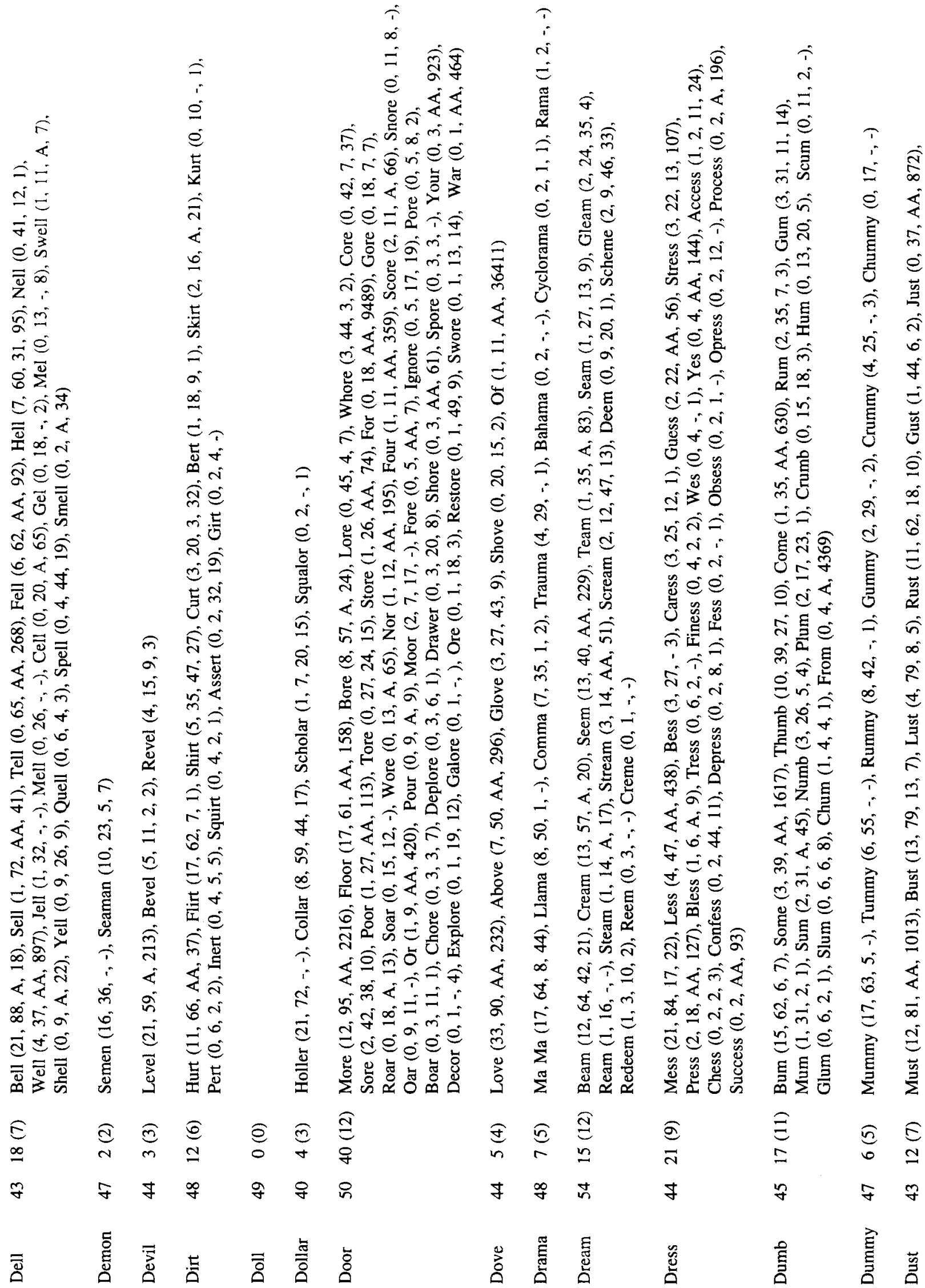




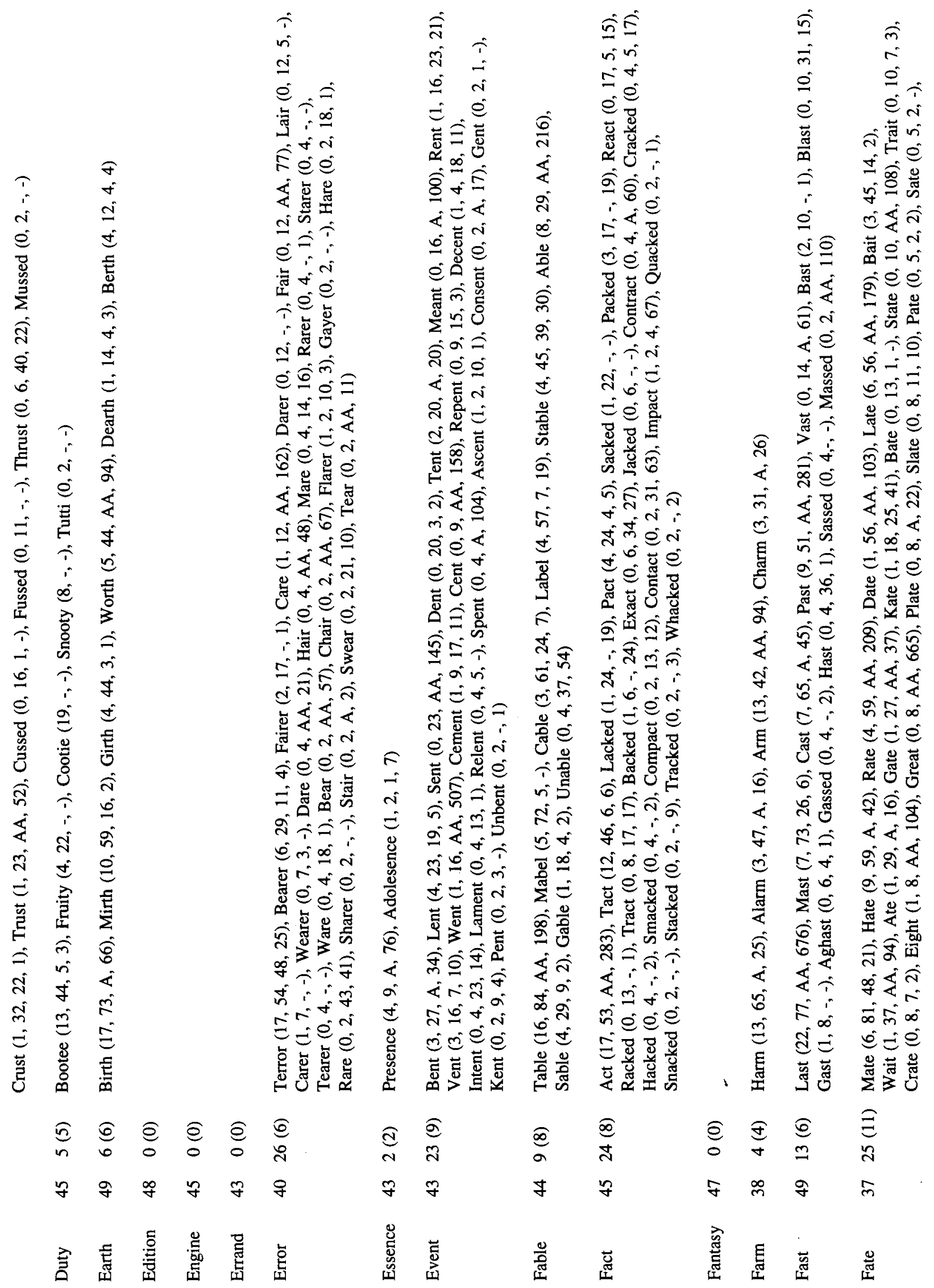




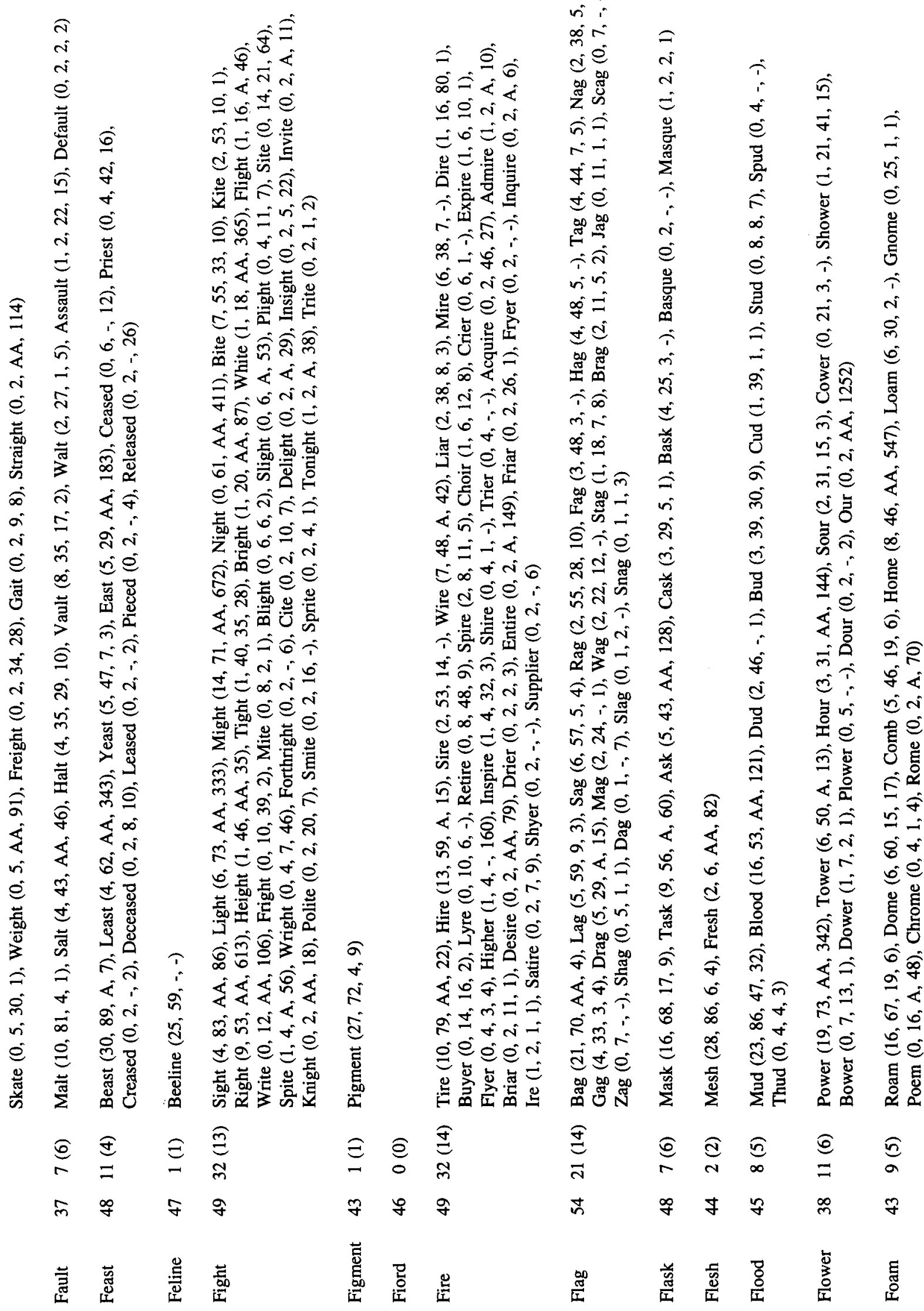




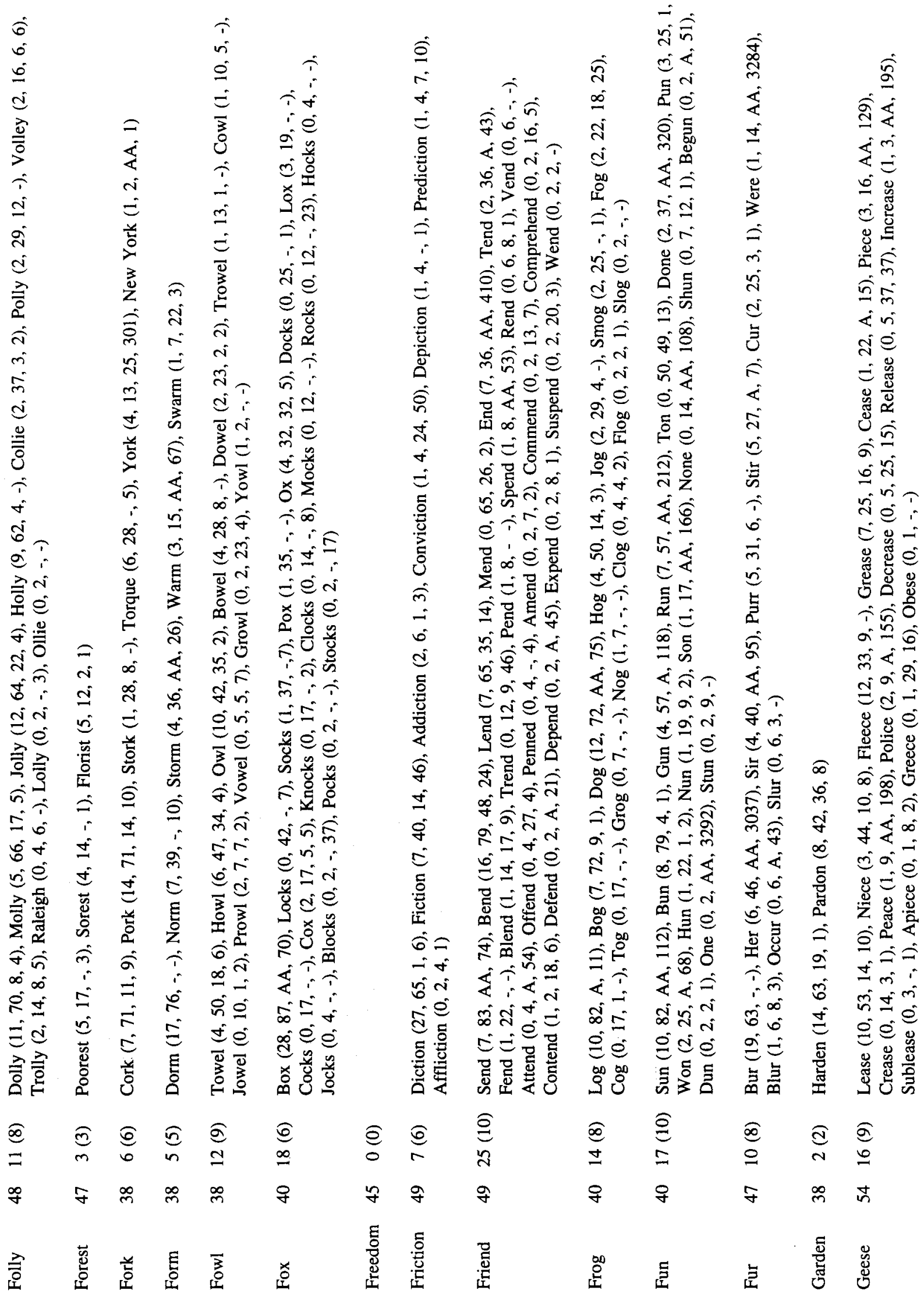




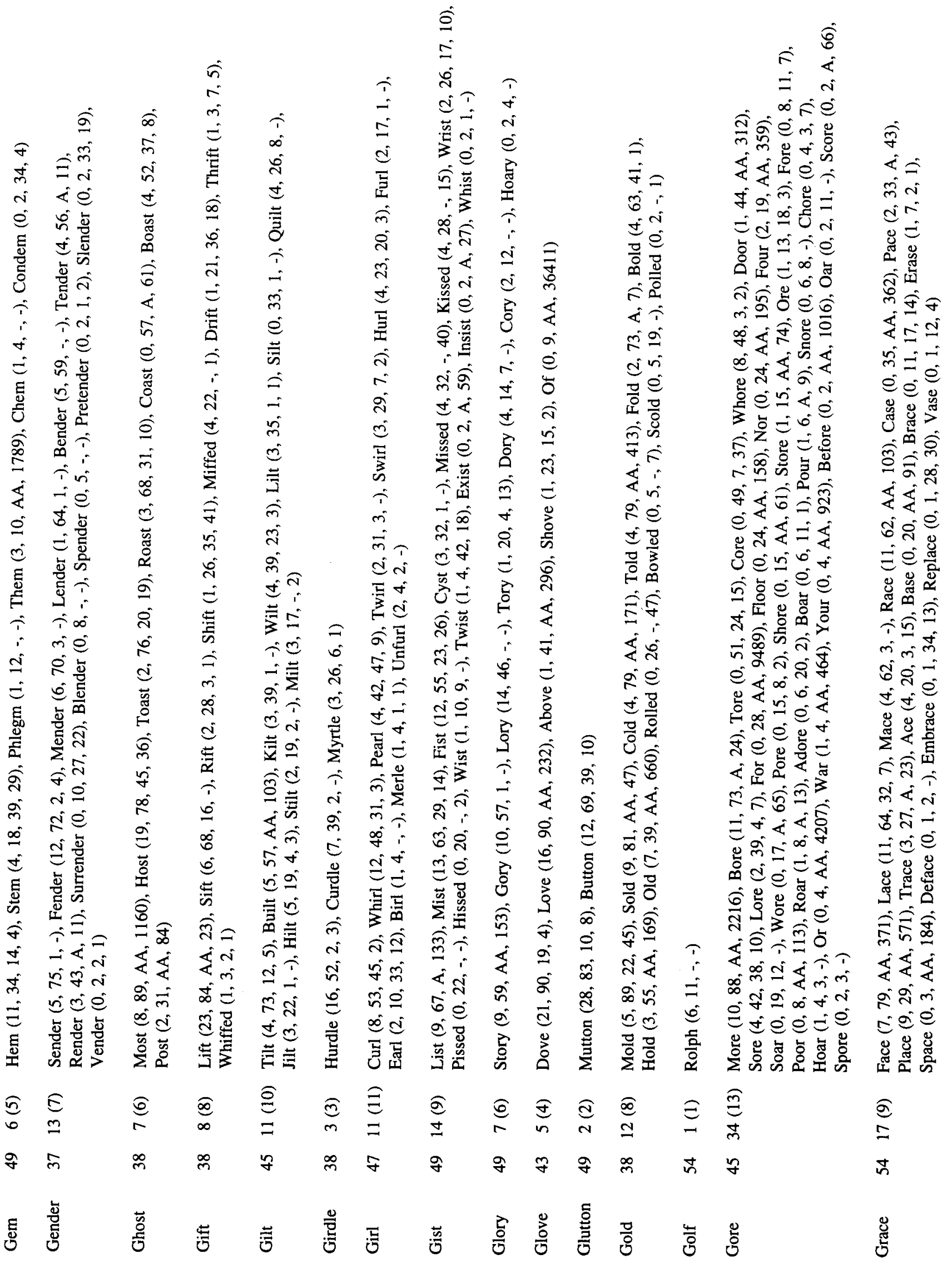




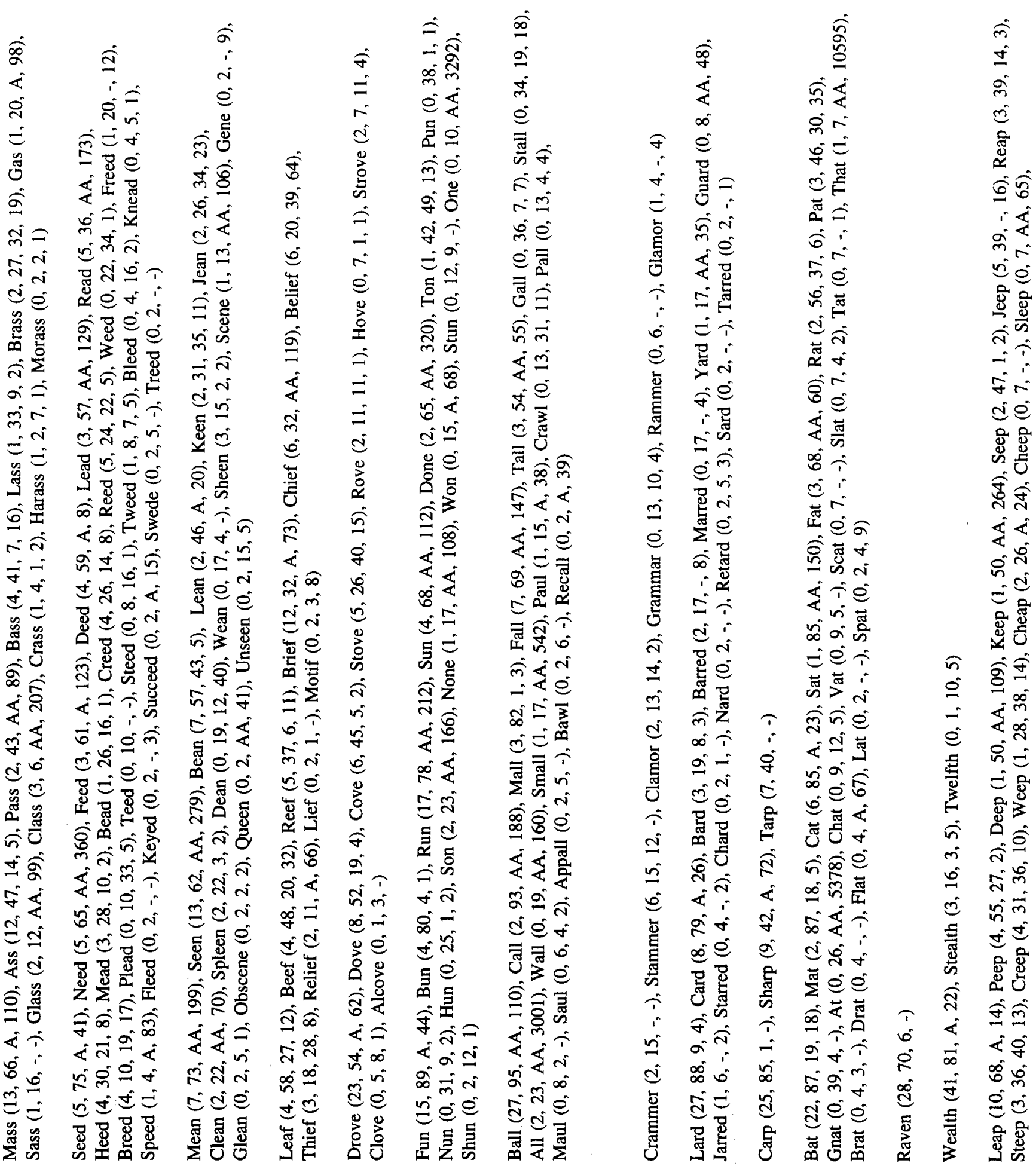

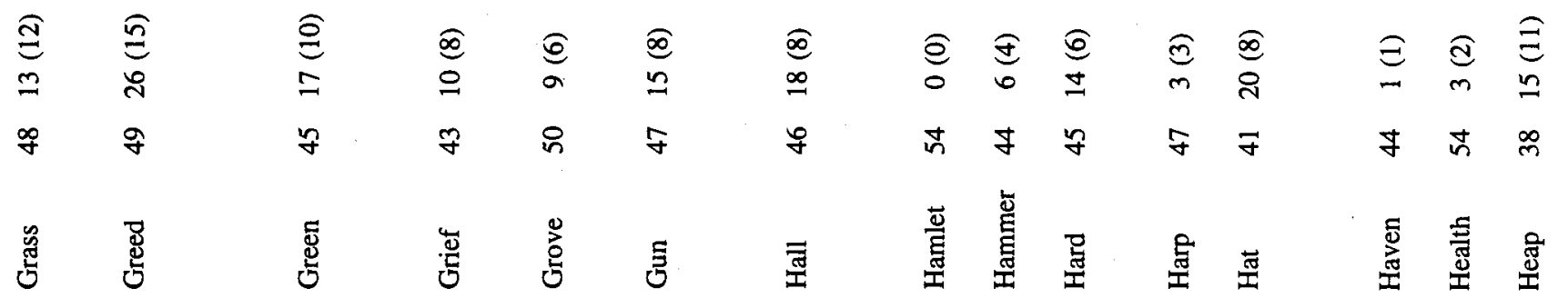




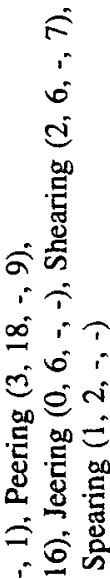

हों

é

on

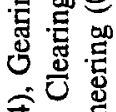

वें

के :

$\mathrm{d}=\mathrm{i}$

an

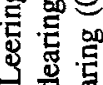

ลิ

$\therefore \div$

संत?

$\exists 0^{\circ}$

$=\sum_{0}^{\infty}$

量

蹗

ล ลิ่

$\therefore$ :

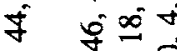

i

e $\quad \underbrace{\infty}_{00}$

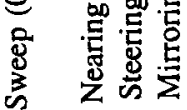

ลิ ลิก

$\approx$ ก $i$ ?

\& nंष

in ñé

é

क्षे

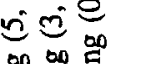

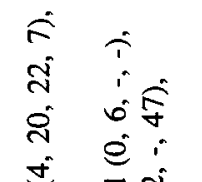

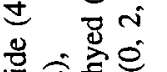

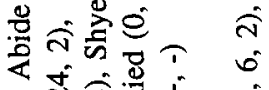

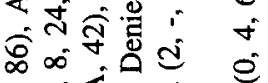

$\therefore e^{\dot{\alpha}} \hat{\mathrm{N}}$

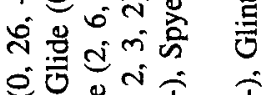

टण पूi i

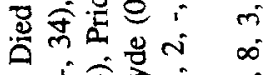

ㅇㅎㅇ

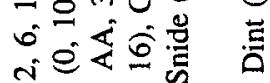

पु 000 क

हैं

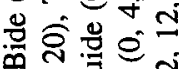

苛范

क्षे की

$0=0$

过它市

पि चुं

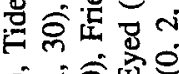

6ิ<安嘼

$\therefore \dot{\mathbb{S}}$

iखल

व菏

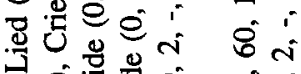

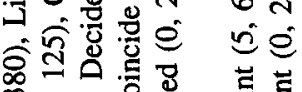

这它焉

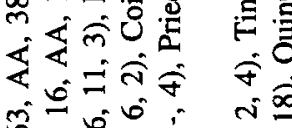

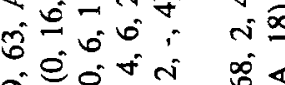

a $e$ :

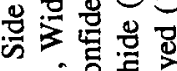

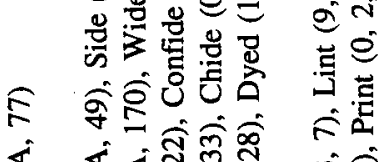

ป \&यत

जि $\infty+5$

nं

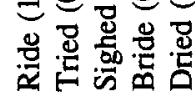

壱蓄

(

$\infty<$

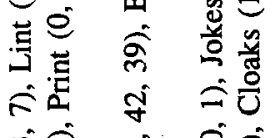

m?

$\infty+$

ते

章言

$i \div$

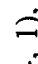

0

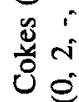

i

$\infty$

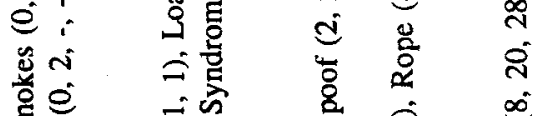

की ते ते की

if $\quad \overrightarrow{0} i$ i

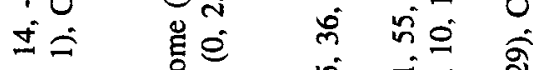

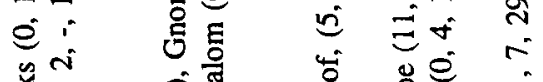

常 仓

कि

$\dot{ \pm} \widehat{\widehat{I}}$

?:

递

के

更

का

it

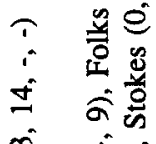

लि:

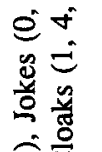

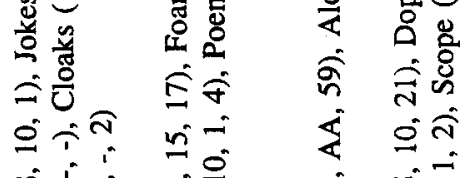

舟方

กी 0

స్ల
के

ㅇํㅇ

过乐

है ก

6

2

方

$\Xi \dot{0}$

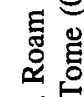

递

家察

$\overline{4}<$

求

ié

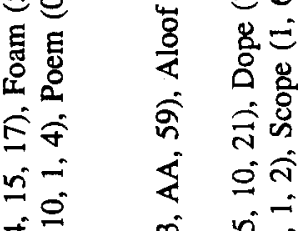

क

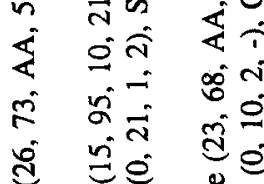

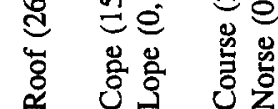

愘

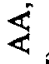

กี

요

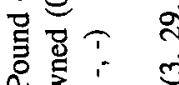

के

sin

\&े

चु

言登

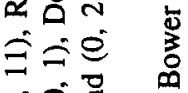

元要

e्य

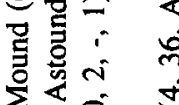

$\sum \dot{e} \pm$

金家卷

\&文号

त闭

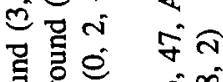

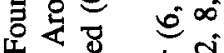

守完 है

<这政

$\therefore \infty \div$ किष्ठ

¿ह่ जิ

प⿺

范茟

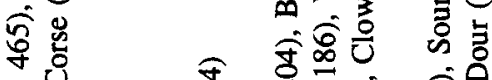

合总 की की

记

离

मे min

य

s $\propto$ U 0

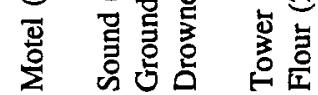

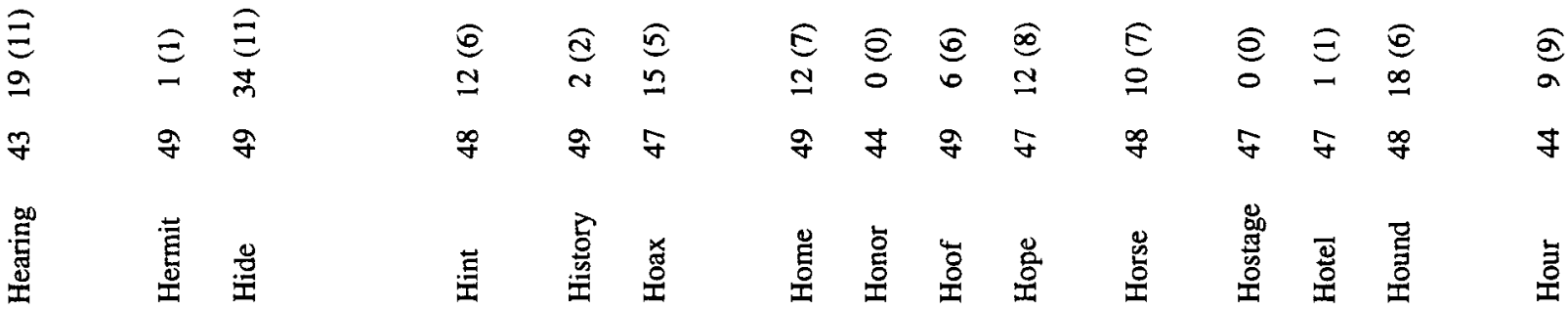




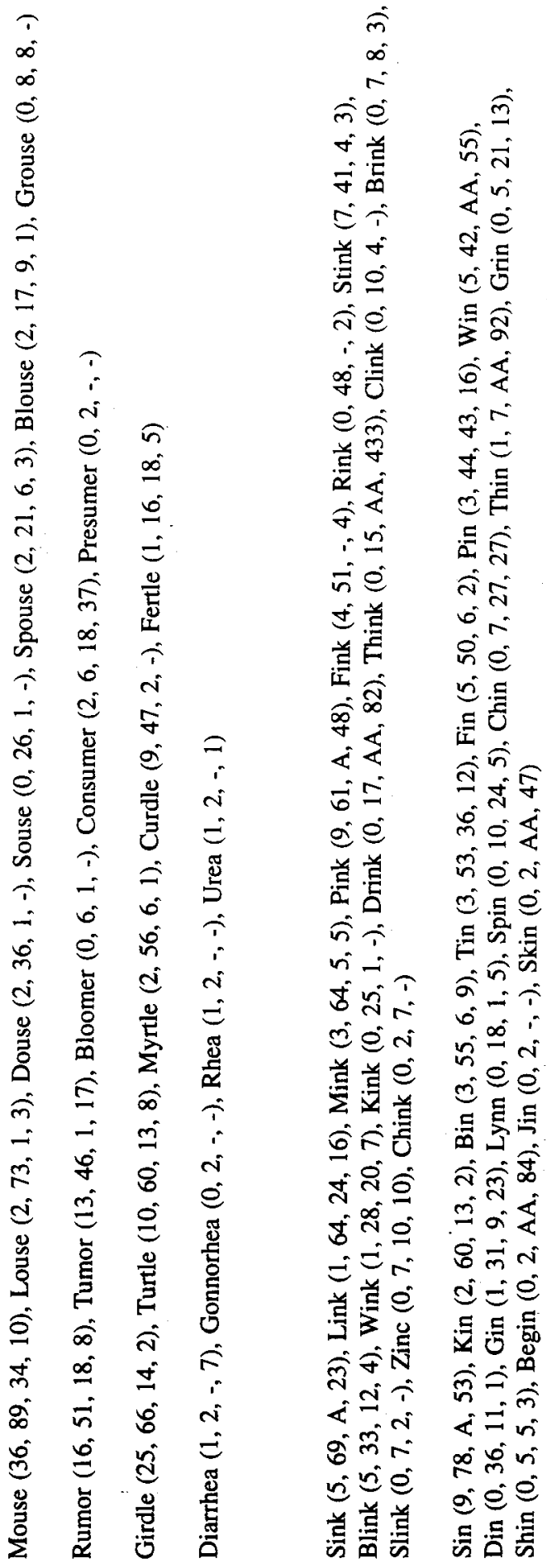

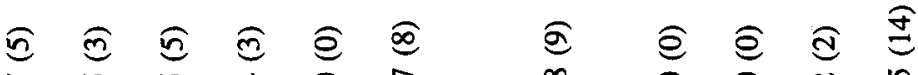

ๆ $\stackrel{\infty}{+}$ 吊

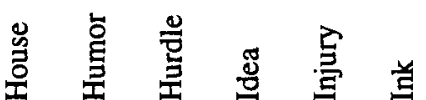

年 $\dot{i}$

$\therefore \quad \hat{e}$

क्ष

过

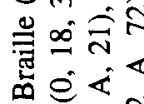

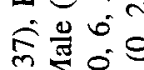

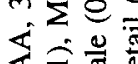

这苋

舟实安

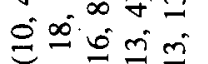

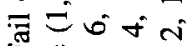

I.

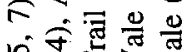

क⿺⿻一𠃋十

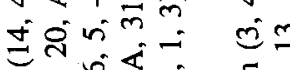

जि

mi

en

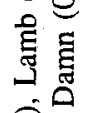

ลิ่

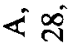

$\infty$

é

艾

$=6$

m

$\pm=$

纯

它

采

ลิ

$\infty$

守守

a $4 \pm$

ลี

¿อ

弟

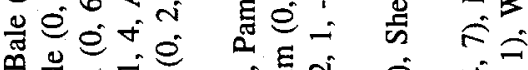

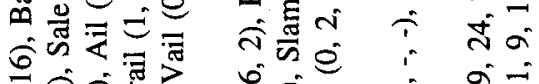

的霄方

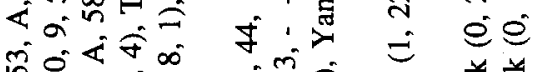

in $\infty 0^{\circ}$ i

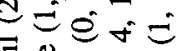

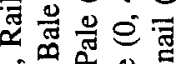

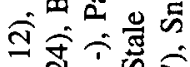

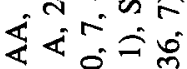

in

cंe

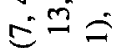

E्छे

约

คิ

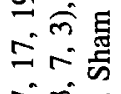

于ें

é :

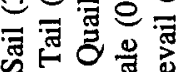

焉焉

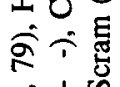

6)

तi

舟证=

तें

$\therefore \dot{0}=0$

E n

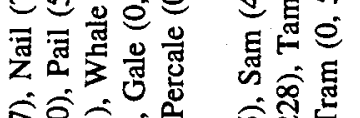

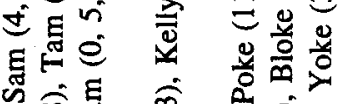

㐘

च

$\sum$

ลิ่

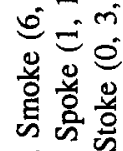

Iิ $\dot{-}$

$\therefore i m$

$+\frac{0}{\square}$

คิ่

ชิต

娄定

बंष्यें

$\infty \infty^{\infty}$

c ते

i $\quad \dot{E}=0$

苍咅咅

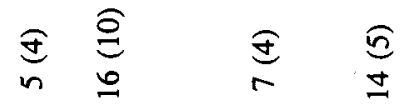

$\infty$

\&

ซ

in

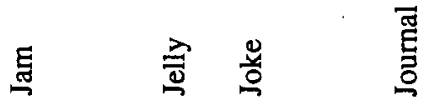




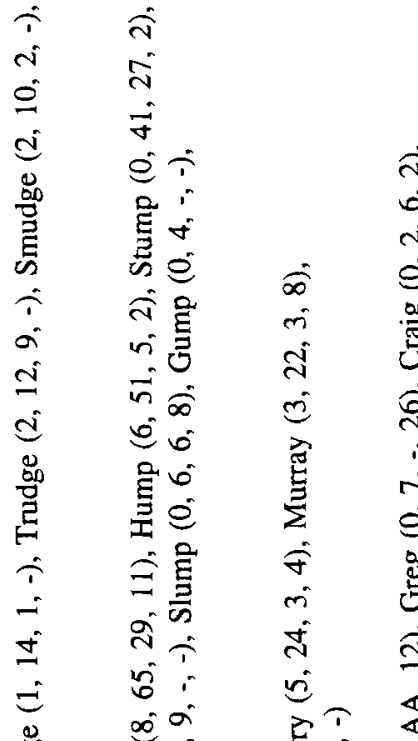

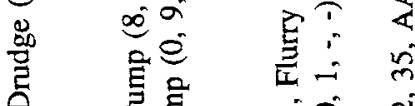

ह

0

ते 50

m $\dot{2}$

i

$\dot{\nabla}$

ái

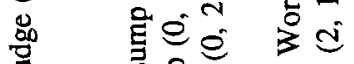

z

के

$+$

$\stackrel{8}{8}$

$\stackrel{\text { I }}{\Xi}$

㺃 i

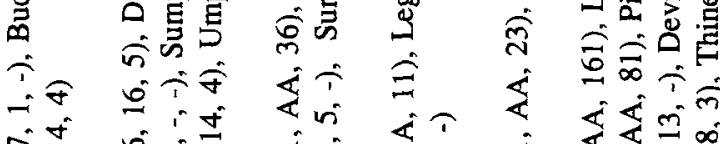

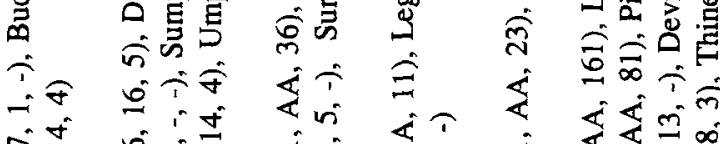

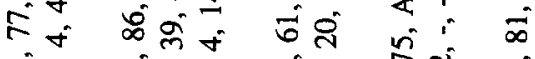

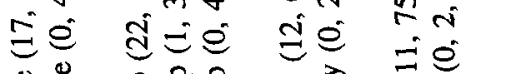

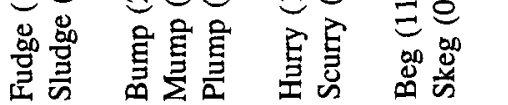

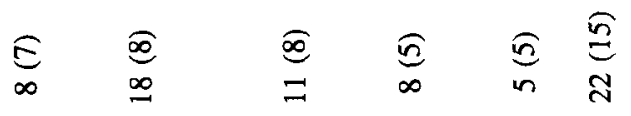

\&

부 $q$ q

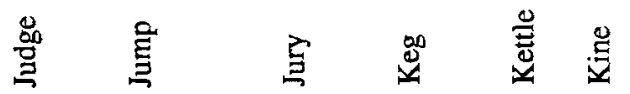

$\hat{\sigma}$

$2+1$
0

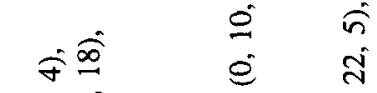

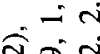

i

的言离

$=\dot{i} \sigma$

$\stackrel{\mathscr{G}}{10}$

ङे :

运葛.

तंक

$e^{\infty} \infty$

要至定

is $c$

安号节

棺宗

¿̇i

ถี่

aㄹ

运要

จำ

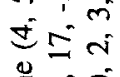

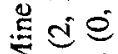

量黄

ते

यदे

सित 42

exid

苟造

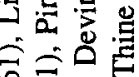

\&安向

ลำ

$\therefore= \pm$

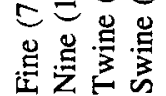

\section{(5)}

\&

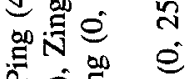

iิ

की $=\frac{1}{m}$

量是文

คิ⺈

于े

这它

के

$m=\infty$

驾能

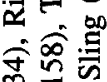

这定

कंते के

me

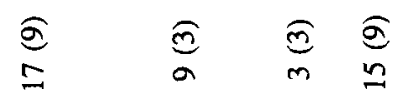

g

\& $\infty$

过参高芯 $\stackrel{\infty}{\infty}$

$\hat{n}$

$=\hat{i} \quad \stackrel{\infty}{\Xi} \dot{0}$

$\therefore \dot{0}$

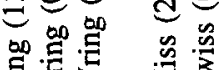

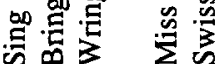

要

iे

ज范

这

in

承

它

वे

80

ś

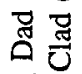

ते

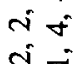

is

sic

m
ñ
ñ
ते
m
n.

乎

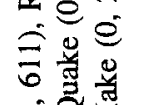

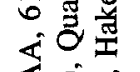

यंत

我延

常

定芦

m

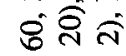

ปจิ

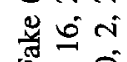

ब.

可 敢

穴

4

$\infty$

令兽

की

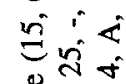

鬲

胥

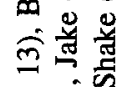

守

in

4
$\infty$
$\infty$

兽

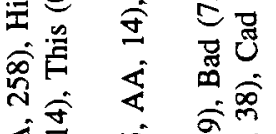

穴角

से

रूं

रूडe

षें

miñ

चुच

党

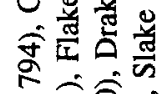

过守主

कीष्त

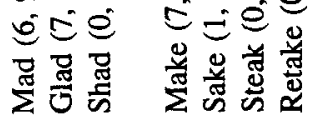

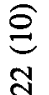

$\stackrel{\infty}{+}$

莽

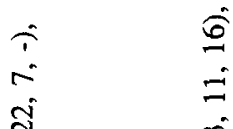

e $\quad \hat{=}$

है

iूल

तेंत यु

تீ $\quad$ iे

दें

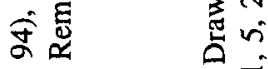

<六

守a

लत तू

远

केष 运安

i $\quad \dot{\text { gं }}$

ñ்

求学

表要

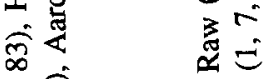

\&余完

ลंก तें

लंष एँ

远

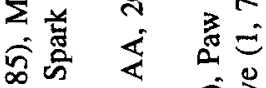

î

文

กั

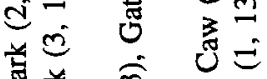

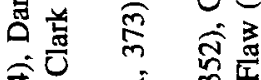

ब延

सं का

की $\approx$ के

过

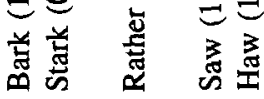

$\underset{m}{\infty} \underset{\sim}{\stackrel{\sigma}{\Xi}}$

용

盖慰言 


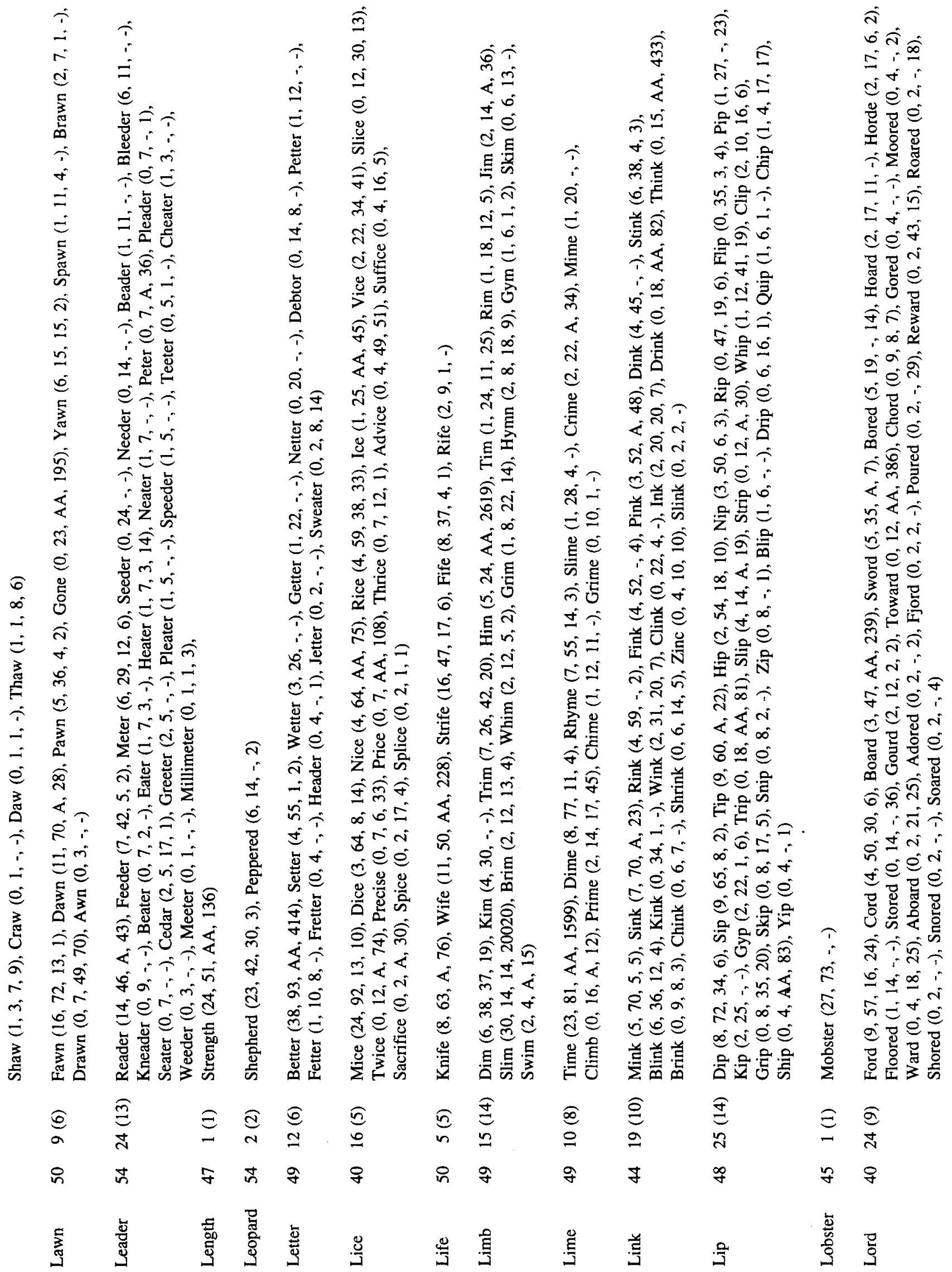




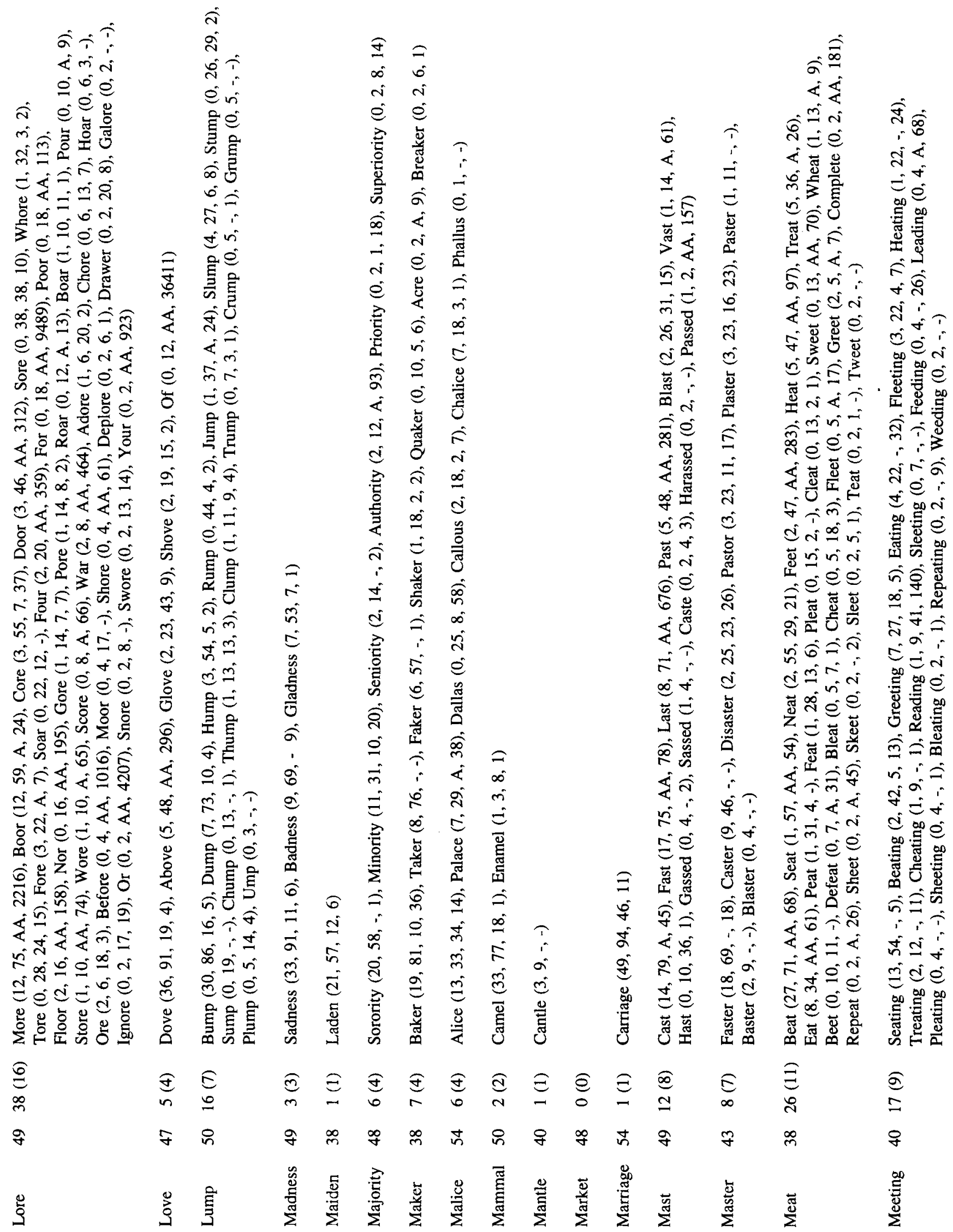




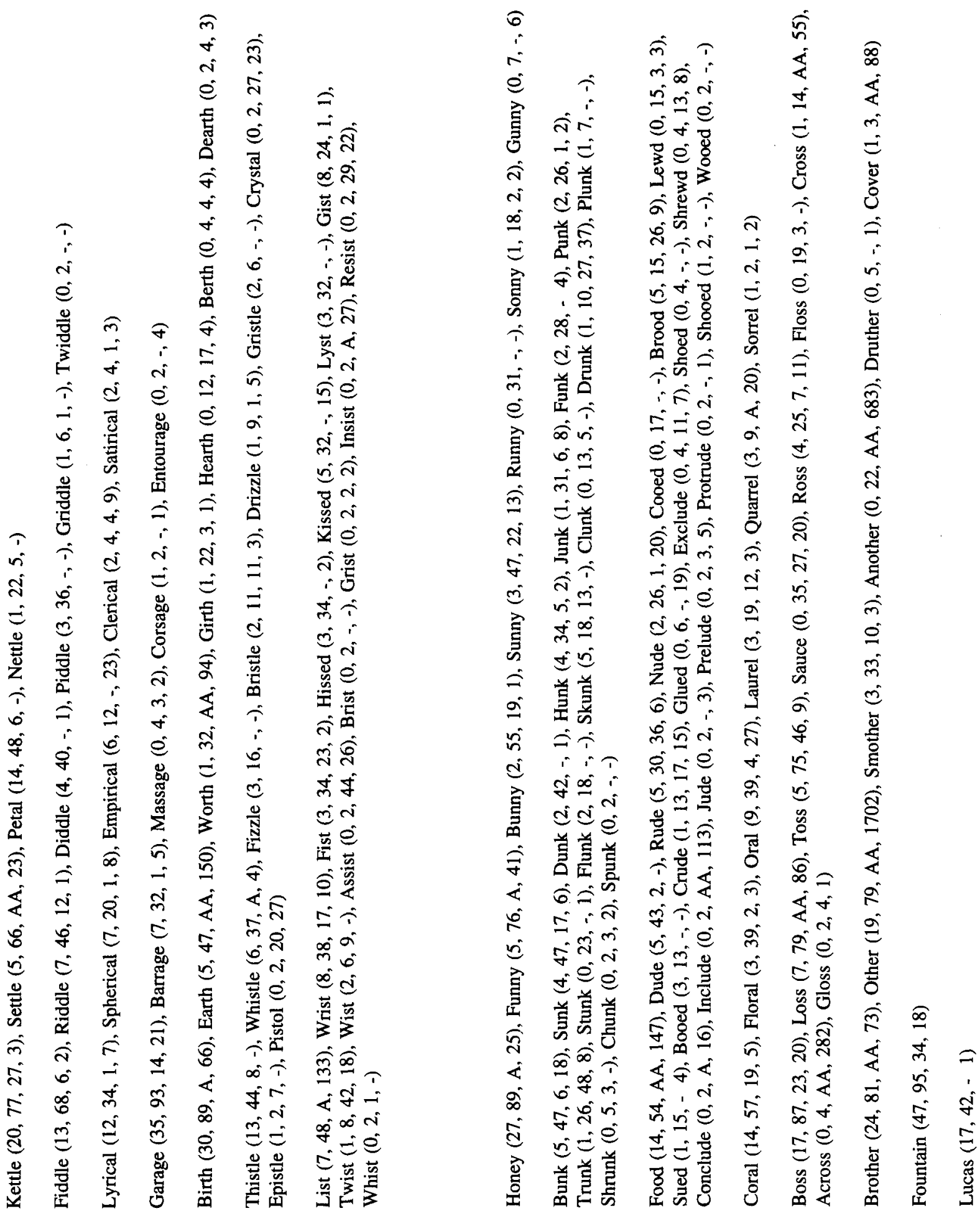

仓)

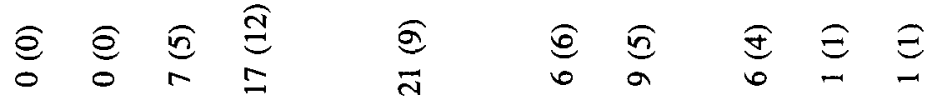

丹

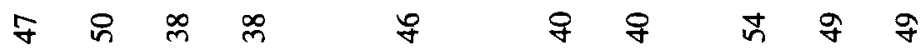

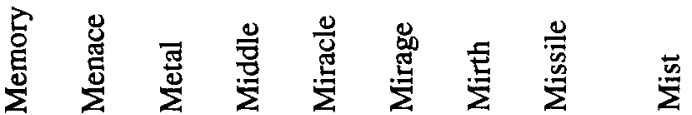

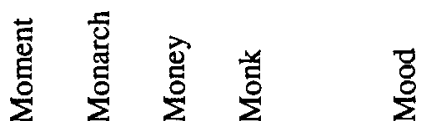

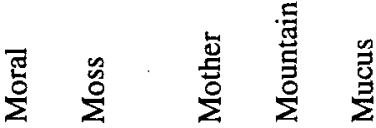




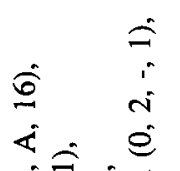

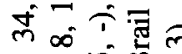

$\sum \underbrace{\circ}$ in

卷

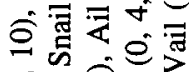

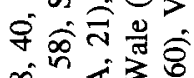

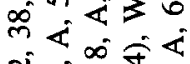

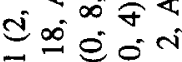

丞过过

蚛泀

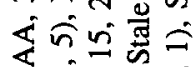

$\infty a^{\infty}+i$

$\stackrel{\infty}{\infty} e^{\infty} \dot{i}$

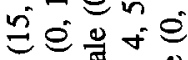

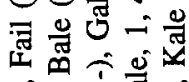

$\hat{\sigma} \hat{=} \infty \bar{\infty}$

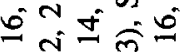

ทें

อ

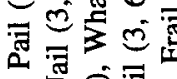

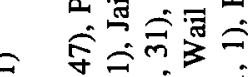

$\Rightarrow \quad \dot{m}<-$

$\therefore$ 的㐫五

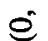

ळ

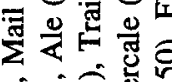

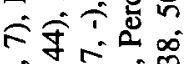

vi

in $\sin ^{\circ} \hat{e}$

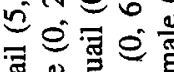

范苛产

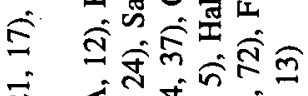

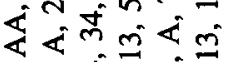
gी

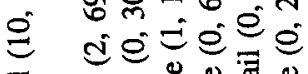

常

ส

의

量言

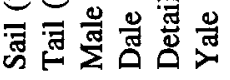

m $\quad$ i

จิ $-\dot{4}$

in

î

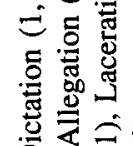

鱼完。

कิष

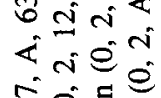

¿ंe.

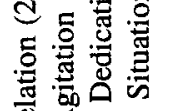

帚药穴穴

ลิโธิธ

त :

000
200

e.

도ㅇㅝㅠ.

可贻色

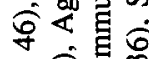

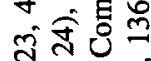

政安

din in

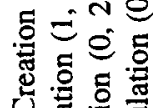

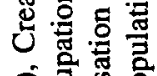

守気帚这

m.

$m$ 过

वंत्व

in

迅

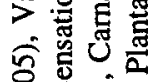

点宫

运的

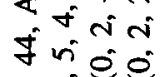

نंe

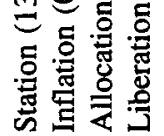

ळ

i

$\infty$

高

惫

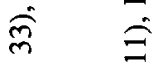

क के

$=\quad 5$

苟

ธิธิ응

in m

in

कิ

के

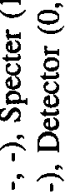

总

$\infty$

$\approx \pi$

$\simeq \quad$ 은

\&

$\infty$
$\dot{I}$

$\overrightarrow{0}+$

e

पे

$>$ 总

i बे

- त d

$\therefore \frac{1}{0}$ में

ते हैं

然

ن के

कि

चंत

जं क्ष

委 \&

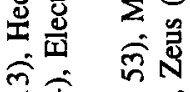

i. 4

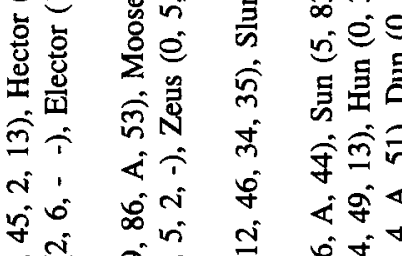

$\infty$ 过

包造

密总器

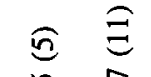

ร 9

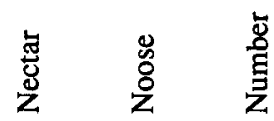

咅

妾

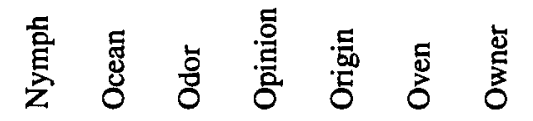

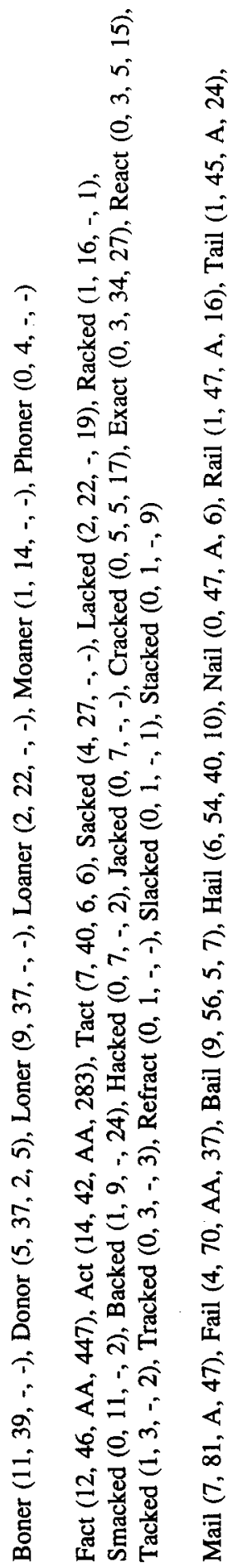

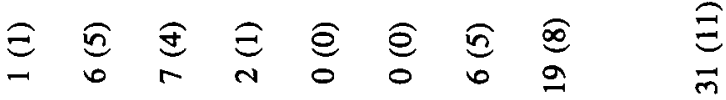

$\forall \forall$ \&

字

: 


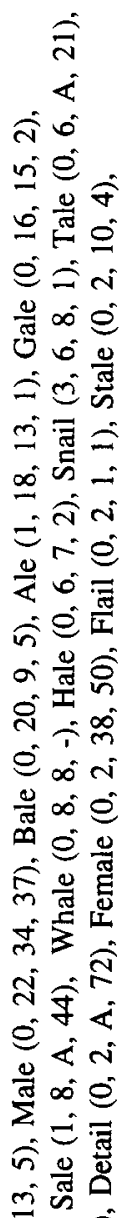

लंक

两in

$e_{\infty}^{\infty}$ in

ते

穴安霥

ิำ

สำ

लें

$=\bar{e} \hat{e}$

票需的苋

Iิ $-\hat{0}$

दin

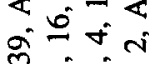

¿ीe

素高斏

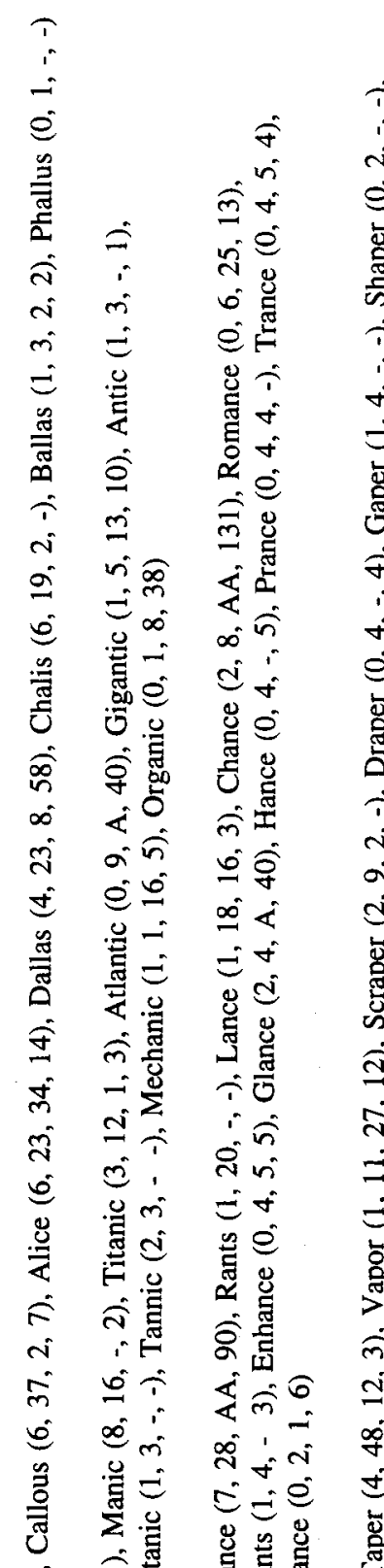

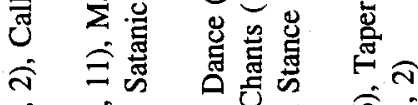

ㄷำ

की

ते ڤं

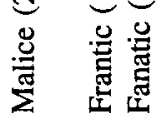

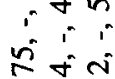

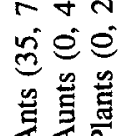

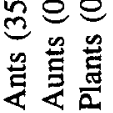

$\underset{0}{0} \underset{0}{\infty} \stackrel{\Xi}{\infty} \underset{\infty}{\infty}$

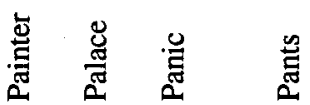

F $₹ \stackrel{\infty}{q} \underset{m}{\infty}$

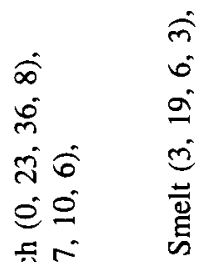

仓 $\quad$ हैं की

हैं

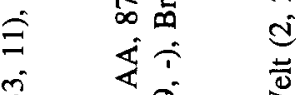

m एक एं

$=\quad$ के

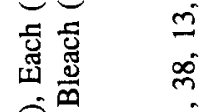

$i \quad \hat{i} \dot{0}$

i $\overline{8}$ व

7 $\quad \dot{0} \quad \hat{i}$

焉

în

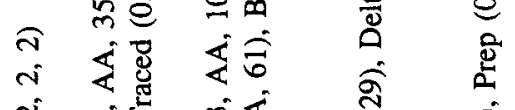

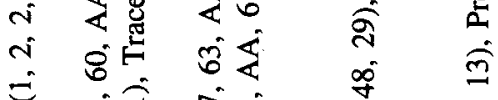

च 仓

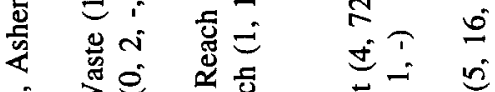

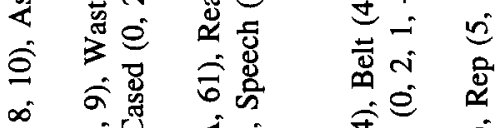

f $\infty$ की

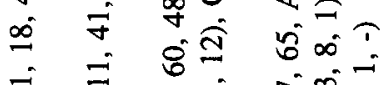

$\stackrel{i}{\sim} \stackrel{\infty}{\sim}$

$2-i$

i i a

चี $e^{\infty}$

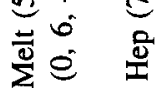

守总离

完弯

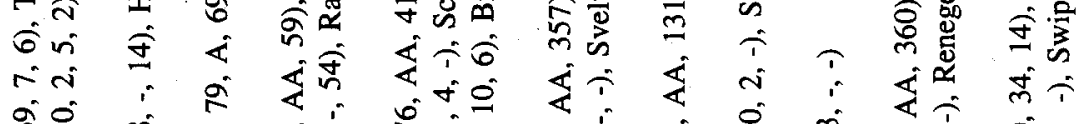

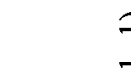

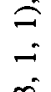

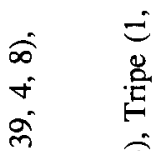

$=\quad \dot{\nabla}$

$\stackrel{\infty}{=} \quad=$

0

东产

i

$\stackrel{n}{i} \quad n$

i

f

Ti

จำ

ลิं ले

$6 e_{00}^{e}$

$\infty \overbrace{}^{\infty} \frac{\infty}{3}$

बิ

वें

ลิं में

m.

श口

을

宫芒

学些

จं

$0 ; 0$

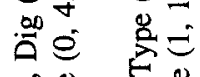

宫品 守芯

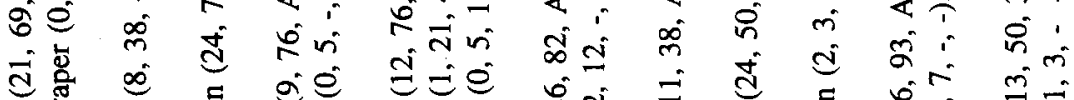

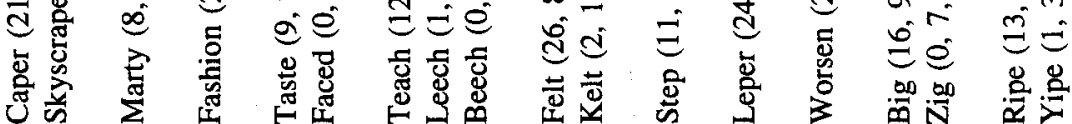

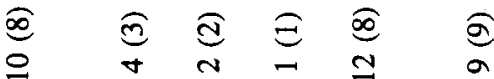

f 于

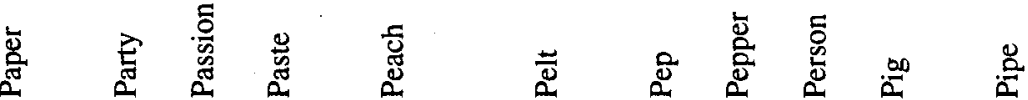




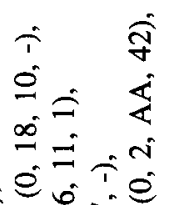

$\infty$ 过.

远考它

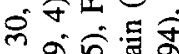

cक कं क्ष

ज割

- 0 i i

吉寻苞e

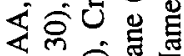

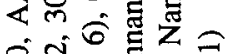

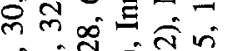

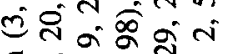

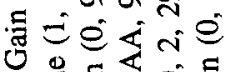

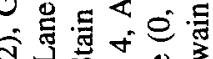
च क है จ

में

हैंण नंत व

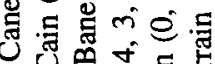

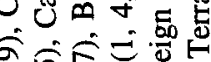
玉旅它 <㐫覃官的

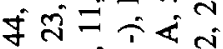
¿̇eñé

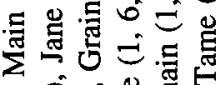

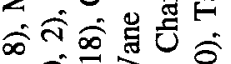

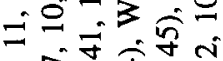
कें山ेंबे व 400 त

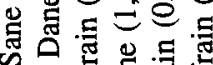

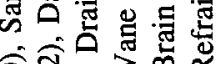

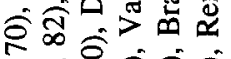
सं으료

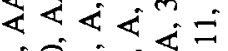
ơ écé巳

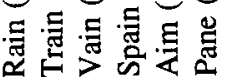
?<smiles>C1CCCCC1</smiles>

守-

这穴?

过这

ér

空过它

के

तेषi

กิำ

ध

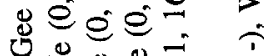

进这

तิ

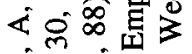

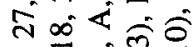

वंक

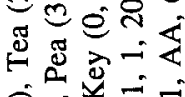

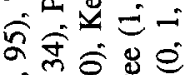

<我

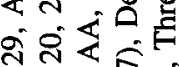

ceक

\& $8<8$

क

तิ

\&这市

$\ll<\infty$

กิ

$=\hat{e} \omega^{\circ}$

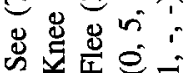

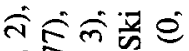

+हुन

बें

तुं ते

तथे nू

考 $e^{-}$

學嵒

穴密

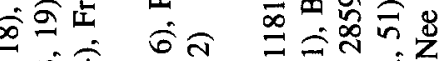

तें

तें

$\therefore=$

㮍总兽

$<0$

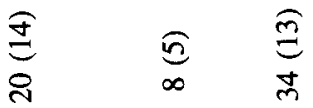

n क से

壱 蒙 di $=$ in

कीष

的导

$\therefore$ 定

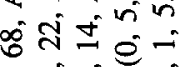
¿さं

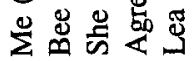

$\underset{\mathrm{m}}{\stackrel{3}{\Xi}}$ i ㅇำ iิ

¿

$\frac{8}{0}$

i

กิ

in

递

㟧

क

过

in

莡

กิ

ते

in

vi

造

衣言家

+人 क守

主

i

$\stackrel{0}{0}$

兽

हों

ñ $=$

형 $\overline{0}$

से mi

$\div$ i

๑ే

ลิธ

तें

जं कि

它。

离官

सें

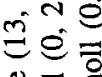

종 종

守它幖

\&은

กீำ m

ก

(

c

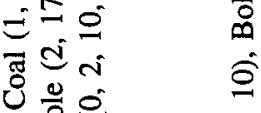

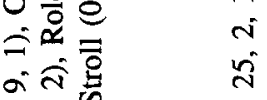

वंचक त

तैंิ ते में

c $=$

훙 웡

के

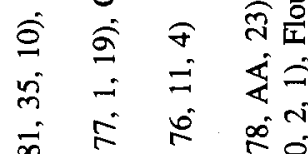

$\infty \div \infty 0$

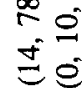

它 $\therefore \quad \dot{\pi} \quad \dot{a}$

$\exists \quad \therefore i$

$\infty \quad \hat{\infty} e^{\infty} \hat{e}$

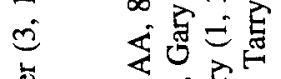

莒

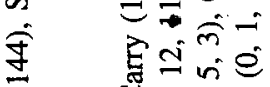

\& Uंm

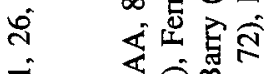

$=$ काल

气 लेखि

I.

i $\quad$ in

\%

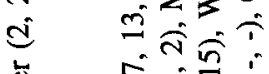

峁

¿

nं

$\because$ की

बi 0 क⿺辶一

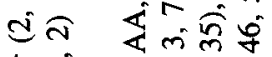

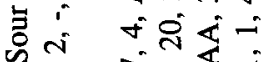

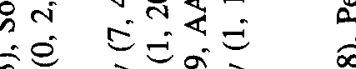

กิ

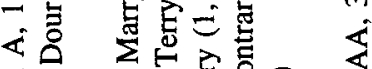

एक कें

एं कें

प0 की

है $000=1$

च

昰它志

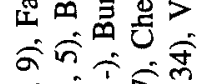

in: $\infty<$

civ $=-i$

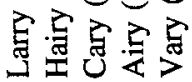

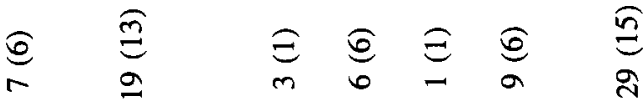

ล)

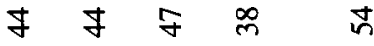

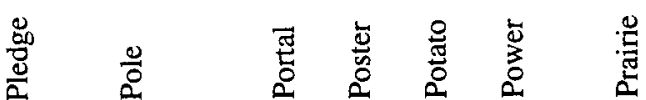

อ

㒕

$\approx$

i

镸

$\therefore \quad \frac{1}{2}$

$\stackrel{m}{=}$

蛋

畩

N

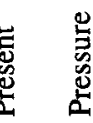

弯

ก 


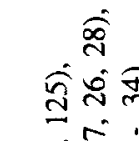

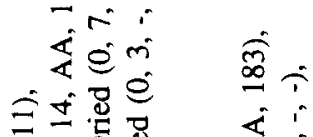

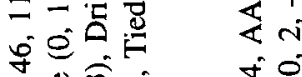

鸟 $\hat{i} \quad \dot{0}$

ज政

的氙

子势

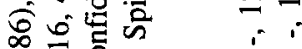

$\therefore$ 过 0ंत

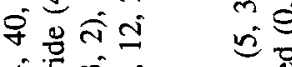

एٔ

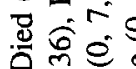

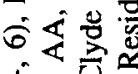

6ं

o $=0$

g.

वंकेष

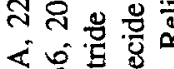

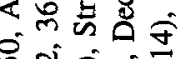

की

$c=\sigma^{\circ}-4$

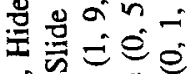

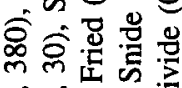

\& $4=$

in

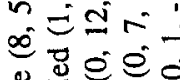

果

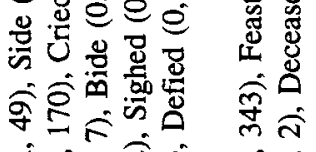

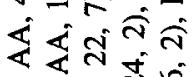

वत्त式

बंब这

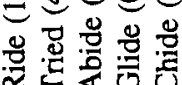

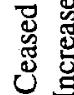

तิ

<i:

$\infty$

s

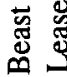

के

\%

我

政

官官

if $\infty$

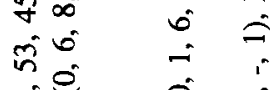

क⿺辶一

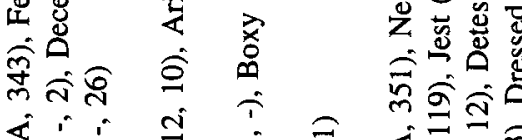

एक

$6+8$

c

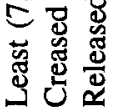

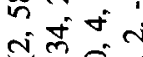

离 $\therefore$.

$\forall-i e$

$\varepsilon \hat{\varepsilon} \dot{\theta}$

边的

$\therefore$ 过

की

苟

穴

$<$ 政

ब․

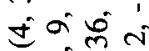

जै

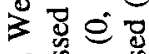

के

唡:

वर्ष

प्ष

के

要哥产要

खिं

मीं

की

过式

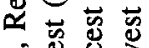

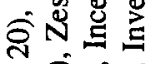

$4=0$

of

过

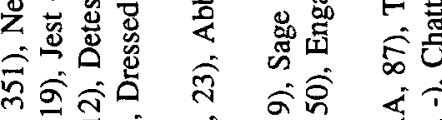

ลิ

ล

ทे

is

ie

0

畨

है

$\dot{i} ;$

क्ष 4

कूत क

शिंत

छेê

○ी

कें

$\widehat{\sim}$

is

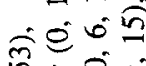

包

延出

तेक

mं

过定

角过

â

mi

जा

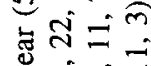

Ė

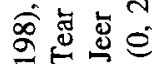

运完兽

于发通

vi

造

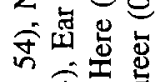

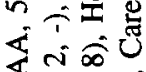

ज्ञ

in $\begin{aligned} & \text { in } \\ & \text { in }\end{aligned}$

这它

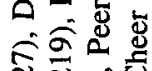

ปิ

这完

4 过

0

5 두

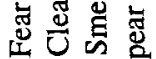

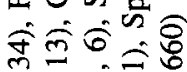

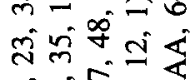

: $=0$

ôe

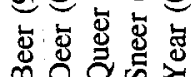

$\begin{array}{ll}E & \widehat{a} \\ \sigma & \infty \\ q & q\end{array}$

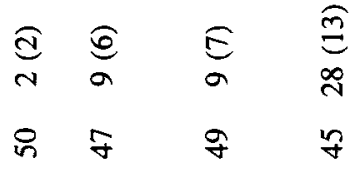

萑 $q$ 共 $q$

$\stackrel{0}{\Xi} \quad 0 \quad \sqrt{0}$

f

F

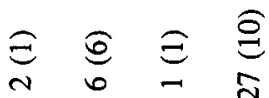

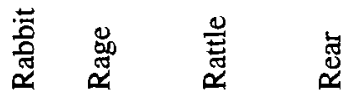

ㅇ $q$ q

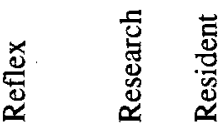




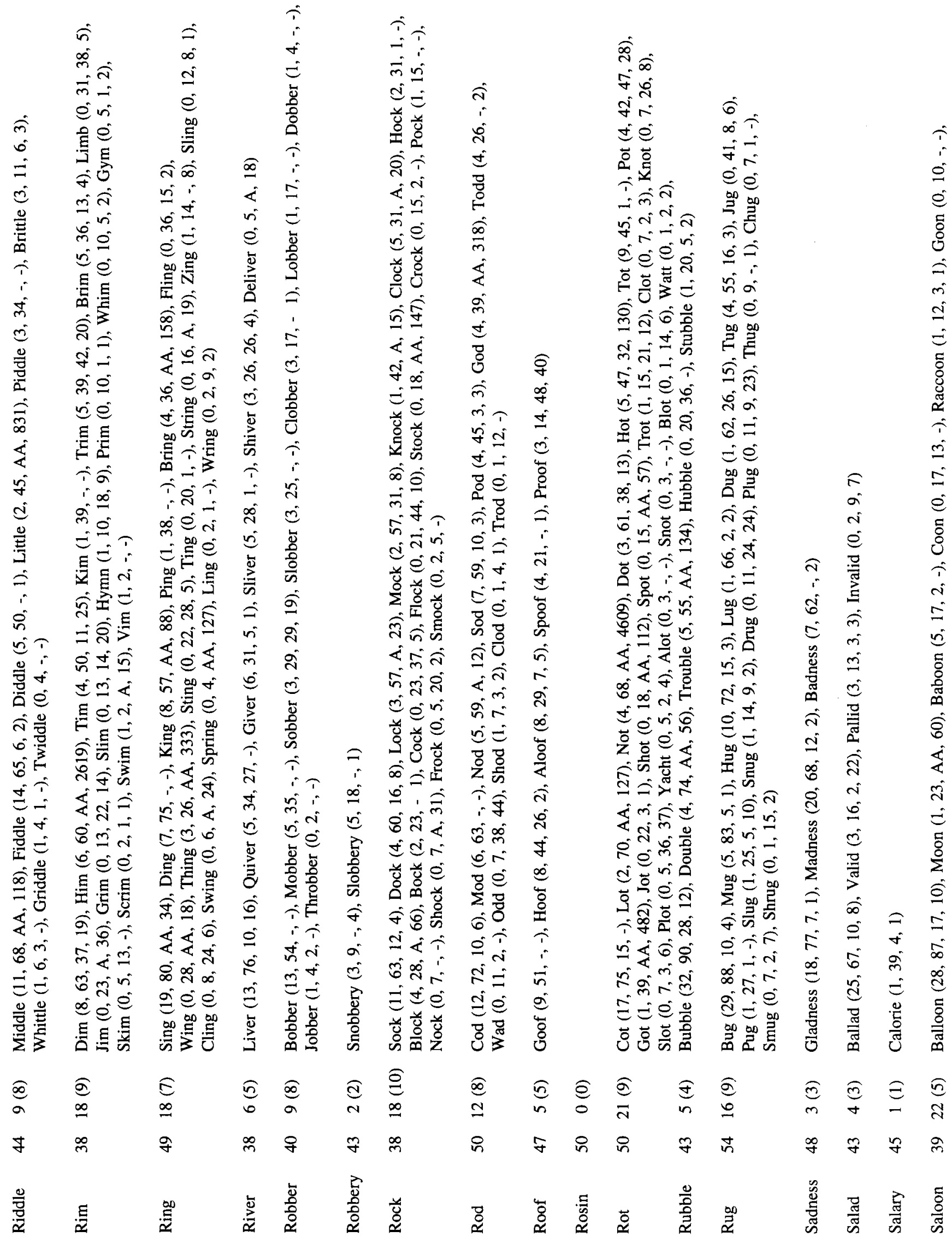




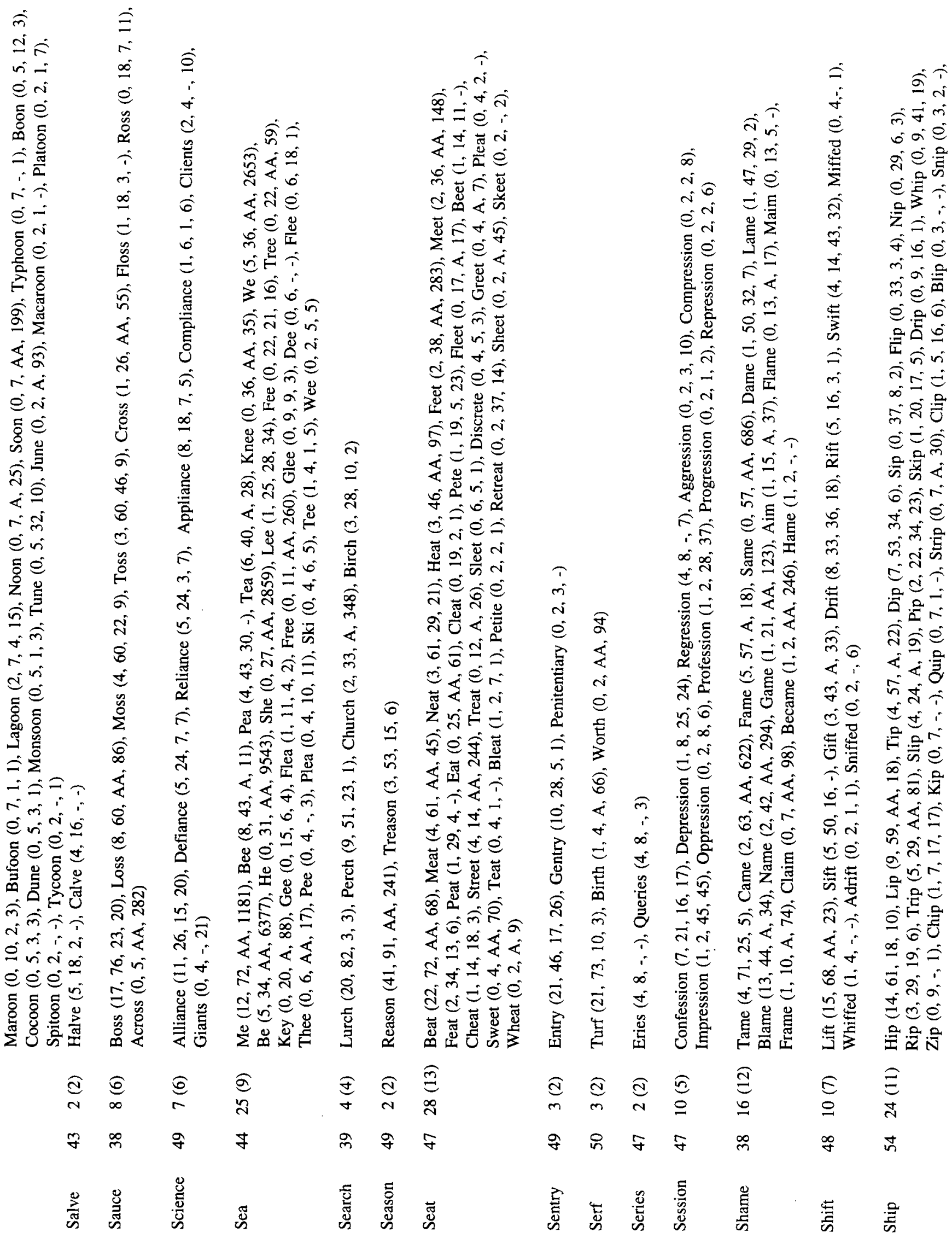




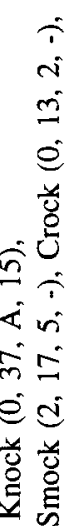

กิ

戈

बi

¿̇

䓀

$\dot{\sigma} \dot{n}$

ㄷํ

के

घं

卷

की

过

कों

过e

苋 首

응 过苍 तิ

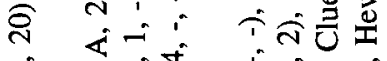

in

$\therefore b m a$

e 5 पे

是过察 के

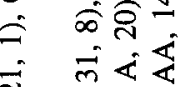

$\stackrel{0}{-} \dot{m}$

e vidé

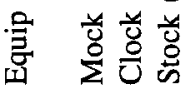

$<00 \hat{0}$

的焗密

의

w.

岂方向

过

สิ่

iो

तथ

ơ

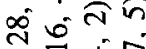

vi $=0$ in

: 0

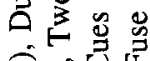

त

वें

ca

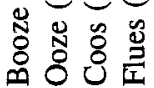

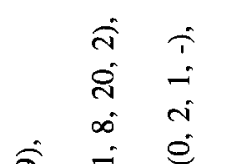

离范

造总苍命

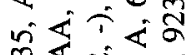

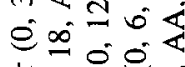

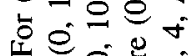

ลิ

m的垔

वंति 6

vi $+00 \mathrm{~m}$

过

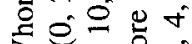

造它

穴重宛

م守出安客

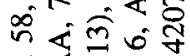

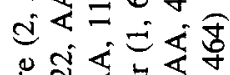

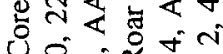

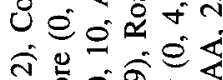

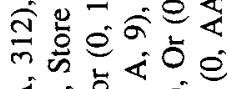

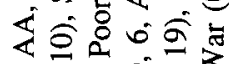

80 0 过

ชิ่

造这它

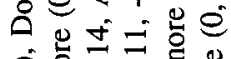

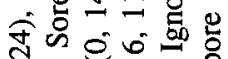

<合昰的

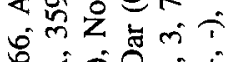

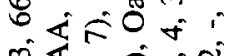

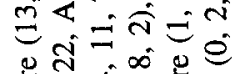

ปั

के

की

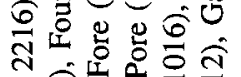

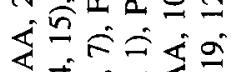

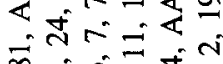

किले

¿

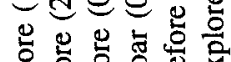

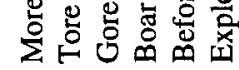

$\underset{\Xi}{\Xi}$

으

$\vartheta$

$\frac{\check{8}}{5}$

F

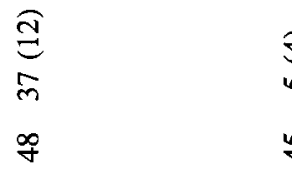

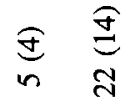

$\dot{0} \dot{0}$

तิ

过

नें

的㮍

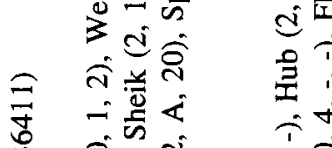

तิ่

8

e

o

a

$\stackrel{9}{9}$

co

8

ì

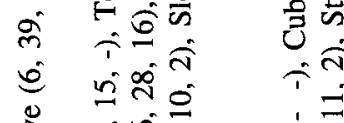

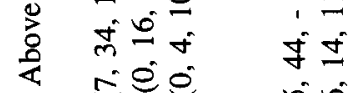

$\hat{\nabla}$

a

的鬲忌

तิ $\hat{\sigma}$ б

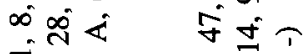

तो $0+$

ले

ค่ं

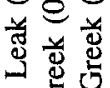

它它官

$\hat{0}_{0}^{\infty} e^{n}$

蛋总春

in $\dot{0}$

ina ố

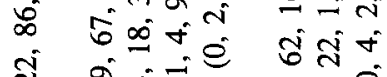

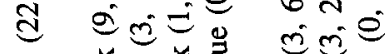

旁旁衰高

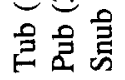

ๆ

छ $\quad \stackrel{0}{\Xi}$

总 岳

สิ

守 $\dot{n} \dot{4}$

$<=0$

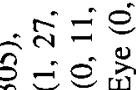

这的官

कें

我:

效安

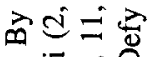

闹

तेंह

$\leq 0$

सेंश्र

viét

$\because \underset{\alpha}{0}$

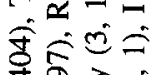

守层

这安市

के कें के

eत्य

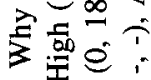

穴穴客。

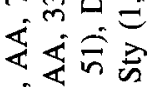

किषे

ปู่ส

文e

到含芯e

延余定

बिंदें

cी

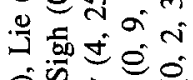

कें

स⿺辶े के

ปें

व $=\dot{=}$

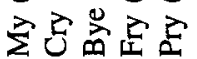

$\stackrel{6}{0}$
$\cdots$
7

竎
悬

空

우

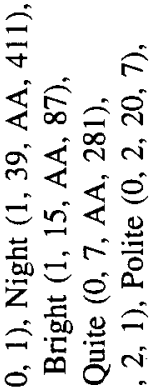

$\therefore$

ति

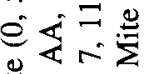

过这

مᄋ

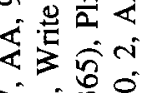

in

过这需

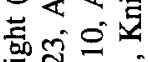

मิ่

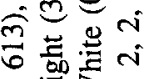

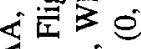

¿ิ่ง

8ू बू.

过余

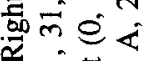

式营

ले

廷昰菂

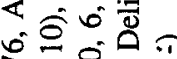

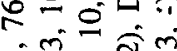

mं

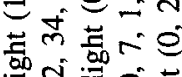

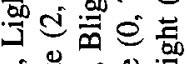

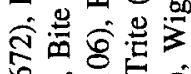

迹的市

य क m $n$

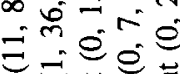

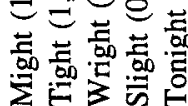

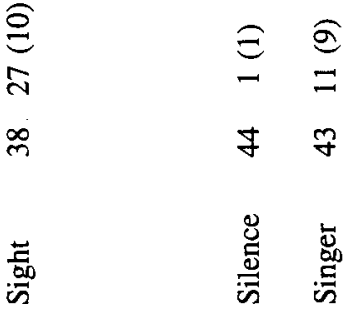


$+\frac{10}{20}$

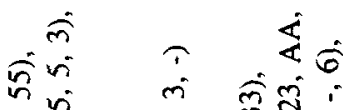

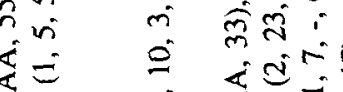

मे हो

言

政

3

o 5 क

手言

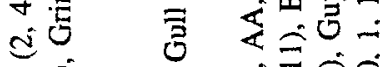

토

क대

के के

ल क

F 言

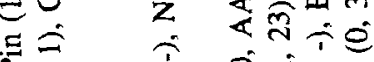

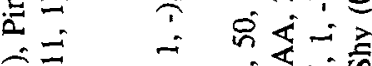

$\alpha=0$

$\because$ के

可

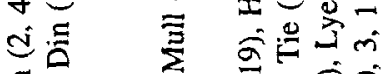

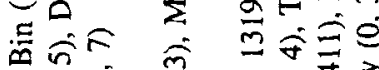

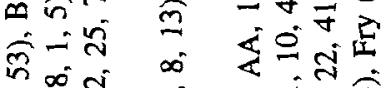

$<$ 造

in

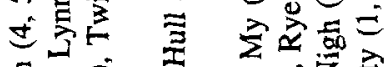

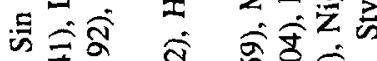

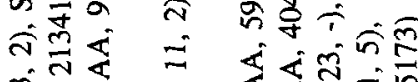

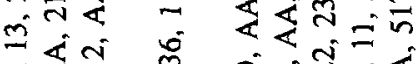

कीय के की

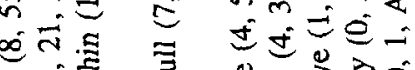

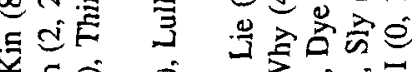

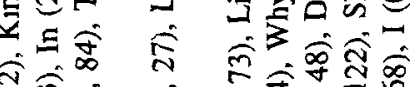

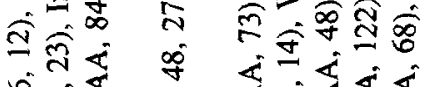

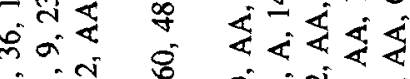

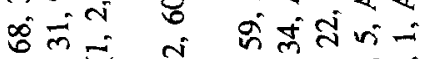

se.

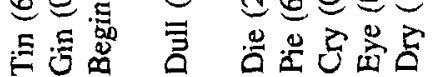

ลंक

तi

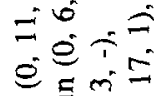

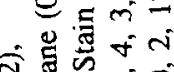

o ô कि

过军更品

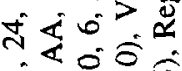

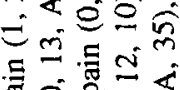

है हैं⿰彳

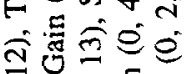

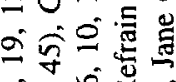

लष्ष

=约

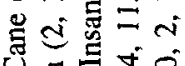

U.

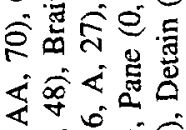

的全官全

वर्त诲

न 5 is 4

च $\hat{0} 0$

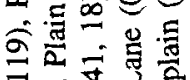

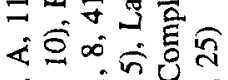

करटंजे

स्व हैंत

है

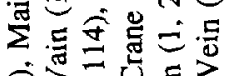

के

को

$f=z$ iो

कत्त की

ज承

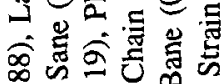

$<$ ते $=0$

$<\div 0 \div$ 迹

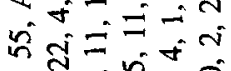

$n^{-1}=0$

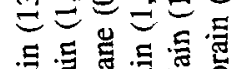

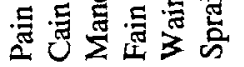

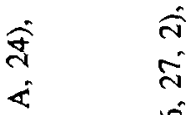

8) की

a)

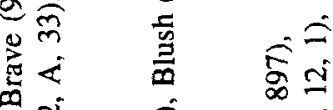

ลิ

ज $\quad$ त

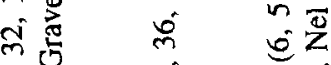

=ं क

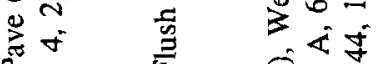

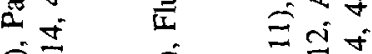

$\hat{\sigma}=\hat{\sigma}$

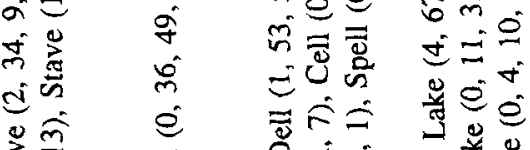

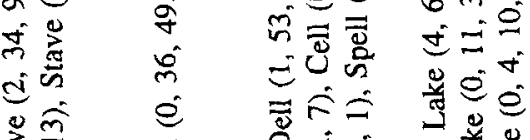

न क्ष

$\therefore$ 제

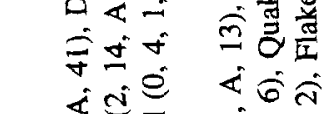

-

年

赵

可

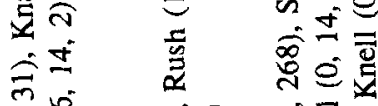

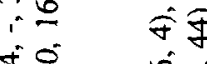

i

焉 $\quad+$

बิ

बิ广

यक कि

पै

vi

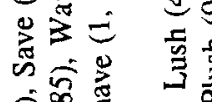

ลำ

iti $\hat{i}=$

$\therefore=10$

छब仓

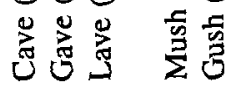

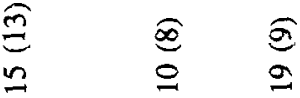

g

商

मीं स्त

diิ

过

票家

\&ण

को

$\because 0$ ¿

छें

च $\overline{\mathrm{c}}$

尊

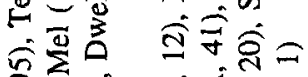

के संत्र

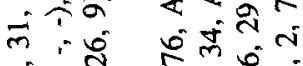

जि

in $e^{0}=$

$\dot{\Xi} \doteq$

证

च

की

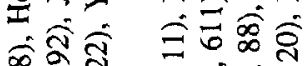

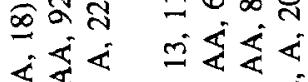

840

वंज 0 $\infty e^{-0}$

$\infty$ is

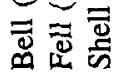

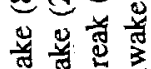

ส

$\infty$ q

F

獣高

岂

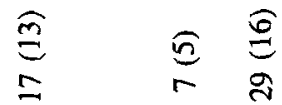

胥

言器言

2

i

$\hat{\varrho}$

응

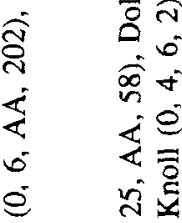

êt

突

की

सं

<官

$=\infty$

节字

뭉

S응

Qरत

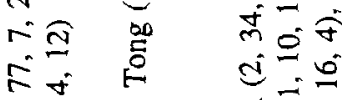

वर्व ब्ञ

ज्ञ

6 र

可 $\vec{\forall}$ 《र

$\therefore$ क

:

年

\%

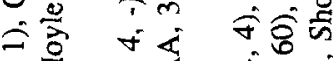

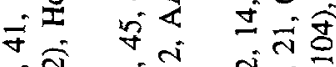

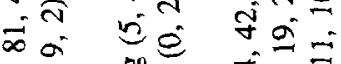

齐

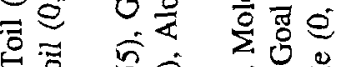

के 命

से 玄这

की क⿺

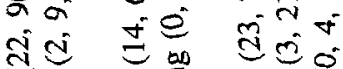

त्d

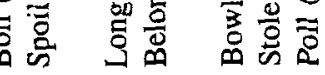

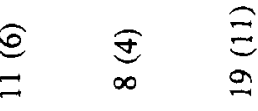

‡ 


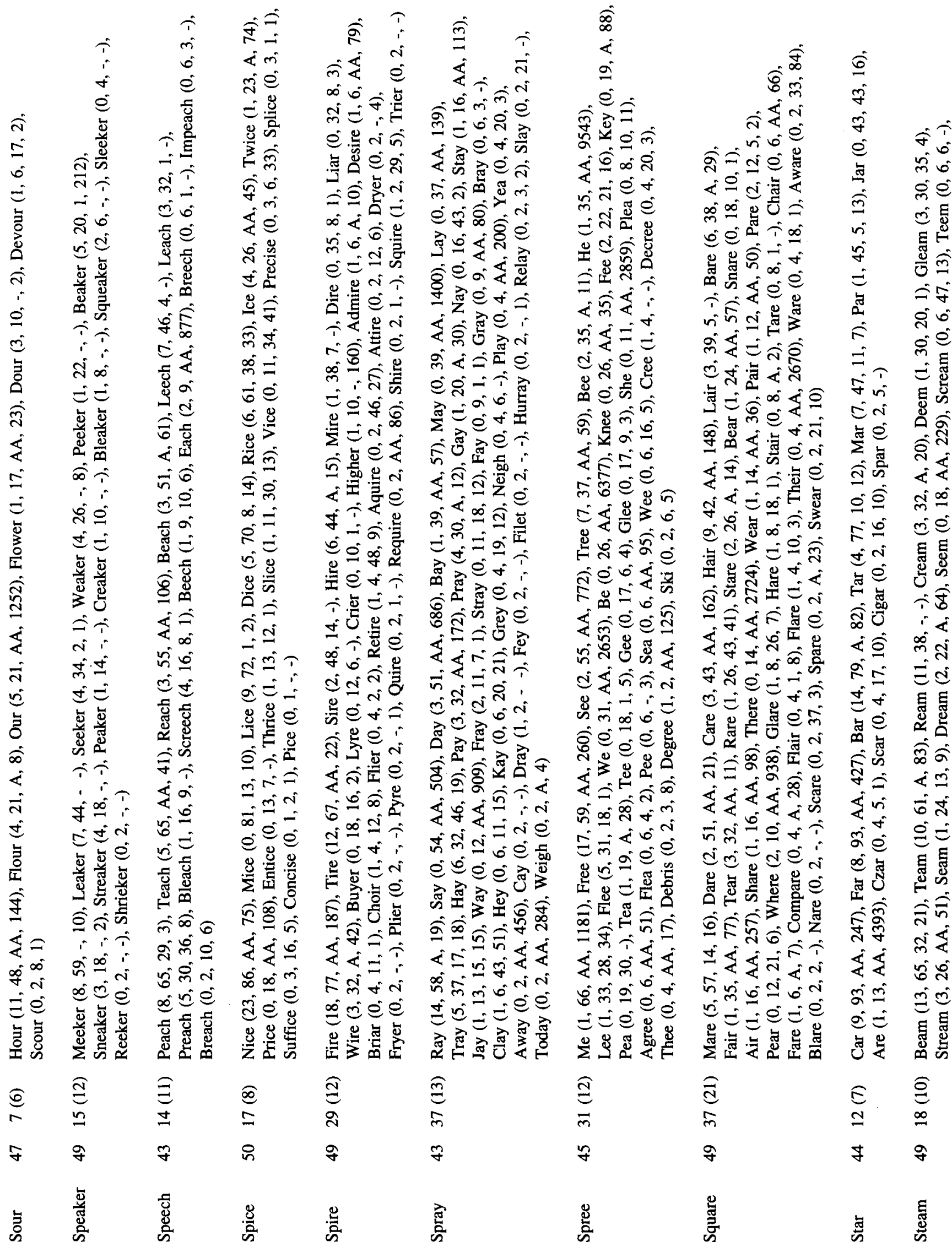




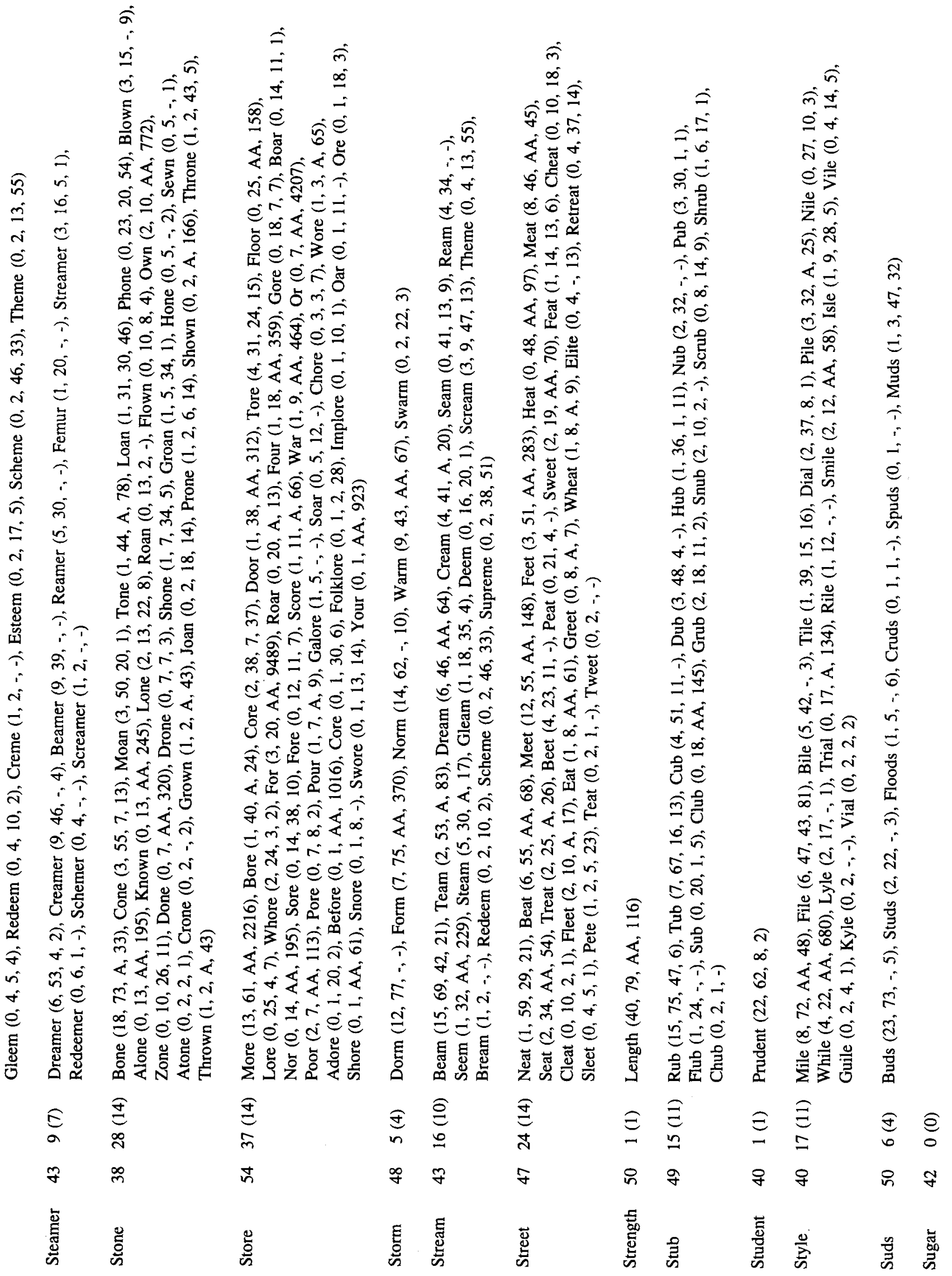


$\dot{i}$

i i

$\bar{e}=$

总薦

官 $\quad \dot{0} \hat{\infty}$

ले $e^{n}$ in

m $e$

$\Xi \quad$ 嵌

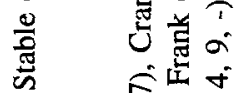

o $\quad \therefore \dot{0}$

त.

$=$ \& की के

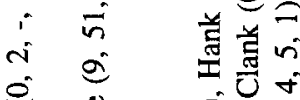

है है

ठำ

ㄷ

$\therefore$ i $\quad$ 过

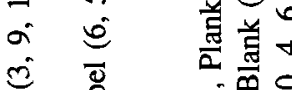

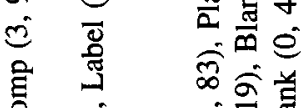

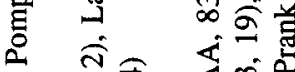

i

$\therefore \infty \bar{m}$ हो

$\therefore$ in

i $e_{0}^{\infty}$ 光

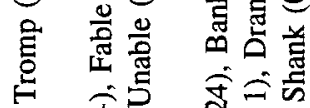

๘ ทं่

$\therefore$ के हैं

กิ ปீE

๑ बं

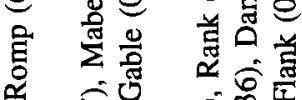

* हi

$i$ 穴

ส

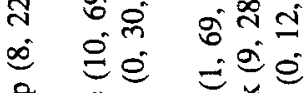

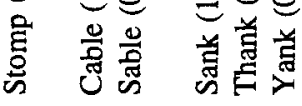

ล $\stackrel{0}{0}$ is

$\therefore$ ज की

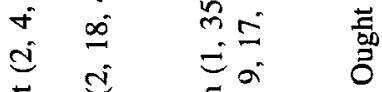

范

造 $\quad$ 运

旅安

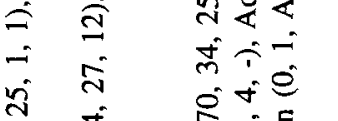

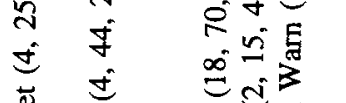

竞

焉 ह

के $\hat{\Xi}$ लें

+

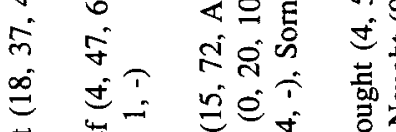

षे $\hat{0}$ 安

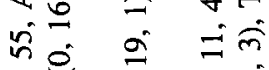

ลิ่

$\dot{\hat{\sigma}}$

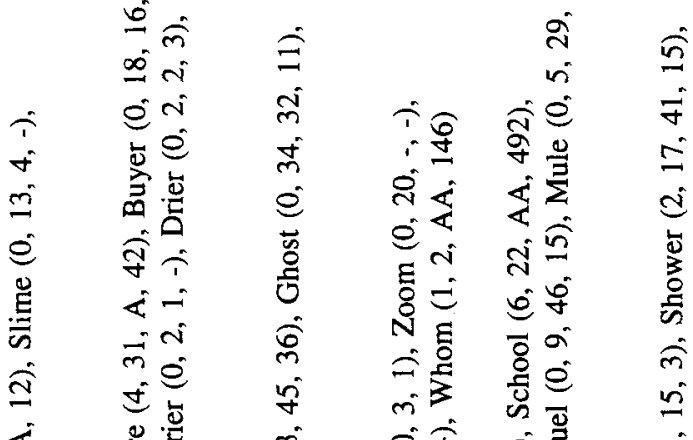

造出 E

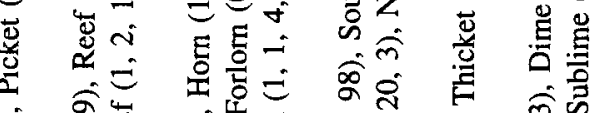

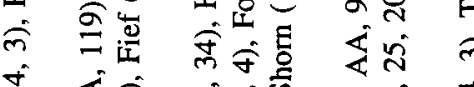

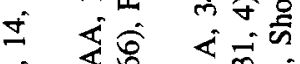

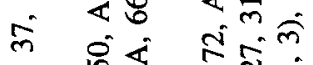

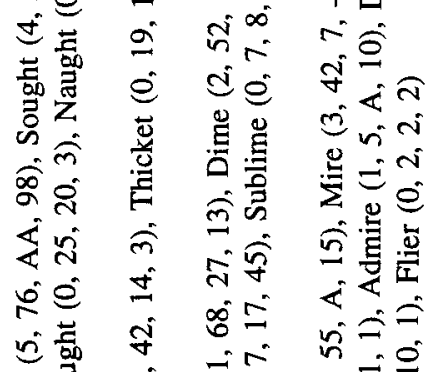

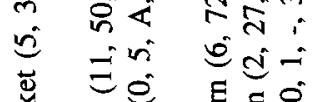

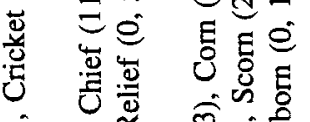

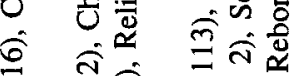

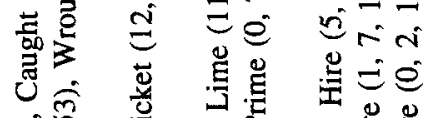

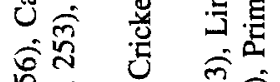

穴氙.

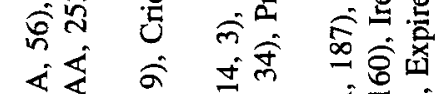

\& तें

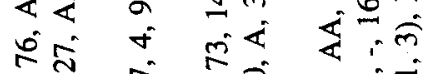

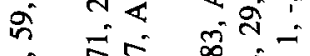

in 50

i $\infty \hat{c}$

0

$\begin{array}{ll}i m & m \\ \therefore \approx & 0\end{array}$

蔃茄

(c)

$\infty<$ कां

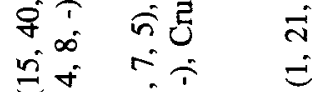

总总壱

至要

岛 过造

î

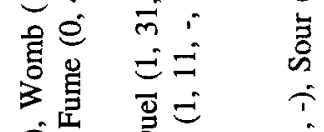

ลิธิ์

की

두 $=$

ชั

¿ $=$ बं

ह है

Ð $\widehat{\Xi}$

仓

in a तิ

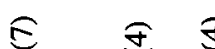

塎察

氙

$\overline{8} \cong$

क्ज क

\& $\mathrm{m} g$

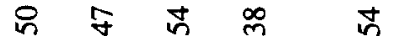

으

$\widehat{9}$

ङ $\Xi$

e

$+\infty \infty$

g $\quad$

\& $\quad$ i

$\vec{\nabla}$

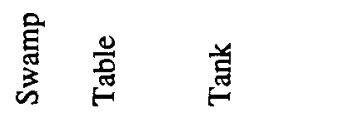

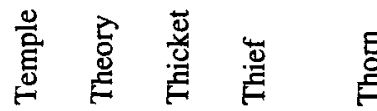

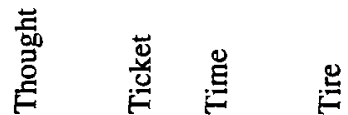

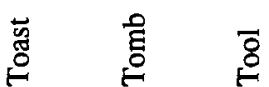

离 


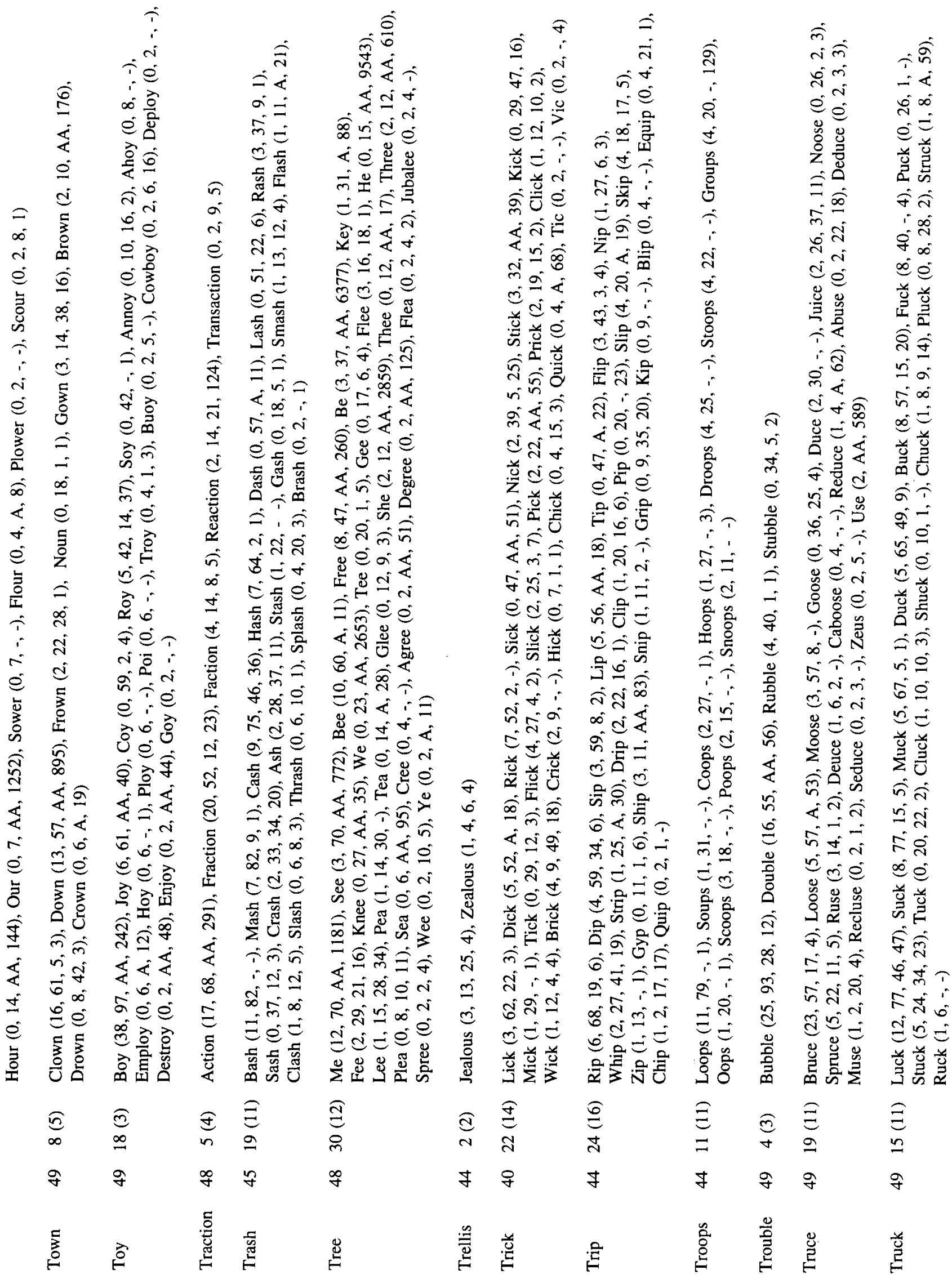


ti

i $\dot{\infty}$

$\approx \quad 0$

壱 $\quad \begin{aligned} & \text { E } \\ & \text { E }\end{aligned}$

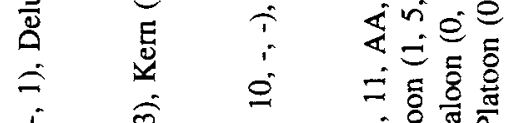

$\therefore \quad$ ते $\quad \therefore$ तो

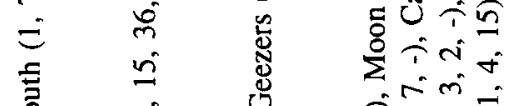

¿ $\quad \dot{0}$ i

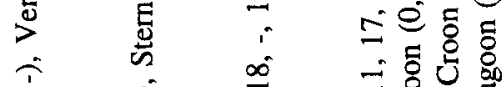

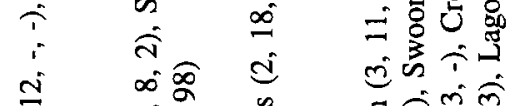

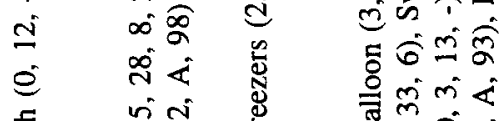

致

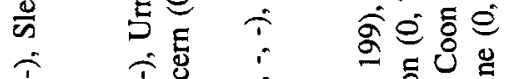

$\therefore$ +ं कं

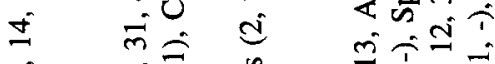

$=$ 过 总

吾 $\quad$ ह

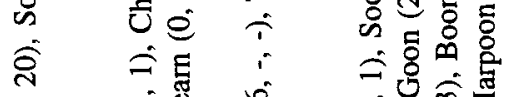

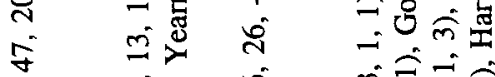

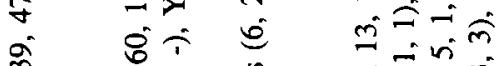

$=\quad$ ci

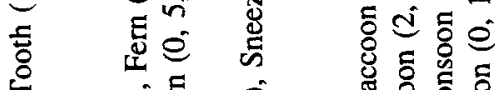

กิ่ के

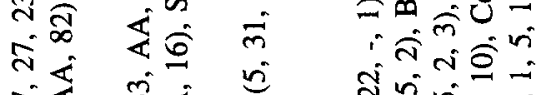

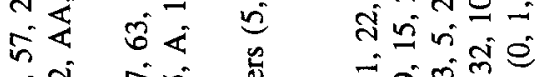

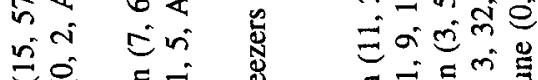

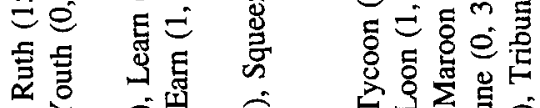

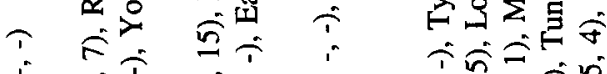

$\therefore$ जi

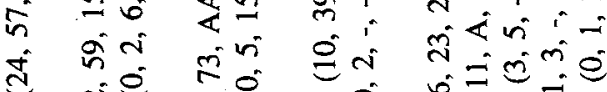

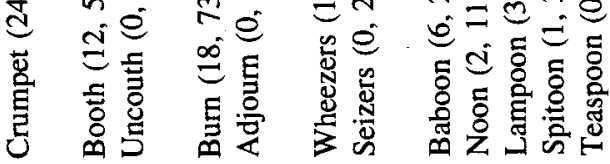

三

- a I

q $\& \quad \infty \quad \infty \quad$ i

In
6.

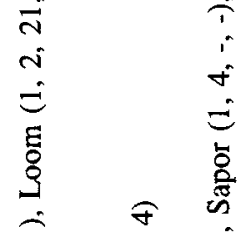

$\widehat{\Xi} \infty \hat{i}$

ते $=\dot{0}$

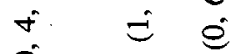

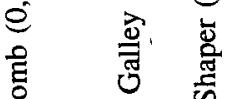

$\stackrel{\infty}{\infty} \dot{\sigma}$

$\hat{\widehat{m}} \dot{m}$.

\& m

ह 离

के $\rightarrow \infty$

के है

ث $\stackrel{0}{=}$

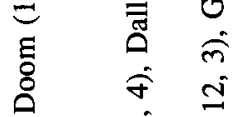

के वे के

\& $\stackrel{0}{0}$

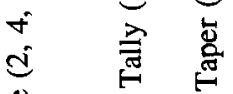

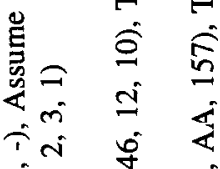

$\therefore=$ श

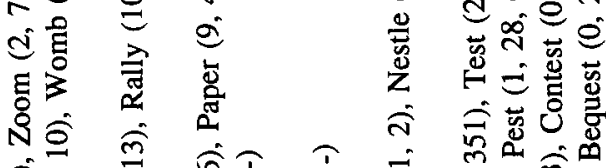

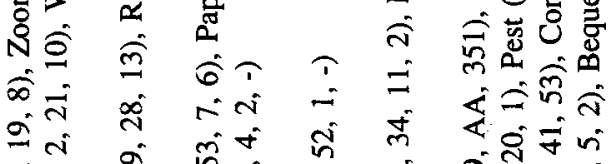

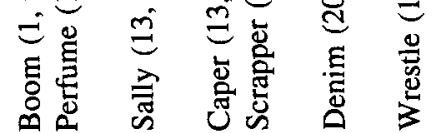
过过

जे

六路

సิ仓过

延苞宫

可:

6。ำ

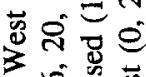

<范灾灾

में

溇

$\approx \infty$

O

व $\quad 00$

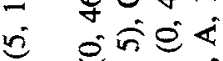

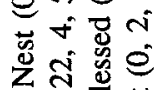

केलि口

菏客点

सेंक

तेंक
¿ $\quad$ छ

\& $\stackrel{i}{i}$

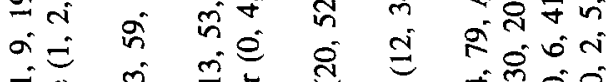

$\stackrel{2}{2}$

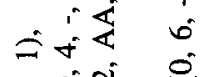

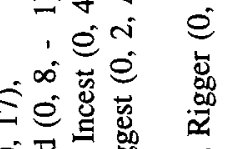

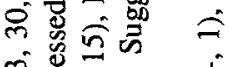

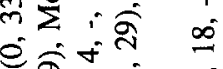

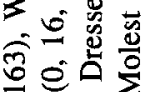

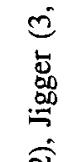

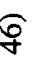

$\infty$

i

5

营

ह $e^{\infty}$

$\therefore$ 苛

ลิ $\quad \tau \stackrel{ \pm}{=}$

वे $\quad \dot{0}$ :

a $a$

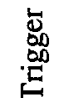

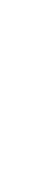

产

$\stackrel{a}{\Xi}$

$i \quad i$

is

i

离

กิ $\hat{m} \div$ ज

कं: को

过苞

总总

实的

홍

$\therefore i \quad i \quad \hat{n}$

तi $\vec{i}$ i

¿อ बैं

峁 总

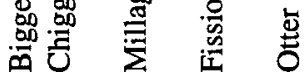

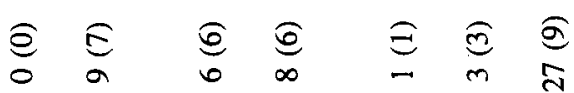

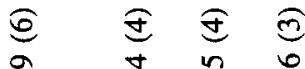

$f \quad q \quad q \quad \infty \quad \forall, q$

尔 g $尹$ i

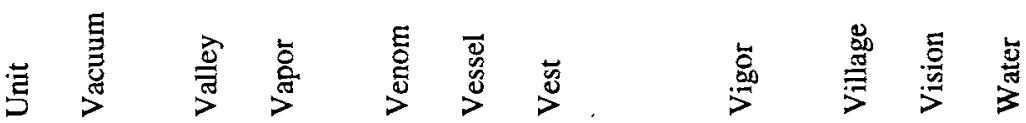




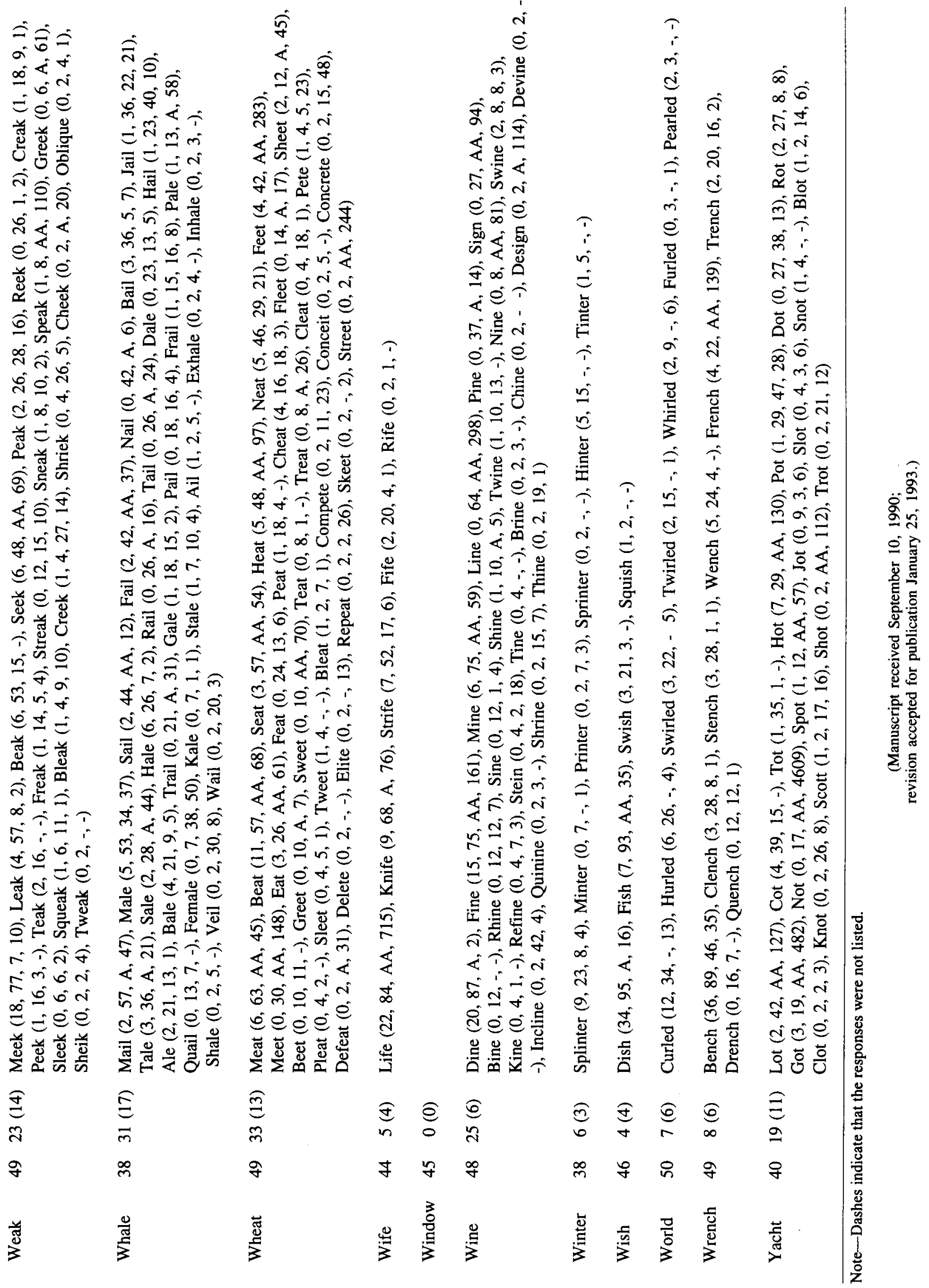

